\title{
Studies on Chicken \\ Hatchability and Its Relation \\ with Egg Yolk Metabolites
}

\author{
Dissertation \\ to obtain the Ph. D. degree \\ in the International Ph. D. Program for Agricultural Sciences in Goettingen (IPAG) \\ at the Faculty of Agricultural Sciences, \\ Georg-August-University Göttingen, Germany
}

presented by

Yi Zhang

born on 09.16.1988 in China

Göttingen, December 2016 
1. Supervisor: Prof. Dr. Henner Simianer

2. Co-supervisor: Prof. Dr. Christoph Knorr

Date of dissertation: 27.01.2017 


\section{TABLE OF CONTENTS}

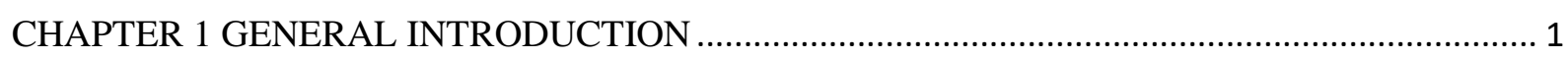

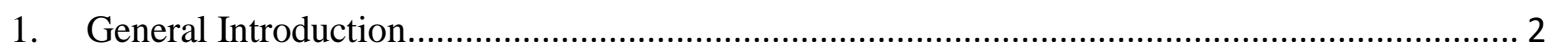

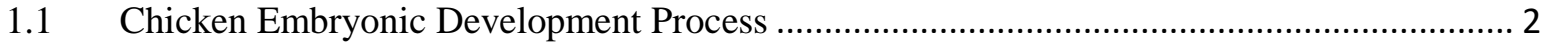

1.2 Factors Affecting Chicken Embryonic Development …................................................... 4

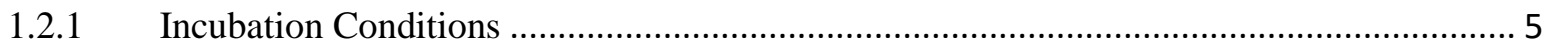

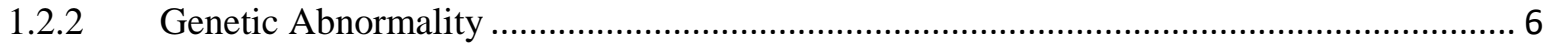

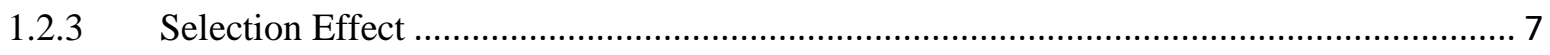

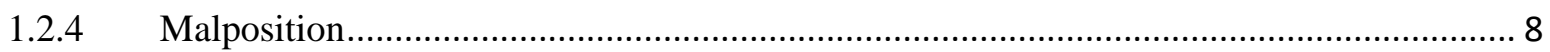

1.2.5 Chicken Egg Yolk Metabolites and Embryonic Nutrition .............................................. 9

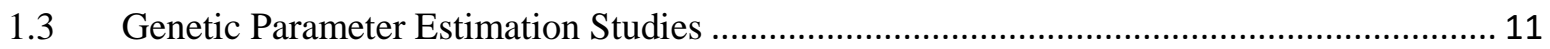

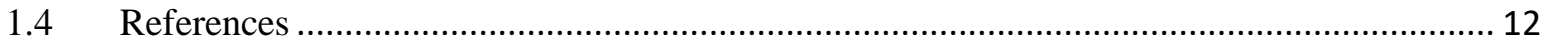

CHAPTER 2 METABOLITES IN EGG YOLK IMPORTANT FOR POULTRY DEVELOPMENT

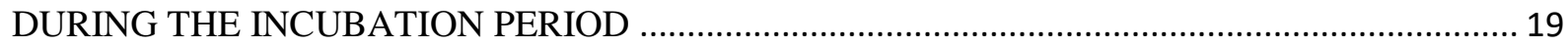

2. Metabolites in Egg Yolk Important for Poultry Development during the Incubation Period ... 20

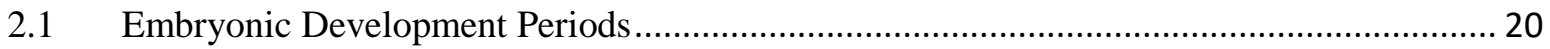

2.2 Metabolic Importance of Fatty Acids in Developing Embryos........................................... 20

2.3 Metabolic Importance of Carbohydrates in Developing Embryos ........................................ 24

2.4 Metabolic Importance of Amino Acids in Developing Embryos ........................................... 26

2.5 Metabolic Importance of Vitamins in Developing Embryos............................................... 28

2.6 Metabolic Importance of Steroids and Hormones in Developing Embryos .......................... 29

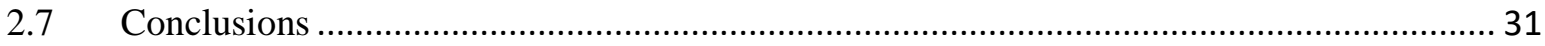

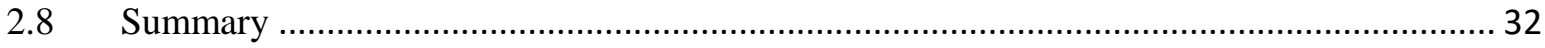

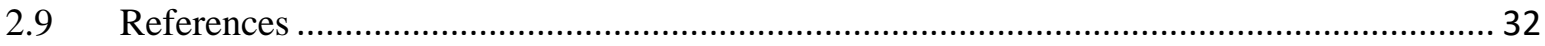

CHAPTER 3 STATISTICAL AND GENETIC ANALYSIS OF HATCHABILITY TRAITS IN

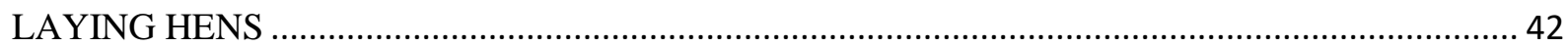

3. Statistical and Genetic Analysis of Hatchability Traits in Laying Hens ................................... 43

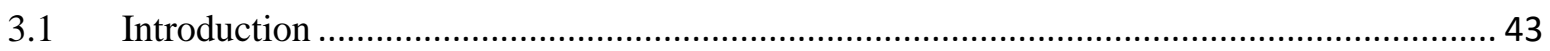

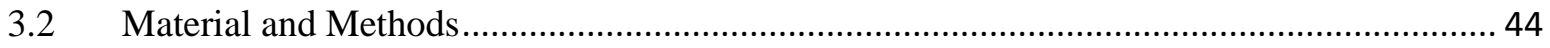

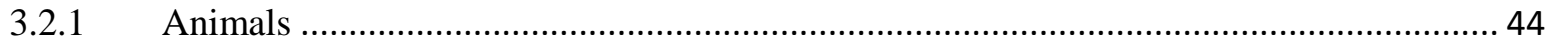

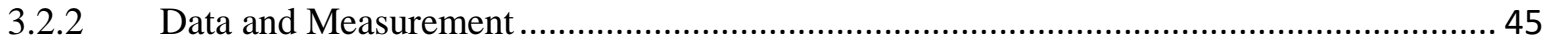

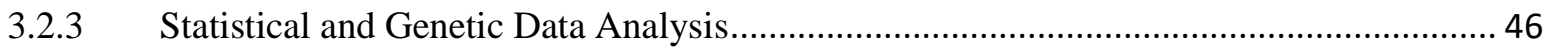

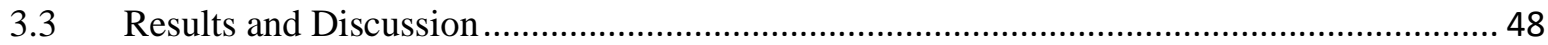

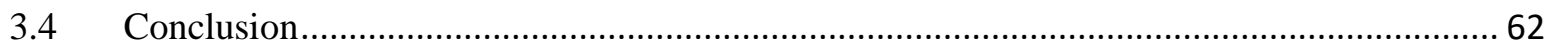

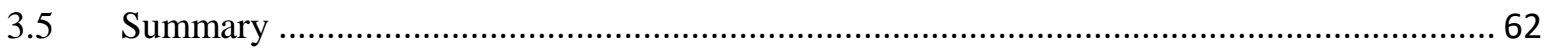


CHAPTER 4 METABOLITE PROFILE OF CHICKEN EGG YOLK AND ITS ASSOCIATION

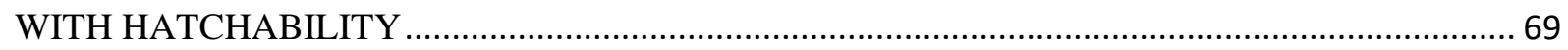

4. Metabolite Profile of Chicken Egg Yolk and Its Association with Hatchability ...................... 70

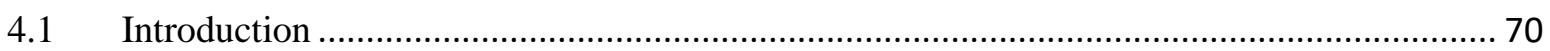

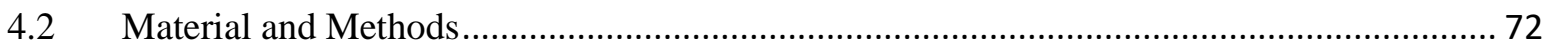

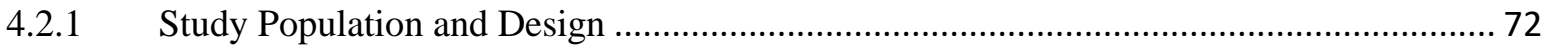

4.2.2 The Determination of Metabolite Profile in Egg Yolk and Data Processing ................... 73

4.2.3 Data Normalization and Association Analysis .............................................................. 74

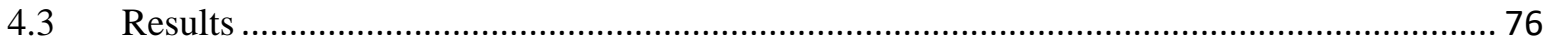

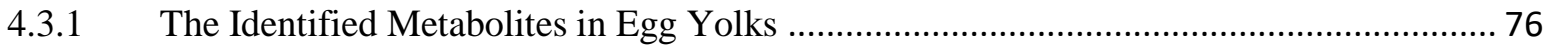

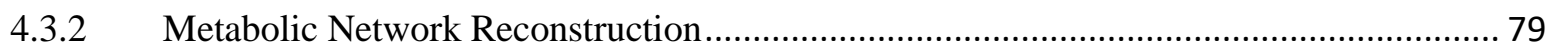

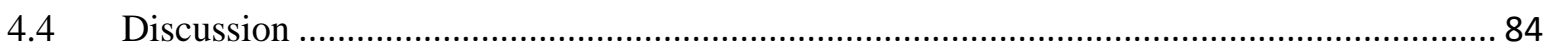

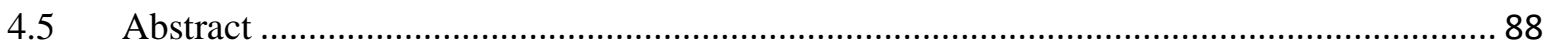

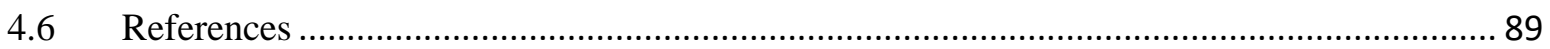

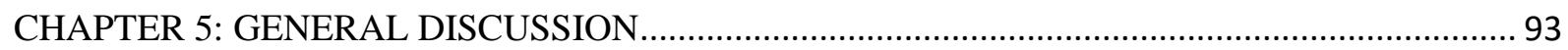

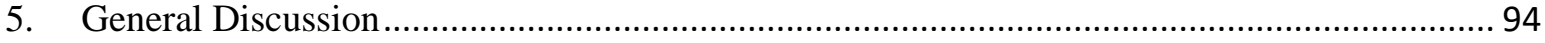

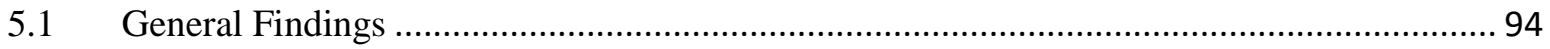

5.1.1 Chicken Embryonic Survival Ability and Impact Factors............................................... 94

5.1.2 Genetic Parameters of Hatching Traits.......................................................................... 96

5.1.3 Impact of Egg Yolk Metabolites on Embryonic Development ........................................ 98

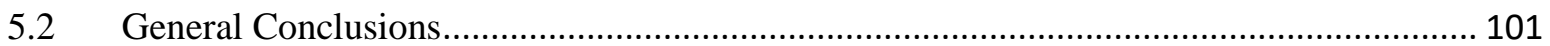

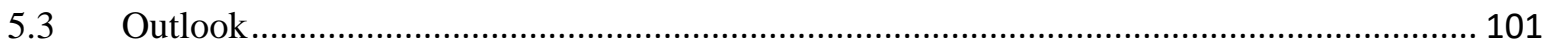

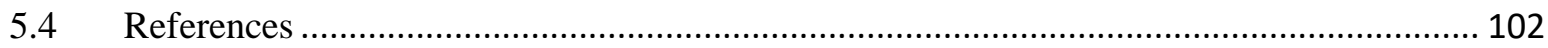

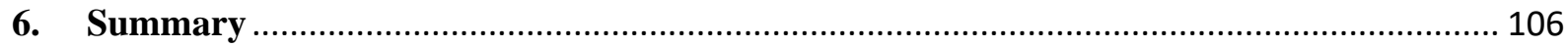




\section{CHAPTER 1 GENERAL INTRODUCTION}




\section{Chapter 1}

\section{General Introduction}

Fertility and hatchability are important traits in breeding programs and have a substantial economic relevance for franchise hatcheries. Improving hatchability by even 1 percent will result in enormous savings of biomass, higher costs of energy and waste disposal, and it also will be a major improvement from the animal welfare point of view[1]. The frequency of embryonic death is not equally distributed over the course of incubation and can be distinguished in three distinct stages: early embryonic mortality occuringduring the first week of incubation, middle embryonic mortality occuringafter the first week of incubation and before transfer of the eggs into hatcher, and late embryonic mortality[2]. These stages are characterized by divergent physiological changes. Besides, different embryonic development stages have distinct causes of embryonic mortality, such as nutritional deficiencies, health of breeder flock, hatching technology, egg quality and genetics [3]. The main goal of this thesis is to estimate genetic parameters of hatching traits and to determine the important metabolites for hatchability. The results found in this research couldbe applied in selecting and breeding of high hatching animals. In this chapter, we give a short overview of the physiology of the chicken embryonic development process followed by the introduction of relevant factors which impact embryonic survival.

\subsection{Chicken Embryonic Development Process}

Hamburger and Hamilton described a series of stages of the normal development of the post oviposition chick embryo (21d)[4]. They defined 3 main phases: early phase (days 1 to 7), middle phase (days 8 to 17), and late phase (days 18 to 21)[4]. Essentially, the germ of a chicken embryo is established during the early phase of incubation, and the formation of organs and systems follows through the middle phase[4, 5, 6]. Preparation for hatching or emergency becomes the objective during the final incubation phase[4, 5, 6]. In Figure 1.1, we present the physiological and metabolic changes occurring at different stages of chicken embryonic development. The early phase is characterised by the formation of egg compartments (amnion, chorion, allantois, and yolk sac) that support embryonic growth[5, 6]. During this time, oxygen supply may be insufficient due to immature blood cells and an underdeveloped chorionic vascular system, so metabolic energy depends on 


\section{Chapter 1}

anaerobic glycolysis of the limited amounts of carbohydrate reserves in the egg. The middle phase of embryonic development is characterised by the establishment of chorioallantoic respiration[5, 6]. A fully developed chorioallantois is seen soon after 7 days of incubation, by which time it is able to assure adequate $\mathrm{O} 2-\mathrm{CO} 2$ exchange and support rapid embryonic growth[5, 6]. Chick embryos are structurally complete at around 14 days of incubation when the major regions of the embryo (such as limbs and visceral arches) are well formed, and then a stage of plateauing oxygen consumption (maximum chorioallantoic respiration capacity) ensues[5, 6]. The final phase is characterised by dramatic physiological and metabolic changes, such as initiation of pulmonary respiration and accumulation of glycogen reserves in muscle and liver tissues[5, 6]. Internal pipping (Day 19) initiates emergence by piercing the chorioallantois and inner shell membrane of the air cell $[5,6,7]$. A concurrent transition to pulmonary respiration occurs in order to assure embryonic oxygen consumption[5, 6, 7]. Pipping movement is driven by the hatching muscle and is facilitated by the beak and body rotation. Hatching muscle fibres most active during pipping exclusively use glycolysis from glucose provided from glycogen reserves[5, 6, 7]. The transient increase of lactic acid occurring at this time disappears once pulmonary functioning provides adequate oxygen for fatty acids catabolism to resume as a source of energy[5, 6, 7]. Besides, plasma thiiodothyronine (T3) and theyroxine (T4) levels peak the day before hatching until the end of incubation[5, 6, 7, 8]. Elevated thyroxine levels are considered crucial for stimulating a variety of developmental and metabolic processes of hatching $[5,6,7,8]$.

Current information on poultry embryo development towards hatching emphasises several pathways that are associated with energy metabolism during this period, including glycolysis, gluconeogenesis, glycogenesis, glycogenolysis and fatty acids $\beta$-oxidation. Most of the challenges which chicken embryos encounter in the first and last phases of incubation are associated with low oxygen availability. This is because, during these phases, the embryos can only utilise glucose and other carbohydrates as their main energy source, and glycolysis and gluconeogenesis pathways are very active[5, 6]. Through glycolysis, glucose can be reduced to 2 pyruvate units plus 6 moles of ATP. When glucose contained within eggs is insufficient, gluconeogenesis occurs and produces glucose 


\section{Chapter 1}

from other carbon-rich components, such as amino acids, glycerol and other carbohydrates $[5,6]$. Furthermore, in the days that precede pipping, glucose derived from amniotic fluid and possibly muscle proteins by gluconeogenesis is utilised to accumulate glycogen reserves through glycogenesis in the liver and muscle[5]. The embryos rely on stored glycogen for muscle activity, heat production, and body maintenance during and after pipping or emergency, until feed is consumed. Once the embryo depletes its glycogen reserves, hatchling vigour and survival are compromised. Homeostatical enzymes of glycogenesis and glycogenolysis are regulated by hormones and other enzymes to ensure a constant supply of glucose to tissues during the final period of incubation[5]. During the middle stage of incubation, as the embryos diminish supply of oxygen limits, energetic metabolism switches from using glucose to yolk lipids. Therefore, fatty acids $\beta$-oxidation pathway is of great importance and most active during this period[5, 6]. Within this metabolic pathway, Acetyl-Coa is produced by fatty acids oxidation in a process similar to the reverse of fatty acids elongation, and subsequently enters the TCA cycle to produce ATP by the enzyme citrate synthase[5, 6].

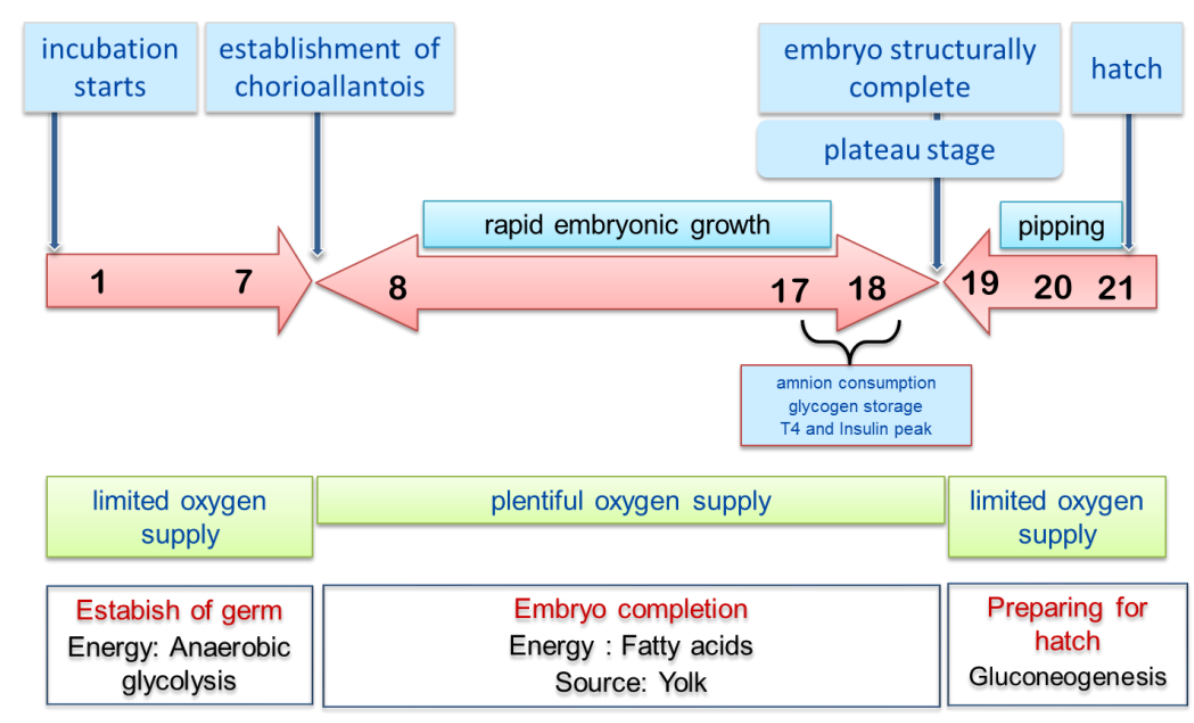

Figure 1.1: Chicken embryonic development periods and their main physiological and metabolic changes (from De Oliveira et al., 2008).

\subsection{Factors Affecting Chicken Embryonic Development}

Knowledge of the normal stages in chicken embryonic development gives a deeper understanding of the developmental process and how embryonic growth and the hatching process can be influenced and 


\section{Chapter 1}

manipulated by both endogenous and exogenous factors. These factors include incubation conditions, genetic abnormality, selection, malposition and insufficient nutrients in the egg that do not meet the needs of the developing embryo. In this section, we will briefly introduce previous researches into these factors' impact on chicken embryo survival. Besides, in chapter 3, we will describe experiments to determinate the effect of genetic abnormality, selection and malposition on hatchability, while in chapter 4 ,we will describe the measurement of the yolk metabolites component and discusses the relationship with embryonic development.

\subsubsection{Incubation Conditions}

In chicken embryos, the heat necessary for development and maintenance of normal metabolic functions is provided by an external source (hen or incubator)[9]. Therefore, temperature influences embryonic growth rate and utilisation of egg yolk and albumen nutrients. However, it may have a divergent effect during the different stages of incubation. Raising temperature initially accelerates metabolic rate of yolk and albumen mobilisation and consumption and, subsequently, embryonic development during incubation, but as incubation progresses, a consistently high temperature slows embryonic growth $[9,10]$. It has been reported that prior to pipping, both chicken and duck embryos show decreased metabolic rates if the internal egg temperature exceeds $40.0^{\circ} \mathrm{C}[9,11]$. Consequently, it is important to maintain a temperature in incubator that optimizes hatchability, currently defined as being between 37 and $38^{\circ} \mathrm{C}[9,12,13,14]$.

As embryonic development progresses, the egg loses water through evaporation concurrently with absorption and metabolism of egg yolk nutrients by the embryo. The rate of incubational water loss influences embryogenesis, and the total water loss has an effect on the creation of air cells sufficient to allow embryonic lung ventilation after internal pipping $[9,13,15]$. Both excessive water loss (higher than $18.5 \%$ ) and insufficient water loss (lower than 9.1\%) can result in a high incidence of embryonic mortality $[9,13]$. The optimum egg water loss is most commonly defined as being $12 \%$ by 18 days of incubation [9]. Dehydration can occur when relative humidity $(\mathrm{RH})$ during incubation or hatching is low, or a long period of time passes between hatching and removal from the hatcher $[9,13,15]$. A 


\section{Chapter 1}

wide range of optimal $\mathrm{RH}$ has been established, between $40 \%$ and $70 \% \mathrm{RH}$, and with maximum hatchability obtained at approximately $50 \% \mathrm{RH}[9,16]$.

Egg storage is a common practice in broiler parent and grandparent hatcheries in order to coordinate hatchery activities and anticipate demand. When in cool storage, eggs are maintained at a temperature well below physiological zero, defined as $21^{\circ} \mathrm{C}$ by Edwards (1902) and $27^{\circ} \mathrm{C}$ by Funk and Biellier (1944)[17, 18, 19]. Storing eggs before incubation can improve embryonic development through reducing weight loss during incubation [20]. Normally, storage of about 10 days is optimal for decreasing embryonic mortality [20]. However, storage of more than 14 days can reduce hatchability significantly [20]. It has been suggested that prolonged egg storage may induce embryonic stress, manifested in increased embryonic necrotic and apoptotic cell death, depressed embryonic metabolism, and developmental delays. As a result of that, embryos may suffer irreparable damage thus causing increased embryonic mortality and decreased chicken performance [21, 22].

Egg turning during incubation is another important incubation factor for chicken embryonic development [23, 24]. This is essential in ensuring the optimum development of embryonic membranes and the formation of correct embryonic position or orientation within the egg during the period immediately prior to hatching[25]. Several authors have reported that turning eggs 24 times daily resulted in better hatchability compared to less frequent turning[25, 26, 27]. In addition, raising turning frequency to 96 times per day from day 3 to day 11 of incubation produces better results of embryonic development and hatching performance than turning frequencies of 24 and 48 times per day during this time[26].

\subsubsection{Genetic Abnormality}

Besides environmental factors, embryonic death, especially occurring in the first 6 days of incubation, often relates to genetic abnormalities, the presence of autosomal or sex-linked recessive lethal genes and chromosome aberrations [28, 29, 30, 31]. Many reports claim that chromosomal abnormalities can cause remarkable (1\% to $12 \%)$ embryonic death during incubation [32, 33]. These disorders are mainly numerical alterations such as triploidy, haploidy or polyploidy, which occur during meiosis or 


\section{Chapter 1}

fertilisation as well as at the beginning of embryonic development [32, 34, 35]. In domestic fowl variation of the ratio of chromosome abnormalities was found among different breeds, families and individuals [36, 37, 38, 39]. Bloom [40] studied 4182 embryos of different breeds. He found chromosome number alteration in proportions of $2.5 \%$, and ranging between $0.4 \%$ and $8.9 \%$ in different breeds. In addition, Lodge et al. [41] found fewer chromosomal alterations (1.4\%) in Leghorn chicken stocks, and that the ratio of embryonic chromosomal abnormalities was higher in heavy weight groups than in lighter groups [42]. Wolowodiuk et al. [37] reported the same phenomenon, where this ratio was $8.9 \%$ in the embryos of heavier birds and $4.1 \%$ in the group with lower weight.

Recessive genes have been proven to cause hatch losses during the early stage of embryonic development. The earliest reported lethal gene is named creeper $(\mathrm{Cr})[43,44]$. This gene is not a completely dominant gene, and the homozygous form is a threat to embryonic viability. Another gene associated with embryonic death was identified by Sheridan [45], named lady-killer (lk), causes the death of female embryos in the first week of incubation. Savage et al. [30] reported an autosomal recessive genetic mutation named blr gene that produces a blood ring and uncoalesced blood islands around the embryo and is associated with the absence of vitelline arteries and a sinus terminalis engorged with erythrocytes. The blr gene is present in Leghorn and New Hampshire populations at frequencies of $0.08 \%$ and $0.14 \%$, respectively[30].

\subsubsection{Selection Effect}

Previous studies carried out on layer populations have shown that chicken fertility and embryonic mortality were affected by intensive selection for layer body weight, egg number, egg weight and egg mass, due to a genetic correlation between laying performance and hatchability $[46,47,48]$. Sewalem et al. [48] reported that, compared to selection for egg number and egg mass, selection for increased egg weight has a more profound effect on fertility decline over generations. Furthermore, selection for low body weight of a layer compared to selection for high weight, results in the production of eggs with superior embryonic development at oviposition and higherhatchability [49, 50]. Body, heart, liver, pipping muscle growth rate as well as glycogenolysis activity of the liver, heart and muscle in externally pipped embryos in a line selected for egg production was lower than in a random control 


\section{Chapter 1}

line $[51,52,53,54]$, because the thyroid hormones play an essential role in the synthesis of glycogen and its accumulation in liver, heart and muscles [55]. The selection for laying performance influencing embryonic metabolic pathways resulting in a retardation of the growth of organs and muscles involved in the hatching process which in turn affects the pipping and embryonic survival.

\subsubsection{Malposition}

Throughout embryonic development towards hatching, chicken embryos progress through a series of positions and finally end in a normal position for hatching. The optimum position is most commonly with the long axis of the body aligned with the long axis of the egg. The head is curled forward and to the right with the beak tucked under the right wing and the tip of the beak pointed toward the air cell in the large end of the egg [56]. The legs are flexed and tucked against the abdominal wall with the abdomen (including the internalized yolk) between the thighs. However, many positions that are associated with difficulty in hatching, also called embryonic malposition, result in increased incidence of embryonic mortality.Landauer [57] classified chicken embryonic malposition into 7 types. Figure 1.2 presents these malpositions and the normal position of chicken embryos. A higher incidence of malposition III (head under left wing) and VI (head over right wing) was observed in a group of embryos in abnormal position, irrespective of the investigated breeding lines. Malposition III (headunder-left-wing) has been described as having a highly deleterious effect on hatchability [57, 58, 59]. Landauer [57] and Purohit [58] showed that position III was more lethal in most cases. However, the high incidence of malposition VI may simply reflect a normal variant position as suggested by Wilson [60]. To reveal the cause of malposition, Wilson [60] conducted experiments into a lack of egg turning. He confirmed that insufficient egg turning can induce adhesion of the embryo to the inner shell membrane and result in malposition. He also states that nutritional deficiencies, especially in vitamin E or selenium, increase the probability of malposition associated with a swollen head and back of neck (exudative diathesis-increased capillary permeability). 


\subsubsection{Chicken Egg Yolk Metabolites and Embryonic Nutrition}

The egg yolk can be considered as a primary capsule of life, in which all ingredients are incubated in the precise amounts necessary to sustain development of an embryo and give rise to the birth of a chick [61]. The supply of nutrients in egg yolk originates in the maternal diet and metabolism [62]. Maternal nutrition deficiencies may affect the embryos of hens and may result in predominantly early and late incubation deaths or other late occurring effects [62]. Besides, it is likely that several other genetic factors also influence embryonic nutrient supplies or deposition from the hen [63].

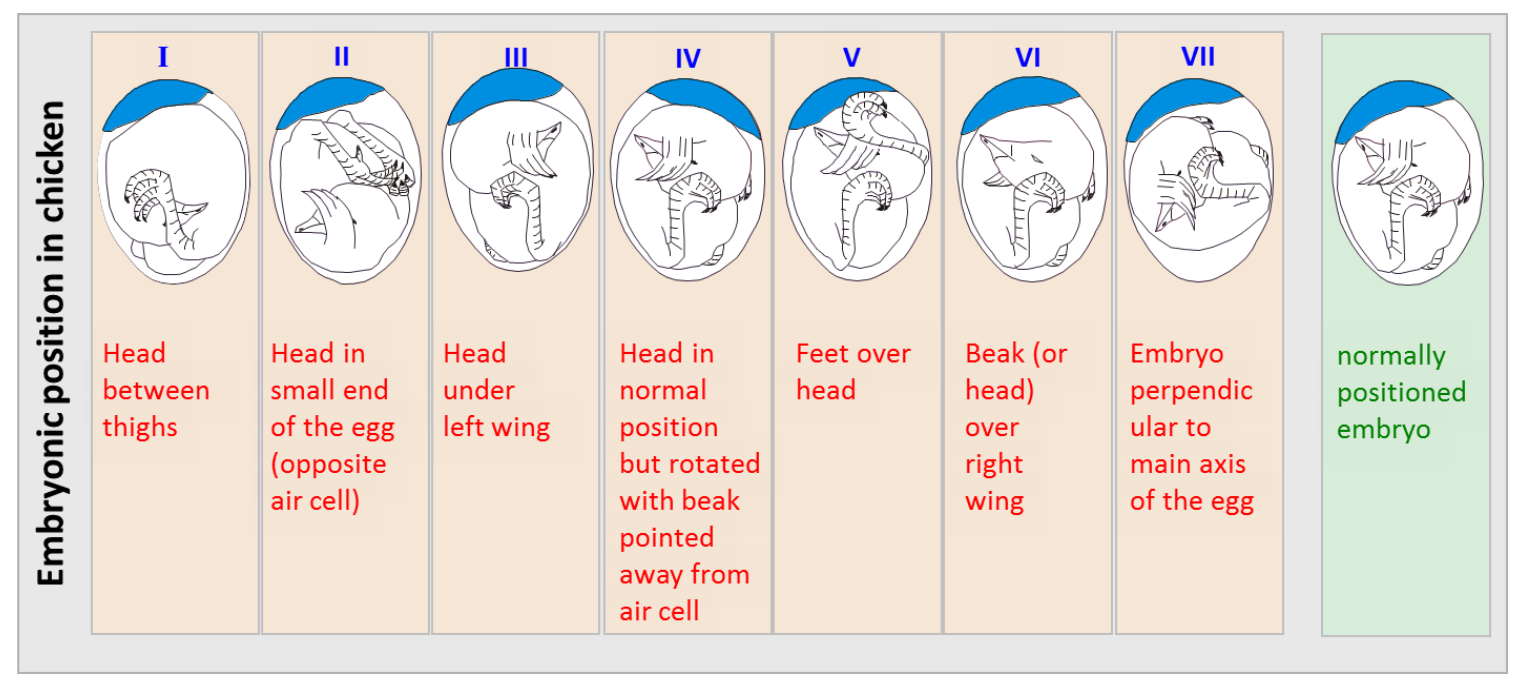

Figure 1.2: Chicken embryonic positions in the late stage of incubation. 7 types of malposition and normal embryonic position (from Landauer, 1967).

Poultry egg yolk makes up approximately $36 \%$ of the total fresh chicken egg mass and contains the majority of the nutrients. Lipids are the main component of the yolk (approximately 65\% of the dry matter), with protein only accounting for approximately 33\% [64]. The fatty acids composition of the lipids consists of approximately 30\% - 35\% saturated fatty acids (SFAs), 40\% - 45\% monounsaturated fatty acids (MUFAs), and 20\% - 25\% of polyunsaturated fatty acids (PUFAs) [64].Synthesised yolk fats in maternal liver are transported and deposited to egg yolk through serum via triacylglycerol-rich, very low density lipoprotein (VLDL) (Figure 1.3). VLDL targeted at yolk is about half the size of normal VLDL, and is a specialised form of VLDL that is specific to laying hens called VLDLy. 


\section{Chapter 1}

VLDLy is composed of ApoB100 and apovitellenin-1 (apoVLDL-II) and is not be acted upon by lipoprotein lipase (LPL)[65]. This characteristic allows triglycerides to be deposited to the oocyte intact. In order to control the fatty acids composition of the yolk, no exogenous lipids are transported from the liver to the yolk, only de novo triglycerides are packaged into VLDL for transport[65].

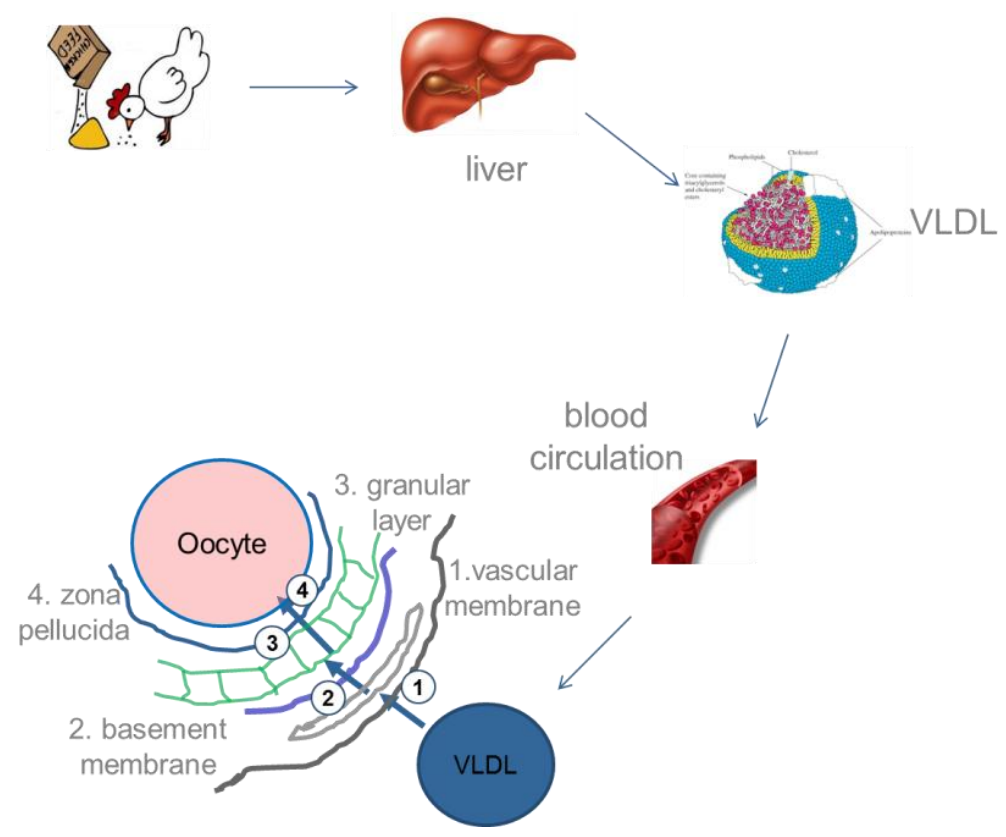

Figure 1.3: Chicken egg yolk lipids deposition through VLDL transportation system (from LópezFandiño et al., 2007).

Dramatic alteration in nutrient composition of egg yolk may induce a series of embryonic metabolic disorders manifested in decreased nutrient uptake rate, depressed energy metabolism, and retarded embryonic development in specific organs such as muscles and the liver. The loss of egg survival ability is highly associated with a 2 to 3 -fold increase in desaturated ratios (cis-9 C16:0/C16:1 and cis$9 \mathrm{C} 18: 0 / \mathrm{C} 18: 1)$ in the total egg yolk lipids[66]. Dietary conjugated linoleic acid (CLA) has been reported to increase the ratio of $\mathrm{C} 16: 0 / \mathrm{C} 16: 1$ and $\mathrm{C} 18: 0 / \mathrm{C} 18: 1$ by inhibiting stearoyl-CoA desaturase enzyme activity which catalyses the insertion of a double bond between the C-9 and C-10 atoms of C16:0 or C18:0 in the formation of C16:1(n-7) and C18:1(n-9), respectively[66]. Average ratios of C16:0/C16:1 in yolk from groups fed 0, 0.5, 1\% CLA were 8.2\%, 20.4\% and 24.6\%, respectively[66]. Concurrently, average hatchability of eggs from groups fed $0,0.5,1 \%$ CLA were $84 \%, 64 \%$ and $0 \%$, respectively[66]. Since C16:1, C16:0, C18:1 and C18:0 are the most prevalent fatty acids within egg 


\section{Chapter 1}

yolk and constitute more than $80 \%$ of total fatty acids in the egg, changes in the ratios of C16:0/C16:1 and $\mathrm{C} 18: 0 / \mathrm{C} 18: 1$ result in alteration of the ratio of saturated fatty acids (SFAs) to monounsaturated fatty acids (MUFAs), and subsequently interfere with the absorption of yolk fatty acids through the yolk sac membrane in developing embryos, causing embryonic mortality to ensue[66, 67].

Other than that, it is essential to establish a stable and sufficient cell membrane polyunsaturated fatty acids (PUFAs) status during incubation in order to maintain general metabolism and healthy immunity of chicken embryos. Cell membrane phospholipids are rich in long chain PUFA. Among these PUFAs, Arachidonic acid (ARA) and Eicosapentaenoic acid (EPA) are potentially biologically active precursors and can be mobilised by cyclooxygenase or lipoxygenase to generate eicosanoids, such as prostaglandins (PG), thromboxanes (TX) and leukotrienes (LT), which in turn serve as important lipid mediators in the process of inflammation[65]. It has been reported that maternal nutritional deprivation or early dietary deficiency of these PUFAs may impair eicosanoid production in immune cells and vital organs in current commercial hatching eggs[65]. Besides, Eicosanoids concentration in the tissues of hatched chickens increases when feeding hens sunflower oil (n-6 PUFAs enriched diet) rather than fish oil (n-3 PUFAs enriched diet)[65, 68, 69]. Concurrent with that, the ratios of EPA to ARA and the ratio of leukotrienes B5 (LTB5) to leukotrienes B4 (LTB4) in tissues are higher in chickens hatched from n-3 PUFA enriched eggs[65, 68, 69]. Since Eicosanoids derived from arachidonic acid such as LTB4, PG2, and TX2 are more pro-inflammatory than those derived from EPA (e.g., LTB5, PG3,TX3), it seems to indicate that chickens prefer not to use EPA as precursors for eicosanoids generation, but to utilise ARA to produce them[65. 70].

\subsection{Genetic Parameter Estimation Studies}

Preciously, many researchers have conducted genetic parameter estimation for hatching traits. [71, 72, $73,74,75,76]$. Beaumont et al. [73] pointed to the various factors that influence different incubation stages. There was no doubt that the differing mortality rates in the 3 stages were due to different causes as previously mentioned [73]. Therefore, the estimated genetic parameters reflected the genetic background of different physiological pathways acting at different stages of embryonic development. Beaumont et al. [73] estimated the genetic components from a sire-dam model for different stages of 


\section{Chapter 1}

embryonic development. The estimated heritabilities based on the sire component for fertility (FER), early embryonic survival ability (EES), middle embryonic survival ability (MES), late embryonic survival ability (LES), hatchability of fertilised eggs (HFE) were 0.09, 0.09, 0.07, 0.05 and 0.05, respectively. However, heritabilities of FER, EES, MES, LES, and HFE estimated from the dam model were $0.31,0.25,0.20,0.18$ and 0.15 , respectively [73]. Bennewitz et al. [75] applied the Bayesian threshold model for a genetic evaluation of reproductive traits in White Leghorn layers and found that the estimated heritability ranging from 0.06 to 0.14 . They showed that the obtained heritability estimated was higher compared with their expected values obtained from linear models. Former researches on genetic correlation showed that fertility was not correlated to HFE, whereas fertility was positively correlated to HTE $[71,73,74,77]$. Both Sewalem et al [74] and Beaumont et al. [73] indicated selection for increased fertility or hatchability should result in reduced embryonic death. Furthermore, Beaumont et al. [73] illustrated that estimates of genetic correlations between fertility rate and susceptibility to the different stages of embryonic death were generally favourable. Chapter $\mathbf{3}$ describes a generalised linear mixed model which was applied to estimate genetic parameters in 4 lines, and a comparison with the results of other researchers was conducted.

\subsection{References}

1. Sharifi AR, Zhang Y, Weigend S, Weigend A, Preisinger R, Schmutz M, Römisch-Margl L, Jonczyk R, Gülersönmez MC, Piepho HP, Malik WA, Sitzenstock F, Simianer H. Association between Metabolites in Egg Yolk and Hatchability Traits in Laying Hens. Proc 10th World Congr Genet Appk Livest Prod, Vancouver, Canada; 2014.

2. Beaumont C, Millet N, Le Bihan-Duval E, Kipi A, Dupuy V. Genetic parameters of survival to the different stages of embryonic death in laying hens. Poultry Sci 1997;76:1193-1196.

3. Liptoi K, Hidas A. Investigations of possible genetic background of early embryonic mortality in poultry. World Poultry Sci J 2006;62:326-337.

4. Hamburger V, Hamilton HL. A series of normal stages in the development of the chick embryo. Dev Dynam 1992;195:231-272. 


\section{Chapter 1}

5. De Oliveira JE, Uni Z, Ferket PR. Important metabolic pathways in poultry embryos prior to hatch. World Poultry Sci J 2008;64:488-99.

6. Moran ET. Nutrition of the developing embryo and hatchling. Poultry Sci 2007;86:1043-9.

7. Lu JW, Mcmurtry JP, Coon CN. Developmental changes of plasma insulin, glucagon, insulin-like growth factors, thyroid hormones, and glucose concentrations in chick embryos and hatched chicks. Poultry Sci 2007;86:673-683.

8. McNabbFM. The hypothalamic-pituitary-thyroid (HPT) axis in birds and its role in bird development and reproduction. Crit Rev Toxicol 2007;37:163-193.

9. Noiva RM, Menezes AC, Peleteiro MC. Influence of temperature and humidity manipulation on chicken embryonic development. BMC veterinary research 2014;10: 234.

10. Romanoff AL. Pathogenesis of the Avian Egg. Wiley-Inter-science, New York, NY 1972.

11. Janke O, Tzschentke B, Hochel J, Nichelmann M. Metabolic responses of chicken and muscovy duck embryos to high incubation temperatures. Comp Biochem Physiol A Mol Integr Physiol $2002 ; 131: 741-750$.

12. Fasenko GM, Wilson JL, Robinson FE, Hardin RT. Effects of Length of Egg Nest Holding Time and High Environmental Temperatures on Prestorage Embryonic Development, Survival, and Hatchability of Broiler Breeders. J Appl Poultry Res 1999;8:488-492.

13. van der Pol CW, Van Roovert-Reijrink IAM, Maatjens CM, van den Brand H, Molenaar R: Effect of relative humidity during incubation at a set eggshell temperature and brooding temperature posthatch on embryonic mortality and chick quality. Poultry Sci 2013;92:2145-2155.

14. Yalcin S, Siegel PB. Exposure to cold or heat during incubation on developmental stability of broiler embryos. Poultry Sci 2003;82:1388-1392.

15. Burnham MR, Peebles ED, Gardner CW, Brake J, Bruzual JJ, Gerard PD. Effects of incubator humidity and hen age on yolk composition in broiler hatching eggs from young breeders. Poultry Sci 2001;80:1444-1450.

16. Bruzual JJ, Peak SD, Brake J, Peebles ED. Effects of relative humidity during incubation on hatchability and body weight of broiler chicks from young breeder flocks. Poultry Sci 2000;79:827-830. 


\section{Chapter 1}

17. Edwards, C. L. The physiological zero and the index of development for the egg of the domestic fowl (Gallus domesticus). Am J Physiol 1902;6:351-397.

18. Funk EM, Biellier HV. The minimum temperature for embryonic development in the domestic fowl (Gallus domesticus). Poultry Sci 1944;23:538-540.

19. Dymond J, Vinyard B, Nicholson AD, French NA, Bakst MR. Short periods of incubation during egg storage increase hatchability and chick quality in long-stored broiler eggs. Poultry Sci 2013;92: 2977-2987.

20. Romao JM, Moraes TGV, Teixeira RSC, Cardoso WM, Buxade CC. Effect of egg storage length on hatchability and weight loss in incubation of egg and meat type japanese quails. Braz J Poultry Sci 2008;10:143-147.

21. Fasenko GM. Egg storage and the embryo. Poultry Sci 2007;86:1020-1024.

22. Hamidu JA, Rieger AM, Fasenko GM, Barreda DR. Dissociation of chicken blastoderm for examination of apoptosis and necrosis by flow cytometry. Poultry Sci 2010;89:901-909.

23. Wilson HR. Physiological requirements of the developing embryo: Temperature and turning in avian incubation. Tullet Butterworth-Heinemann London UK 1991:145-156.

24. Eycleshymer AC. Some observations and experiments on the natural and artificial incubation of the egg of common fowl. Bio Bull 1906;12:360-374.

25. Elibol O, Brake J. Effect of egg position during three and fourteen days of storage and turning frequency during subsequent incubation on hatchability of broiler hatching eggs. Poultry Sci 2008;87: 1237-1241.

26. Robertson, IS. The influence of turning on the hatchability of hens' eggs. I. The effect of rate of turning on hatchability. J Agric Sci Camb 1961;57:49-56.

27. Deeming, DC. Characteristics of unturned eggs: Critical period, retarded embryonic growth and poor albumen utilization. Br Poult Sci 1989;30:239-249.

28. Zartman DL, Smith AL. The effect of five different chromosome mutations on embryonic survival studied in chicken. Genetics 1975;80:87-88.

29. Somes RG, Smyth JR. Prenatal, a sex-linked lethal mutation of the fowl. J Hered 1967;58:25-29. 


\section{Chapter 1}

30. Savage TF, Defrank MP, Brean SE. Blood ring: An early embryonic lethal condition in chicken. J Hered 1988;79:124-128.

31. Savage TF, Mirosh LW, Jones JL, Schneiderman ET. Blastoderm degeneration. an early embryonic failure in dwarf single comb white leghorn chickens. J Hered 1992;83:249-254.

32. Fechheimer NS. Origins of heteroploidy in chicken embryos. Poultry Sci 1981;60:1365-1371.

33. Szalay I, Hidas A, Vas E. Study on the frequency of chromosomally abnormal embryos in broiler chicken grandparent stocks. (in Hungarian) Baromfitenyésztés és feldolgozás 1988;35:149-155.

34. Mong SJ, Snyder MD, Fechheimer NS, Jaap RG. The origin of triploidy in chick (Gallus domesticus) embryos. Can J Genet Cytol 1974;16:317-322.

35. Fechheimer NS, Jaap RG. Origins of euploid chimerism in embryos of Gallus domesticus. Genetica 1980;52/53:69-72.

36. Reddy PRK, Siegel PB. Chromosomal abnormalities in chickens selected for high and low body weight. J Hered 1977;68:253-256.

37. Wolowodiuk VD, Fechheimer NS, Nestor KE, Bacon WL, Chromosome abnormalities in embryos from lines of Japanese quail divergently selected for body weight. Genet Sel Evol 1985;17:183190.

38. Thorne MH, Collins RK, Sheldon BL. Live haploid-diploid and other unusual mosaic chickens (Gallus domesticus). Cytogenet Cell Genet 1987;45:21-25.

39. Thorne MH, Sheldon BL. Cytological evidence of maternal meiotic errors in a line of chickens with a high incidence of triploidy. Cytogenet Cell Genet 1991;57:206-210.

40. Bloom SE. Chromosome abnormalities in the chicken (Gallus domesticus) embryos: Types, frequencies and phenotypic effects. Chromosoma 1972;37:309-326.

41. Lodge JR, Fechheimer NS, Miller RC. Deletion, monosomy and multiple monosomy-trisomy in chicken embryos. Poultry Sci 1973;52: 397-399.

42. Jaszczak K, Sadowska G, Pawluczuk B. Chromosomal abnormalities in quails selected for a high and low body weight. Genet Pol 1985;25:417-425.

43. Landauer W, Dunn NC. Studies on the creeper fowl. J Genet 1930;23:37-53. 


\section{Chapter 1}

44. Landauer W. Studies on the creeper fowl. III. The early development and lethal expression of homozygous creeper embryos. J Genet 1932;25:367-394.

45. Sheridan AK. Further studies with a sex-linked lethal gene in the fowl. Brit Poultry Sci 1979;20:571-573.

46. Rozempolska-Rucińska I, Zięba G, Twardowska M, Łukaszewicz M, Witkowski A. Relationships between hatchability and routine selection criterion in laying hens. Proc 19th Int Poultry Sci PB WPSA Olsztyn 2007.

47. Rozempolska-Ruciska I, Zieba G, Łukaszewicz M. Hatchability traits as selection criteria in breeding of laying hens. Arch Geflugelkd 2008;73:263-267.

48. Sewalem A, Johansson K, Carlgren AB, Wilhelmson M, Lillpers K. Are reproductive traits impaired by selection for egg production in hens?. J Anim Breed Genet 1998;115:281-297.

49. Christensen VL, Wineland MJ, Fasenko GM, Donaldson WE. Egg storage effects on plasma glucose and supply and demand tissue glycogen concentrations of broiler embryos. Poultry Sci 2001;80:1729-1735.

50. Coleman JW, Siegel PB. Selection for body weight at eight weeks of age: Embryonic state at oviposition and its relationship to hatchability. Poultry Sci 1966;45:1008-1011.

51. Christensen VL, Donaldson W E, Nestor KE. Effect of supplemental oxygen on blood plasma organic acids within embryos from selected lines of turkeys. Poultry Sci 1999;78:1601-1605.

52. Christensen VL, Donaldson WE, Nestor KE, McMurtry JP. Effect of genetics and maternal dietary iodide supplementation on turkey embryonic growth. Poultry Sci 1999;78:883-889.

53. Christensen VL, Donaldson WE, Nestor KE. Embryonic viability and metabolism in turkey lines selected for egg production or growth. Poultry Sci 1993;72:829-838.

54. Christensen VL, Nestor KE. Changes in functional qualities of turkey eggshells in strains selected for increased egg production or growth. Poultry Sci 1994;73:1458-1464.

55. Christensen VL, Donaldson WE, McMurtry JP. Physiological differences in late embryos from turkey breeders at different ages. Poultry Sci 1996;75:172-178.

56. Ernst RA, Bradley FA, Delany ME, Abbott UK, Craig RM. Egg candling and breakout analysis. ANR Publication 8134, Univ of California 2004. 


\section{Chapter 1}

57. Landauer W. The Hatchability of Chicken Eggs as Influenced by Environment and Heredity. Agricultural Experiment, Storrs Station Monograph 1967;1:68-137.

58. Purohit VD, Basrur PK, Reinhart BS. Malpositions in chicken-pheasant hybrid embryos. Brit Poultry Sci 1974;15:145-151.

59. Byerly TC, Olsen MW. Causes of the embryonic malposition head-under-left-wing. Poultry Sci $1934 ; 13: 278-282$.

60. Wilson HR. Hatchability Problem Analysis. Univ of Florida IFAS Extension 2004.

61. Mine Y. Egg Bioscience and Biotechnology. John Wiley and Sons. 2007.

62. Wilson HR. Effects of maternal nutrition on hatchability. Poultry Sci 1997;76:134-143.

63. Maw AJG. Inherited riboflavin deficiency in chicken eggs. Poultry Sci 1954;33:216-217.

64. López-Fandiño R, Anton M, Schade R. Bioactive Egg Compounds. Berlin: Springer Verlag 2007:33-66.

65. Cherian, G. Nutrition and metabolism in poultry: role of lipids in early diet. J Anim Sci Biotechnol $2015 ; 6(1), 28$.

66. Aydin R, Cook ME. The effect of dietary conjugated linoleic acid on egg yolk fatty acids and hatchability in Japanese quail. Poultry Sci 2004;83: 2016-2022.

67. Noble RC, Cocchi M. 1990. Lipid metabolism and the neonatal chicken. Proc Lipid Res 1990; 29:107-140.

68. Cherian G. Essential fatty acids and early life programming in meat-type birds. World Poultry Sci. 2011;67:599-614.

69. Cherian G, Bautista-Ortega J, Goeger DE. Maternal dietary n-3 fatty acids alter cardiac ventricle fatty acid composition, prostaglandin and thromboxane production in growing chicks. Prostaglandins, Leukot. Essent. Fatty Acids. 2009;80:297-303.

70. Hall JA, Jha S, Skinner MM, Cherian G. Maternal dietary (n-3) fatty acids alter immune cell fatty acid composition and leukotriene production in growing chicks. Prostaglandins Leukot Essent Fatty Acids. 2007;76:19-28.

71. Beaumont C. Comparison of Henderson's method I and restricted maximum likelihood estimation of genetic parameters of reproductive traits. Poultry Sci 1991;70:1462-1468. 


\section{Chapter 1}

72. Beaumont C. Genetic parameters of the duration of fertility in hens. Can J Anim Sci 1992;72:193201.

73. Beaumont C, Millet N, Le Bihan-Duval E, Kipi A, Dupuy V. Genetic parameters of survival to the different stages of embryonic death in laying hens. Poultry Sci 1997;76:1193-1196.

74. Sewalem A, Wilhelmson M. Genetic study of embryonic mortality in white leghorn lines selected for egg production traits. Brit Poultry Sci 1999;40:467-471.

75. Bennewitz J, Morgades O, Preisinger R, Thaller G, Kalm E. Variance component and breeding value estimation for reproductive traits in laying hens using a Bayesian threshold model. Poult. Sci $2007 ; 86: 823-828$

76. Szwaczkowski T, Wezyk S, Piotrowsk Pi, Cywa-Benko K. Direct and maternal genetic and environmental effects for fertility and hatchability in laying hens. Arch Geflugelkd 2000;64:115120.

77. Larivière JM, Michaux C, Leroy $\mathrm{P}$. Genetic parameters of embryonic viability traits in a traditional chicken breed. Int J Poultry Sci 2009;8:1183-1188. 


\section{CHAPTER 2 METABOLITES IN EGG YOLK}

\section{IMPORTANT FOR POULTRY DEVELOPMENT DURING THE INCUBATION PERIOD}


Chapter 2

\section{Metabolites in Egg Yolk Important for Poultry Development during the Incubation Period}

\subsection{Embryonic Development Periods}

Moran [3] summarized the process of embryonic development, dividing it into three major stages. The first stage includes the first week of incubation, in which the egg compartments, including the amnion, chorion, allantois and yolk sac, are formed [3, 17, 18]. However, the oxygen supply during this stage is insufficient due to the lack of mature blood cells and the underdeveloped chorioallantoic vascular system. Thus, the energy for embryonic development comes from the anaerobic glycolysis [3, 19]. In the second stage, lasting from the 8th day to approximately the 17th day, the chorioallantois is fully developed, allowing for adequate $\mathrm{O}_{2}-\mathrm{CO}_{2}$ exchange, and the embryonic growth rate is extremely high. Besides, embryos can utilize aerobic respiration to fully oxidize carbohydrates and the $\beta$-oxidation of fatty acids to supply energy $[1,3]$. The last phase is called emergence and is when the embryos undergo a dramatic physiological change and prepare for pipping out. The oral consumption of the amnion, the accumulation of glycogen reserves in tissues and the initiation of pulmonary respiration are the main characteristics of this stage $[3,20,21]$. Because great metabolic changes are occurring, any disturbance during this phase will lead to embryonic death or influence post-hatching performance $[3,22]$.

\subsection{Metabolic Importance of Fatty Acids in Developing Embryos}

The poultry egg yolk makes up approximately $36 \%$ of the total mass of a fresh chicken egg and contains the majority of the nutrients $[13,23]$. Lipids are the main component of the yolk (approximately $65 \%$ of the dry matter), with proteins only accounting for approximately $33 \%$ of the dry matter $[13,23,24]$. The fatty acid composition of the lipids is approximately $30-35 \%$ saturated fatty acids (SFAs), $40-45 \%$ monounsaturated fatty acids (MUFAs), and $20-25 \%$ polyunsaturated fatty acids (PUFAs) [13, 23], all of which strongly affect embryonic survival ability [1, 2, 5, 13]. Docosahexaenoic acid (DHA, 22:6, n-3), eicosatetraenoic acid (AA, 20:4, n-6) and eicosapentaenoic 


\section{Chapter 2}

acid (EPA, 20:5, n-3), as representative PUFAs, are well known factors involved in the protection of neural system development. Compared with many other avian species, chickens are precocial at hatching. This advanced development at hatch time allows chickens to rapidly become active and mobile, have plumage, open their eyes and have thermolegulatory ability [25]. Then, they are able to leave the nest to find food soon after hatching. This developmental advantage is closely related to prehatch brain maturation in chickens [25]. Large number of double bonds in the long carbon chains of PUFAs such as DHA increases the degree of membrane flexibility, compressibility, elasticity and permeability. These special membrane properties are crucial for neural tissues of brain to accommodate stimulating interactions and interaction-induced conformational changes of membrane protein $[25,26,27]$.

DHA and EPA in the body of chickens mainly come from their diet. The body DHA and EPA synthesis rates are quite small and not sufficient for maintenance of life [25]. The molecular structures of DHA and EPA are similar, especially with respect to the position of their w-3 double bond, and these molecules can transform into each other under certain conditions.Noble and Cocchi [28] found determinations of thechanges in brain lipid composition over the incubation period have shown a specificaccumulation of omeg-3 fatty acid, especially DHA, containing phospholipids to occur particularlyat around the twelfth day. Apart from brain, the retina has a high concentration of omega-3, particularly DHA, which optimizes fluidity of photoreceptor membranes, retinal integrity, and visual function [29].

In birds, insufficient dietary intake of omega-3 fatty acids at crucial stages of development will impede the accretion of DHA-phospholipids. Feeding hens negligible amounts of omega-3 fatty acids leads to marked DHA deficiencies in their eggs and the subsequent reduced DHA in brain appearance of disorders of the brain and the impaired development of other neural tissues in these malnourished chickens $[25,30]$. Reduction of DHA in neuronal phospholipid pools is partially compensated by another unsaturated fatty acid, docosapentaenoic acid (DPA), an omega-6 fatty acid. DPA has a carbon chain of the same length as DHA but does not contain a w-3 double bond. In many cases, an almost complete reciprocal replacement between DHA and DPA can occur. However, this replacement does 


\section{Chapter 2}

not prevent the incidence of visual, cognitive and behavioural impairments caused by a lack of DHAphospholipids $[25,30]$. DPA cannot replace DHA in terms of function of specific neural tissues, such as rhodopsin, as distinguish in sensitivity between DPA-phospholipids and DHA-phospholipids [31]. When rhodopsin is reconstituted in liposomes composed of DPA-phospholipids, phosphodiesterase activity after light activation is significantly less than that in membranes composed of DHAphospholipids [31].The double bond at the w-3 position of DHA likely imparts a special function to the molecule and may be the fundamental factor in maintaining the precise molecular structure of membrane phospholipid fatty acids for the normal functioning of the brain and eyes [32].

In addition, AA and EPA (20:5, n-3) are the precursors of eicosanoids such as prostaglandins (PG), thromboxanes (TX) and leukotrienes (LT). However, eicosanoids derived from AA, such as PGE2, TXB2, and LTB4, are normally proinflammatory and more potent than eicosanoids derived from EPA, such as PGE3, TXB3, and LTB5 [33, 34]. Eicosanoids stimulate biological activities related to platelets, vascular smooth muscle, bronchopulmonary function, gastrointestinal integrity and reproduction [35]. One of the reasons PUFAs are required in the embryonic tissues of developing poultry is to maintain adequate precursors for eicosanoid biosynthesis [24]. Inhibitors of prostaglandin synthetase decrease the number of fibers in chicken embryo skeletal muscles [36], raising the possibility that muscle formation may be capable of being stimulated by PGs [36]. Among PGs, PGE1 may control cell fusion in chick embryo myoblasts, which is dependent upon the synthesis, release and binding of PGs to a receptor [37]. In addition to the responses observed in myoblasts, PGE1 and PGE2 have stimulatory effects on chondrogenesis in vitro. Nagai [38] and Watkins [39] demonstrated that PGE1 stimulates DNA synthesis in chick embryonic chondrocytes in vitro, and PGE2 affects proliferation and collagen production in fibroblasts in a dosage-dependent manner

MUFAs, another large class of unsaturated fatty acids, greatly impact hatchability. Two of these compounds, 9-octadecenoic acid (oleic acid, 18:1, n-9) and 9-hexadecenoic acid (palmitoleic acid, $16: 1, n-7)$, are the most important and make up $40 \%$ of the total fatty acids in egg yolk $[13,23]$. The ratios of $18: 0$ to $18: 1$ and of $16: 0$ to $16: 1$ fatty acids are crucial for embryonic survival $[5,40,41]$. Donaldson and Fites [40] determined that increased stearic acid (18:0) and decreased oleic acid levels 


\section{Chapter 2}

in the diet induce embryonic mortality. Typically, the ratio of 18:0 to $18: 1$ should not exceed 0.25 ; otherwise, hatchability will be adversely affected [41]. Several authors [5, 28, 42] have shown that 18:1 is an important factor for lipid transportation into chicken embryosin the yolk sac membrane. If the embryo cannot achieve a setamount of fatty acid from yolk via yolk sac repackagingand transport, then embryonic growth will be affected. Feeding chickens conjugated linolenic acid (CLA) dramatically changes the $16: 0 / 16: 1$ and 18:0/18:1 ratios in egg yolk, altering the ratio of SFAs to MUFAs, then decreases embryonic survival ability[5, 42]. Aydin et al. [5] and Leone et al. [42] also noted that a high ratio of SFAs to MUFAs may obstruct fatty acid absorption by developing embryos, causing an embryonic energy deficiency. Moreover, oleic acid and other long-chain MUFAs are associated with reduction of blood cholesterol level. Former research found high chicken egg yolk cholesterol content would increase blood cholesterol level in embryos, then decrease hatchability of fertile eggs, and hatchability of total eggs [43]. Rumsey et al. [44] illustrated that these fatty acids play a role in decreasing plasma cholesterol levels by stimulating acyl-CoA:cholesterol acyltransferase (ACAT) activity, which controls cholesterol esterification reaction, and providing feedback to stimulate LDLs receptor activity, then activating cholesterol transportation into cell. However, researchers have found that the intake of excess oleic acid and other MUFAs in humans promotes insulin resistance and increases the risk of many diseases induced by interleukin 1 (IL-1)[45, 46, 47]. IL-1 family is a group of 11 cytokines, which induces a complex network of proinflammatory cytokines and via expression of integrins on leukocytes and endothelial cells, regulates and initiates inflammatory response $[48,49]$. Increased levels of TNF and IL-1 in the brain would induce neural diseases, such as neuroinflammation and cause the breakdown of the blood-brain barrier [48, 49]. But it is still not clear whether in chicken embryos, MUFAs high-fat diets enhance -IL-1-mediated adipose inflammation and insulin resistance.

Branched-chain fatty acids (BCFAs) are a very small component of egg yolk fatty acids [50]. Most of these compounds in egg yolk originate from the maternal diet, which contains animal or plant oils. Many studies have recently observed that BCFAs inhibit tumour cell growth in humans. Yang et al. [51] extracted 13-methyltetradecanoic acid (13-MTD), an iso-C15 SFA, from a soybean product, 
added the compound into a tumour cell culture system, and demonstrated that it could suppress tumour cell proliferation and induce cell apoptosis [51]. Normally, tumour cell growth requires sufficient fatty acids to provide energy and renewable raw materials for cell growth. Thus, the fatty acid biosynthetic pathway is highly active in tumour cells. 13-MTD is one of the inhibitors of this pathway that can effectively block fatty acid generation in tumour cells but has less of an effect on normal cells [52]. Contrary to structural membrane glycerolipids containing straight chain fatty acids, 13-MTD is a fatty acid with branched carbon chain, and can be classified as unusual fatty acid. Incorporation of phospholipids containing 13-MTD into membrane structure influenced the membrane environment and stimulated caspase-independent pathway is the case of tumour cell death stimulus [51, 52, 53]. The terminally branched methyl group may give 13-MTD advantages over straight carbon chain fatty acids to perform the function of apoptotic induction [51, 52, 53]. In chicken embryos, certain types of tumours, such as those associated with lymphomatosis, also impede embryonic development [54]. BCFAs in chickens may have a similar function in limiting tumour cell proliferation, despite the current lack of reports on this topic. In addition, BCFAs have a close relationship with gastrointestinal health in newborn humans and are reportedly major components of a healthy gastrointestinal tract in infants [55]. In vernix, most BCFAs consist of 11-26 carbons in the anteiso-configuration. However, in meconium, BCFAs consist of 16-26 carbons in the iso-configuration [55], illustrating that active BCFA metabolism exists in the foetal GI tract and is associated with gut colonization. We supposed that Even if chickens do not have vernix, BCFAs may still play similar roles in maintaining gut microbiota stability in chicken embryos.

\subsection{Metabolic Importance of Carbohydrates in Developing Embryos}

Carbohydrates, especially glucose, have important roles on the energy related metabolism in the whole incubation period $[3,17,18,22]$. During the early incubation stage, $\mathrm{O}_{2}$ supplies are limited to what can be provided by simple diffusion with the aid of primitive haemoglobin. The energy needed for development primarily comes from the glycolysis of accessible glucose, leading to the formation of lactic acid, until chorioallantois formation occurs [3, 19]. Glucose and other carbohydrates in the egg are largely recovered from the thin outer membrane of dam during the period of uterine plumping 


\section{Chapter 2}

before shell formation $[3,56]$. However, the glucose stored in albumen and yolk is not sufficient. Thus, gluconeogenesis from other sources appears to be crucial for glucose supplementation at these times. A wide range of nutrients, such as amino acids, glycerol, fructose, dihydroxyacetone, lactate, pyruvate and fructose, impact hepatic glucose production in chickens when the supply of this carbohydrate is insufficient $[1,4,19,57]$.

From mid incubation until 2 or 3 days before internal pipping, more oxygen is supplied by the chorioallantois, and the embryo can utilize fatty acids as an important source for glucose synthesis [1, 3, 4]. Fatty acid synthesis and B-oxidation are the two main pathways for fatty acid metabolism during this stage [1, 3, 4]. In egg yolks, fatty acids are stored as triacylglycerol and phospholipids. Triacylglycerol is modified to generate free fatty acids and glycerol. Free fatty acids can be transformed into acetyl-CoA and oxidized to ATP through the TCA cycle in a reaction initially catalysed by the enzyme citrate synthase. The remaining glycerol backbone can be used to generate glucose through the gluconeogenic pathway at a rate sufficient to cover the physiological demand $[1,3$, 4]. Sunny and Bequette [6] used in ovo injections of ${ }^{13} \mathrm{C}$-labeled glycerol and observed significant enrichments of labeled ${ }^{13} \mathrm{C}$ in blood glucose and liver and muscle glycogen, which proved that glycerol is potentially a major precursor for hepatic glucose and glycogen synthesis. Gluconeogenesis only occurs in the liver and kidney of chicks, and glucose can be exported to other tissues for utilization or stored as glycogen in liver and muscle cells of chicken embryos [4]. Glycogenesis can occur via the phosphorylation of glucose and the incorporation of glucose-6-phosphate into glycogen (direct pathway) or through the incorporation of 3-carbon intermediates into glycogen via reverse glycolysis (indirect pathway) [3, 4, 20, 21]. Sunny and Bequette [6] revealed that the indirect pathway accounts for $15 \%$ of glycogen synthesis in chickens embryo, and $76 \%$ of this glycogen is derived from the direct pathway in the liver during periods of glycogenstorage in middle stage of incubation. In addition to glycerol, just before pipping, the amniotic fluid and possibly muscle proteins are other important sources of substrates for maintaining glycogen reserves in the liver and muscles [3, 4, 58]. The stored glycogen is important to supply the later pipping stage with sufficient energy until food can be consumed $[3,4,58]$. 


\section{Chapter 2}

Normally, glucose can be reduced to pyruvate and enter the TCA cycle to generate enough ATP if sufficient oxygen is present. However, in the late incubation stage, a faster way to produce energy is necessary in muscles involved in pipping because oxygen levels are not always sufficient during intensive muscle activity $[3,4,20,21]$. Therefore, pyruvate is prone to be converted to lactate $[3,4,20$, 21]. Chicken can recycle lactate back to glucose in the liver through the Cori cycle once oxygen is restored [4]. When glucose is not sufficient in the last phase, stored glycogen will be hydrolyzed to compensate plasma glucose level $[3,4,59,60]$. Embryonic stored glycogen concentrations in liver and muscle is influenced by embryonic growth rate, which is related to selection of laying performance in chicken, such as laying egg number and egg weight [59, 60, 61, 62].

\subsection{Metabolic Importance of Amino Acids in Developing Embryos}

There are initially no free amino acids in eggs, and all amino acids are present only in proteins. As the embryo develops, these proteins are gradually used to supply amino acids. As early as 3 days into the incubation, free amino acids can be identified in the yolk, and their levels increase up to day 9 [63]. In avian embryos, the yolk sac is critical for the absorption of amino acids from the yolk and albumen and is thought to play a role similar to that of the intestine in adult animals. It is generally agreed that proteins are first absorbed into yolk sac cells, particularly endodermal cells. Then they are digested by a proteinase and that the products are transported into the circulation. Cathepsin B and D have been identified in endodermal cells of quails, and their activities have been demonstrated crucial for yolk protein transport [64]. However, in chicken, only aspartic proteinase cathepsin D has been identified in the yolk sac cells [65].

Several amino acids are essential and cannot be synthesized in the embryo. A deficiency or excess of one amino acid will lead to an amino acid imbalance. Early studies have reported this phenomenon as an amino acid toxicity effect $[63,66,67,68]$. Salmon [66] researched a threonine deficiency-induced imbalance and found not only lack of threonine supplementation but also excess of methionine in the diet can result in toxicity symptom. Excessive methionine activates threonine oxidation by increasing the amount of threonine dehydratase. Subsequently, embryonic growth rate decreases. After 


\section{Chapter 2}

supplement of additional dietary threonine, toxicity effect can be alleviated. In poultry, antagonistic effects exist between many different amino acids and can depress animal growth, e.g., excess lysine antagonizes arginine and excess leucine antagonizes isoleucine and valine [67, 69, 70, 71]. Kadam et al. [14] observed that chick embryo weight tended to be lower when eggs in ovo were injected with excessive threonine, and they concluded that the higher dose could have caused an amino acid imbalance.Kermanshahi et al. [16] also found high dose in ovo injection of amino acids would impair growth of chicken embryos. Extensive studies have been conducted in rats that can serve as a basis for explaining amino acid imbalances in poultry. Yoshida et al. [72] used ${ }^{14} \mathrm{C}$-labled limiting amino acids to verify the physiological effects of amino acid imbalances in rats and found that an imbalance increase the incorporation of growth-limiting amino acids into tissues, thus reducing the levels of these essential amino acids in the plasma. Insufficient plasma amino acid levels will stimulate the body's protective response and decrease food intake.

Branched chain amino acids (BCAAs), as one group of amino acids, may have special functions for regulating protein biosynthesis in embryos. Leucine, a BCAA, has been shown to suppress protein degradation in skeletal muscles by activating protein synthesis $[73,74,75]$. In ovo injection of BCAA increased the weight of whole embryo compared to the control. Compared to the control, the in ovo administration of leucine and valine significantly accelerated the hatching time [15]. The most important proteolytic pathway is the ubiquitin-proteasome system, which is ATP-dependent and is regulated by proteasomes through the degradation of ubiquitin-conjugated proteins. BCAAs such as leucine and isoleucine can regulate ubiquitin-proteasome pathways in chicken skeletal muscles, suppressing proteolysis [76]. Leucine inhibits the expression of ubiquitin and proteasome subunits in chicken myotubes but only inhibits the expression of the proteasome $\mathrm{C} 2$ subunit in chicken skeletal muscles [76]. Isoleucine has a similar effect on the regulation of the levels of expression of proteasome $\mathrm{C} 2$ and ubiquitin subunits in chicken myotubes and skeletal muscles [76]. Nsmethylhistidine is a specific product resulting from histidine residue methylation in the myofibrillar proteins actin and myosin. Because Ns-methylhistidine cannot be utilized for protein synthesis and does not undergo catabolism, its presence in the plasma has been typically used as a marker of 


\section{Chapter 2}

myofibrillar proteolysis $[77,78]$. Feeding cultured muscle cells with leucine reduces the plasma level of Ns-methylhistidine [77, 78], indicating that leucine may have the capacity to control myofibrillar protein proteolysis. However, the mechanism underlying this control remains unclear, and the effects of other BCAAs on myofibrillar proteolysis are not understood.

\subsection{Metabolic Importance of Vitamins in Developing Embryos}

Vitamins, such as biotinand folic acid, have antitoxic effect, cell division stimulation, immune system enhancement during embryonic development in chickens in both early and late stages. Biotin and riboflavin are vitamins that have characteristics in the presence of the inhibitors avidin and ovoflavoprotein in egg albumen, which is able to generate biotin deficiencies by scavenging dietary biotin and inhibiting biotin utilizatio [79, 80], then affect their availability to the embryo [79, 81, 82]. Folic acid is a critical vitamin for cell division in all animals during reproduction, and its requirement for hatchability is higher than many other vitamins in eggs [83]. The folate content in eggs is maximized when dietary folic acid reaches $2 \mathrm{mg} / \mathrm{kg}$ as the folate precursor is saturated above this level [84]. The long-term storage of eggs leads to a folic acid deficiency [85]. Vitamin E, with strong antioxidance ability, has been found to be related to breeding efficiency in several species and to immune responses in poultry. Haq et al. [86] observed higher antibody titres in eggs after the vitamin E supplementation of breeder diets. Hens exposed to heat stress have been shown to low dietary amount and lay eggs with lower vitamin E concentrations in the yolk [87], and embryos present lower mortality rates when heat stressed hens are fed higher amounts of vitamin E compared with the mortality of embryos from hens fed the standard level [88].

Dried chicken egg whites contain avidin, which is toxic to animals [89]. However, in avian eggs or yolks, biotin neutralizes avidin and protects the embryos from damage. A deficiency of biotin during incubation is associated with a rapid increase in embryonic mortality within the first week of life, but few impacts on embryonic mortality in the last stage of incubation have been observed [12, 90, 91]. An egg biotin deficiency can be overcome by increasing dietary biotin or by injecting biotin into the egg at the beginning of incubation [12]. 


\section{Chapter 2}

The most important symptom of biotin deficiency in embryos is chondrodystrophy. In previous studies, biotin deficiencies have been shown to result in approximately half of embryos to suffer from chondrodystrophy $[91,92,93,94]$. Syndactyly also appears in biotin-deficient embryos from hens fed suboptimal amounts of biotin. Couch et al. [90] determined that injecting biotin into the egg whites of biotin-deficient eggs could increase their hatchability. The amount of biotin injected should exceed the avidin-binding capacity so that the unbound biotin can be utilized by the body, alleviating the symptoms associated with this deficiency.

\subsection{Metabolic Importance of Steroids and Hormones in Developing}

\section{Embryos}

Steroids such as cholesterol are important hormone precursors and indirectly influence embryonic development $[3,4,95,96]$. However, the pathway of steroid transport from the yolk to the embryo remains unclear. Radioactive oestradiol has been shown to be converted to polar compounds that are absorbed in large amounts by the embryo, suggesting that some bioactive enzymes serve special functions to convert steroids to water-soluble forms, which would increase efficiency in hormone and steroid transport [97]. The SULT/STS pathway may play a critical role in this process because sulfotransferase activity, which is related with this pathway, has been detected in developing chicken embryo tissues [97]. The same extra-embryonic membranes that ultimately form the placenta in mammals have been detected in oviparous vertebrates [97], and some important enzymes of the SULT/STS pathway are located in these membranes. Thus, the SULT/STS pathway is critical for animal in dealing with maternal steroid signals [98]. Deficiencies in enzymes in this pathway lead to abnormal embryonic development due to problems in the absorption of maternal steroids or hormones.

A large amount of insulin injected prior to incubation induces teratogenic changes in chick embryos during development, especially in skeletal and brain tissues [4, 99]. However, an adequate concentration of insulin is necessary in embryos for improving glucose utilization by blastoderm cells in the period from gastrulation to neurulation, when a high demand for energy must be met [100]. Insulin has a wide range of functions in stimulating glucose transport, glucose conversion to glycogen 


\section{Chapter 2}

and glucose oxidation or conversion to lactate [101]. Corresponding to an increase in glucose consumption, the insulin level in the chick blastoderm increases significantly during the processes of gastrulation and neurulation $[3,4,102]$. The observed increase in aerobic lactate production after the increase in insulin levels rather than an increase in the consumption of oxygen implicates the insulinactive cytosolic glycolytic pathway and not the mitochondrial oxidative pathway [100]. Normally, with cell division, glucose utilization via the pentose shunt increases after insulin stimulation. However, this was not observed in a study by Baroffio and Kucera [100], but this response may appear after a longer latency period.

Glucagon levels are generally low throughout incubation and only increase during the three days before hatching, peaking during pipping [2, 4]. After the chick begins to feed, glucagon levels drop by $40 \%$ in 24 hours, indicating that embryos need glucagon to accelerate glucose generation and maintain glucose levels during pipping [2, 4]. In the embryo liver, gluconeogenesis is stimulated by glucagon to produce glucose from other substrates, such as amino acids, making the sugar available to other tissues through the circulation in embryo $[4,56]$. Glucagon also plays a role during the embryonic transition from lipid to carbohydrate metabolism. As Langslow et al. [103], proposed, adipocytes become sensitive to glucagon levels only after hatching, which is important to ensure that subcutaneous adipose stores are not used until the first day post-hatch. Although the glucagon level fluctuates during incubation, glucagon may function as a complex embryonic growth-stimulating factor that interacts with other hormones [2].

Thyroid hormones, triiodothyronine (T3) and thyroxin (T4) are involved in many physiological processes, including the regulation of heat production, glycogen reserves and embryo muscle growth $[2,4,104,105,106]$. Lu et al. [2] observed constant levels of T3 in chicken embryos during midincubation that then peaked the day before hatching. T4 levels reached high levels and remained at a high plateau between 17 and 20 days of incubation in chickens and decreased after hatching. Increasing thyroxin levels are important for stimulating a variety of developmental and metabolic processes for hatching $[2,4,104,105,106]$. A sharp rise in T3 has been found to be associated with 
Chapter 2

the embryonic switch to lung respiration, and both $\mathrm{T} 3$ and $\mathrm{T} 4$ are positively correlated with embryonic body weight late in the incubation period [2].

\subsection{Conclusions}

Many metabolites impact embryonic development in poultry, and the different types of nutrient functions are summarized below:

1) Fatty acids are important energy source during incubation. In addition, some UFAs, especially omega-3 fatty acids, serve a regulatory function in embryonic development. DHA and EPA, as important omega-3 fatty acids, are highly correlated with poultry neural system development and retina.

2) Carbohydrates, mainly glucose, provide the necessary energy for the first embryonic development stage and emergence at last stage of incubation, when the oxygen level is insufficient for aerobic metabolism. Other nutritional components are converted into glucose in these stages if the stored glucose is insufficient.

3) Amino acids are the main substrate for protein synthesis and muscle growth. Among them, threonine plays a role as the third limiting amino acid and greatly influences the amino acid balance. Leucine can suppress the proteolytic pathway and prevent protein degradation.

4) Vitamins are crucial regulators that affect the prevention of abnormal embryonic development during the entire incubation period. Myo-inositol is the main source of biotin, which can greatly reduce the toxic effect of avidin in eggs and decrease the rate of foetal malformation.

5) Steroids such as cholesterol are important hormone precursors. Glucagon, insulin, thyroid hormone and other hormones have distinct but associated functions that maintain embryonic physiological stability in different situations. 


\section{Chapter 2}

\subsection{Summary}

Profits due to the application of new biotechnologies and principles of developmental biology in the poultry industry for improving the growth performance and production yield of commercial chickens have greatly improved, and this trend should continue in the future [1, 2, 3, 4]. As a crucial point in poultry production, egg incubation has been receiving more attention by researchers worldwide $[1,2$, $3,4,5,6]$. Every year, approximately $20 \%$ of eggs around the world die during incubation, which represents a great loss of biomass and economic cost in the poultry industry $[7,8,9,10,11]$. Anything that supports or limits growth and development during the incubation period will have a marked effect on overall growth performance and health of modern strains of poultry [4]. (De Oliveira et al., 2008). In past decades, tremendous efforts have focused on finding metabolites in egg yolk and albumen that impact embryonic survival ability and on determining the function of different nutritional components during distinct stages of incubation $[1,2,3,4,5,12,13,14,15,16]$. They demonstrated Fatty acids related pathways are most active during middle and late incubation stages and associated with chicken embryonic energy metabolism, neural system development, immune ability and lipiduptake. Carbohydrates are important energy source when embryonic oxygen consumption is insufficient. Essential amino acids control protein synthesis and impact chicken embryonic growth rate. Vitamins with strong anti oxidation ability could inhibit toxic effect of oxidative stress or free radicals in egg, such as avidin. Steroids such as cholesterol are important hormone precursors. The consequencesto the embryo may be lethal if the egg contains eitherinadequate, excessive, or imbalanced levels of nutrients.This article will summarize the embryonic development process and the functions of different metabolite groups in egg during the embryonic development of poultry.

\subsection{References}

1. Sato M, Tachibana T, Furuse M. Heat production and lipid metabolism in broiler and layer chickens during embryonic development. Comp Biochem Phys A 2006;143:382-388. 


\section{Chapter 2}

2. Lu JW, Mcmurtry JP, Coon CN. Developmental changes of plasma insulin, glucagon, insulin-like growth factors, thyroid hormones, and glucose concentrations in chick embryos and hatched chicks. Poultry Sci 2007;86:673-683.

3. Moran ET. Nutrition of the developing embryo and hatchling. Poultry Sci 2007;86:1043-9.

4. De Oliveira JE, Uni Z, Ferket PR. Important metabolic pathways in poultry embryos prior to hatch. World Poultry Sci J 2008;64:488-99.

5. Aydin R, Pariza MW, Cook ME. Olive oil prevents the adverse effects of dietary conjugated linoleic acid on chick hatchability and egg quality. J Nutr 2001;131:800-6.

6. Sunny NE, Bequette BJ. Glycerol is a major substrate for glucose, glycogen, and nonessential amino acid synthesis in late-term chicken embryos. J Anim Sci 2011;89: 3945-3953.

7. Beaumont C, Millet N, Le Bihan-Duval E, Kipi A, Dupuy V. Genetic parameters of survival to the different stages of embryonic death in laying hens. Poultry Sci 1997;76:1193-1196.

8. Sewalem A, Johansson K, Carlgren AB, Wilhelmson M, Lillpers K. Are reproductive traits impaired by selection for egg production in hens? J Anim Breed Genet 1998;115:281-297.

9. Dobek A, Szydlowski M, Szwaczkowski T, Skotarczak E, Molinski K. Bayesian estimates of genetic variance of fertility and hatchability under a threshold animal model. J Anim Feed Sci 2003;12: 307-314.

10. Rozempolska-Ruciska I, Zieba G, Łukaszewicz M. Hatchability traits as selection criteria in breeding of laying hens. Arch Geflugelkd 2008;73:263-267.

11. Wondmeneh E, Dawud I, Adey M. Comparative evaluation of fertility and hatchability of Horro, Fayoumi, Lohmann Silver and Potchefstroom Koekoek breeds of chicken. Asian J Poultry Sci 2011;5:124-129.

12. Wilson HR. Effects of maternal nutrition on hatchability. Poultry Sci 1997;76:134-143.

13. López-Fandiño R, Anton M, Schade R, eds. Bioactive Egg Compounds. Berlin: Springer Verlag 2007:33-66.

14. Kadam MM, Bhanja SK, Mandal AB, Thakur R, Vasan P, Bhattacharyya A, Tyagi JS. Effect of in ovo threonine supplementation on early growth, immunological responses and digestive enzyme activities in broiler chickens. Brit Poultry Sci, 2008;49:736-741. 


\section{Chapter 2}

15. Kita K, Ito RK, Sugahara M, Kobayashi M, Makino R, Takahashi N, Nakahara H, Takahashi K, Nishimukai M. Effect of in ovo administration of branched-chain amino acids on embryo growth and hatching time of chickens. J Poultry Sci 2014.

16. Kermanshahi H, Golian A, Khodambashi EN, Daneshmand A, Ghofrani TD, Ibrahim SA. Effects of in ovo injection of threonine on hatchability, intestinal morphology, and somatic attributes in Japanese quail (Coturnix japonica). J Appl Anim Res 2016;45:437-441.

17. Cirotto C, Arangi I. How do avian embryos breath? Oxygen transport in the blood of early chick embryos. Comp Biochem Physiol 1989;94: 607-613.

18. Baumann R, Meuer HJ. Blood oxygen transport in the early avian embryo. Physiol Rev 1992;72: $941-965$.

19. Kucera P, Raddatz E, Baroffio A. Oxygen and glucose uptake in the early chick embryo. Respiration and Metabolism of Embryonic Vertebrates. 1984;299-309.

20. Menna TM, Mortola JP. Metabolic control of pulmonary ventilation in the developing chick embryo. Respiratory Physiology and Neurobiology. 2002;130: 43-55.

21. Villamor E, Ruijtenbeek K, Pulgar V, De Mey JG, Blanko CE. Vascular reactivity in intrapulmonary arteries of chicken embryos during transition to ex ovo life. Am J Physiol-Reg I 2002;282: 917-927.

22. Sharifi AR, Horst P, Simianer $\mathrm{H}$. The effect of naked neck gene and ambient temperature and their interaction on reproductive traits of heavy broiler dams. Poultry Sci 2010;89:1360-1137.

23. Kuksis A. Yolk lipids. Biochim Biophys Acta 1992;1124:205-22.

24. Cherian G. Nutrition and metabolism in poultry: role of lipids in early diet. J Anim Sci Biotechnol 2015;6:1.

25. Speake BK . Metobolism and functions of omega-3 fatty acids during development of the avian embryo. In Teale MC, ed. Omega 3 Fatty Acid Research. New York, NY: Nova Science Publishers; 2006:187-223.

26. Salem JRN, Litman B, Kim HY, Gawrisch K. Mechanisms of action of docosahexaenoic acid in the nervous system. Lipids 2001;36:945-959. 


\section{Chapter 2}

27. Mitchell DC, Niu SL, Litman BJ. Enhancement of G protein-coupled signaling by DHA phospholipids. Lipids 2003;38:437-443.

28. Noble RC, Cocchi M. Lipid metabolism and the neonatal chicken. Prog Lipid Res 1990;29:107140.

29. Querques G, Forte R, Souied EH. Retina and omega-3. J Nutr Metab 2011.

30. Anderson GJ, Connor WE. On the demonstration of omega-3 essential-fatty-acid deficiency in humans. Am J Clin Nutr 1989;49: 585-587.

31. Greiner RS, Catalan JN, Moriguchi T, Salem JRN. Docosapentaenoic acid does not completely replace DHA in n-3 FA-deficient rats during early development. Lipids 2003;38: 431-435.

32. Salem N, Niebylski CD. The nervous system has an absolute molecular species requirement for proper function. Mol Membr Biol 1995;12: 131-134.

33. Cherian G. Metabolic and cardiovascular diseases in poultry: role of dietary lipids. Poultry Sci 2007;86: 1012-1016.

34. Flock MR, Harris WS, Kris-Etherton PM. Long-chain omega-3 fatty acids: time to establish a dietary reference intake. Nutr Rev 2013;71: 692-707.

35. Bautista Ortega J. Polyunsaturated fatty acid metabolism in broiler chickens: effects of maternal diet (Doctoral Dissertation). Corvallis, OR: Oregon State University. 2008.

36. Mclennan IS. A study of the capacity of various eicosanoids to stimulate skeletal muscle formation in chicken embryos. J Anat 1991;174:115.

37. Hausman RE, Berggrun DA. Prostaglandin binding does not require direct cell-cell contact during chick myogenesis in vitro. Exp Cell Res 1987;168: 457-462.

38. Nagai M. The effects of prostaglandin E1 on DNA and collagen synthesis in osteoblasts in vitro. Calcified Tissue Int 1989;44:411-420.

39. Watkins BA. Importance of essential fatty acids and their derivatives in poultry. J Nutr $1991 ; 121: 1475-85$.

40. Donaldson WE, Fites BL. Embryo mortality in quail induced by cyclopropene fatty acids: reduction by maternal diets high in unsaturated fatty acids. J Nutr 1970;100: 605-610.

41. Tullett SG. Science and the art of incubation. Poultry Sci 1990;69:1-15. 


\section{Chapter 2}

42. Leone VA, Worzalla SP, Cook ME. Evidence that maternal conjugated linoleic acid negatively affects lipid uptake in late-stage chick embryos resulting in increased embryonic mortality. Poultry Sci 2010;89:621-632.

43. Dikmen BY, Sahan U. Correlations between breeder age, egg cholesterol content, blood cholesterol level and hatchability of broiler breeders. Brit Poultry Sci 2007;48:98-103.

44. Rumsey SC, Galeano NF, Lipschitz L, Deckelbaum RJ. Oleate and other long chain fatty acids stimulate low density lipoprotein receptor activity by enhancing acyl coenzyme A: cholesterol acyltransferase activity and altering intracellular regulatory cholesterol pools in cultured cells. $\mathrm{J}$ Biol Chem 1995;270:10008-16.

45. Brynes AE, Edwards CM, Jadhav A, Ghatei MA, Bloom SR, Frost GS. Diet-induced change in fatty acid composition of plasma triacylglycerols is not associated with change in glucagon-like peptide 1 or insulin sensitivity in people with type 2 diabetes. Am J Clin Nutr 2000;72:1111-1118.

46. Vessby B, Uusitupa M, Hermansen K, Riccardi G, Rivellese AA, Tapsell LC, Calvert GD. Substituting dietary saturated for monounsaturated fat impairs insulin sensitivity in healthy men and women: The KANWU Study. Diabetologia 2001;44:312-319.

47. Fukuchi S, Hamaguchi K, Seike M, Himeno K, Sakata T, Yoshimatsu H. Role of fatty acid composition in the development of metabolic disorders in sucrose-induced obese rats. Exp Biol Med (Maywood) 2004;229:486-93.

48. Hofman FM, Von Hanwehr RI, Dinarello CA, Mizel SB, Hinton D, Merrill JE. Immunoregulatory molecules and IL-2 receptors identified in multiple sclerosis brain. J Immunol 1986;136: 32393245 .

49. Moynagh PN. The interleukin-1 signalling pathway in astrocytes: a key contributor to inflammation in the brain. J Anat 2005;207:265-269.

50. Body DR, Reid B. The lipid and amino acid composition of the kiwi (Apteryx australis mantelli) ratite eggs. J Sci Food Agric 1983;34:587-92.

51. Yang Z, Liu S, Chen X, Chen H, Huang M, Zheng J. Induction of apoptotic cell death and in vivo growth inhibition of human cancer cells by a saturated branched-chain fatty acid, 13methyltetradecanoic acid. Cancer Res 2000;60:505-9. 
52. Wongtangtintharn S, Oku H, Iwasaki H, TODA T. Effect of branched-chain fatty acids on fatty acid biosynthesis of human breast cancer cells. J Nutr Sci Vitaminol 2004;50:137-43.

53. Wongtangtintharn S, Oku H, Iwasaki H, Inafuku M, Toda T, Yanagita T. Incorporation of branched-chain fatty acid into cellular lipids and caspase-independent apoptosis in human breast cancer cell line, SKBR-3. Lipids Health Dis 2005;4:1.

54. Sutton JB. Tumours in animals. J Anat Physiol 1885;19:414.1-475.

55. Ran-Ressler RR, Devapatla S, Lawrence P, Brenna, JT. Branched chain fatty acids are constituents of the normal healthy newborn gastrointestinal tract. Pediatr Res 2008;64:605-9.

56. De Oliveira JE. Effects of in ovo feeding on turkey embryos development, energy status, intestinal maturation, gene expression and post-hatch development. ProQuest 2007.

57. Dickson AJ, Langslow DR. Hepatic gluconeogenesis in chickens. Mol Cell Biochem 1978;22:167-182.

58. Uni Z, Ferket PR, Tako E, Kedar O. In ovo feeding improves energy status of late-term chicken embryos. Poultry Sci 2005;84:764-770.

59. Christensen VL, Donaldson WE, Nestor KE. Effect of supplemental oxygen on blood plasma organic acids within embryos from selected lines of turkeys. Poultry Sci 1999;78:1601-5.

60. Christensen VL, Donaldson WE, Nestor KE, McMurtry JP. Effect of genetics and maternal dietary iodide supplementation on turkey embryonic growth. Poultry Sci 1999;78:883-889.

61. Christensen VL, Donaldson WE, Nestor KE. Embryonic viability and metabolism in turkey lines selected for egg production or growth. Poultry Sci 1993;72:829-838.

62. Christensen VL, Nestor KE. Changes in functional qualities of turkey eggshells in strains selected for increased egg production or growth. Poultry Sci 1994;73:1458-1464.

63. Williams MA, Dacosta WA, Newman LH, Marshall LM. Free amino-acids in the yolk during the development of the chick. Nature 1954;173:490.

64. Gerhartz B, Kolb HJ, Wittmann J. Proteolytic activity in the yolk sac membrane of quail eggs. Comparative Biochemistry and Physiology Part A: Molecular and Integrative Physiology $1999 ; 123: 1-8$ 


\section{Chapter 2}

65. Wouters J, Goethals M, Stockx J. Acid proteases from the yolk and the yolk-sac of the hen's egg. Purification, properties and identification as cathepsin D. Int J Biochem 1985;17:405-413.

66. Salmon WD. The significance of amino acid imbalance in nutrition. Am J Clin Nutr 1958;6:437494.

67. D'mello JPF, Lewis D. Amino acid interactions in chick nutrition: I. The interrelationship between lysine and arginine. Brit Poultry Sci 1970;11:299-311.

68. Park BC. Amino acid imbalance-biochemical mechanism and nutritional aspects. Asian Australas J Anim Sci 2006;19:1361.

69. Kidd MT, Kerr BJ. L-threonine for poultry: A review. J Appl Poultry Res 1996;5:358-367.

70. Meister A. Biochemistry of The Amino Acids. Elsevier. 2012.

71. Wecke C, Liebert F. Improving the reliability of optimal in-feed amino acid ratios based on individual amino acid efficiency data from N balance studies in growing chicken. Animal 2013;3: $558-573$.

72. Yoshida A, Leung PM, Rogers QR, Harper AE. Effect of amino acid imbalance on the fate of the limiting amino acid. J Nutr 1966;89:80-90.

73. Anthony JC, Yoshizawa F, Anthony TG, Vary TC, Jefferson, LS, Kimball SR. Leucine stimulates translation initiation in skeletal muscle of postabsorptive rats via a rapamycin-sensitive pathway. $\mathbf{J}$ Nutr 2000;130:2413-2419.

74. Yoshizawa F, Hirayama S, Sekizawa H, Nagasawa T, Sugahara K. Oral administration of leucine stimulates phosphorylation of 4E-bP1 and S6K 1 in skeletal muscle but not in liver of diabetic rats. J Nutr Sci Vitaminol 2002;48:59-64.

75. Yoshizawa F. Regulation of protein synthesis by branched-chain amino acids in vivo. Biochem Bioph Res Co 2004;313:417-422.

76. Nakashima K, Ishida A, Yamazaki M, And Abe H. Leucine suppresses myofibrillar proteolysis by down-regulating ubiquitin-proteasome pathway in chick skeletal muscles. Biochem Bioph Res Co 2005;336:660-666. 


\section{Chapter 2}

77. Nagasawa T, Hirano J, Yoshizawa F, Nishizawa N. Myofibrillar protein catabolism is rapidly suppressed following protein feeding. Bioscience, Biotechnology and Biochemistry. 1998;62: 1932-1937.

78. Nagasawa T, Kido T, Yoshizawa F, Ito Y, Nishizawa N. Rapid suppression of protein degradation in skeletal muscle after oral feeding of leucine in rats. J Nutr Biochem 2002;13:121-127.

79. Donovan JW, Ross KD. Increase in the stability of avidin produced by binding of biotin. Differential scanning calorimetric study of denaturation by heat. Biochemistry 1973;12:512-517.

80. Hytönen VP, Nyholm TK, Pentikäinen OT, Vaarno J, Porkka EJ, Nordlund HR, Kulomaa MS. Chicken avidin-related protein $4 / 5$ shows superior thermal stability when compared with avidin while retaining high affinity to biotin. J Biol Chem 2004;279: 9337-9343.

81. Kratzer FH, Knollman K, Earl L, Buenrostro JL. Availability to chicks of biotin from dried egg products. J Nutr 1988;118:604-608.

82. Vieira SL. Chicken embryo utilization of egg micronutrients. Revista Brasileira de Ciência Avícola 2007;9:1-8.

83. Taylor LW. The effect of folic acid on egg production and hatchability. Poultry Sci 1947;26:372376.

84. Hebert K, House JD, Guenter W. Effect of dietary folic acid supplementation on egg folate content and the performance and folate status of two strains of laying hens. Poultry Sci 2005;84:15331538.

85. White H, Whitehead CC. Role of avidin and other biotin-binding proteins in the deposition and distribution of biotin in chicken eggs. Discovery of a new biotin-binding protein. Biochem J 1987;241:677-684.

86. Haq AU, Bailey CA, Chinnah AD. Neonatal immune response and growth performance of chicks hatched from single comb white leghorn breeders fed diets supplemented with $\beta$-carotene, canthaxanthin, or lutein. Poultry Sci 1995;74: 844-851.

87. Kirunda DF, Scheideler SE. The efficacy of vitamin E (DL-alpha-tocopheryl acetate) supplementation in hen diets to alleviate egg quality deterioration associated with high temperature exposure. Poultry Sci 2001;80:1378-1383. 
88. Siegel PB, Blair M, Gross WB, Meldrum B, Larsen C, Boa-Amponsem K, Emmerson DA. Poultry performance as influenced by age of dam, genetic line, and dietary vitamin E. Poultry Sci 2006;85:939-942.

89. Eakin RE, Mckinley WA, Williams RJ. Egg-white injury in chicks and its relationship to a deficiency of vitamin $\mathrm{H}$ (biotin). American Association for the Advancement of Science. Science 1940;92: 224-225.

90. Couch JR, Cravens WW, Elvehjem CA, Halpin JG. Relation of biotin to congenital deformities in the chick. Anat Record 1948;100:29-48.

91. Taniguchi A, Watanabe T. Roles of biotin in growing ovarian follicles and embryonic development in domestic fowl. J Nutr Sci Vitaminol 2007;53:457-463.

92. Dunn LC. The occurrence of chondrodystrophy in chick embryos II. The genetic evidence. Dev Genes Evol 1927;110: 341-365.

93. Byerly TC, Titus HW, Ellis NR. Effect of diet on egg composition II. Mortality of embryos in eggs from hens on diets containing protein supplements of different origin. J Nutr 1933;6: 225242.

94. Lyons M, Insko WM. Chondrodystrophy in the chick embryo produced by manganese deficiency in the diet of the hen. 1937.

95. Elf PK, Fivizzani AJ. Changes in sex steroid levels in yolks of the leghorn chicken, Gallus domesticus, during embryonic development. J Exp Zool 2002;293: 594-600.

96. Von Engelhardt N, Henriksen R, Groothuis TG. Steroids in chicken egg yolk: metabolism and uptake during early embryonic development. Gen Comp Endocr 2009;163:175-183.

97. Paitz RT, Bowden RM. A proposed role of the sulfotransferase/sulfatase pathway in modulating yolk steroid effects. Integr Comp Biol 2008;48:419-427.

98. Levitz M. Conjugation and transfer of fetal-placental steroid hormones 1. J Clin Endocr Metab 1966;26:773-777.

99. Tokushima Y, Takahashi K, Sato K, Akiba Y. Glucose uptake in vivo in skeletal muscles of insulin-injected chicks. Comp Biochem Phys B 2005;141:43-48. 


\section{Chapter 2}

100. Baroffio A, Kucera P. The deoxyglucose method adapted for studies of glucose metabolism in the early chick embryo. J Cell Physiol 1985;123:111-116.

101. Crettaz M, Prentki M, Zaninetti D, Jeanrenaud B. Insulin resistance in soleus muscle from obese zucker rats. Involvement of several defective sites. Biochem J 1980;186: 525-534.

102. Patwardhan V, Gokhale M, Ghaskadbi S. Acceleration of early chick embryo morphogenesis by insulin is associated with altered expression of embryonic genes. Int J Dev Biol 2004;48:319-326.

103. Langslow DR, Cramb G, Siddle K. Possible mechanisms for the increased sensitivity to glucagon and catecholamines of chicken adipose tissue during hatching. Gen Comp Endocr 1979;39: 527533.

104. Christensen VL, Donaldson WE, McMurtry JP. Physiological differences in late embryos from turkey breeders at different ages. Poultry Sci 1996;75:172-178.

105. Decuypere E, Willemsen H. Physiological control mechanisms during late embryogenesis and during pipping and hatching. Post Graduation Course in Incubation Biology and Management. Univ of Wageningen Holland 2006.

106. De Groef B, Grommen SV, Darras VM. Hatching the cleidoic egg: the role of thyroid hormones. Front Endocrinol 2013:4:63. 


\title{
CHAPTER 3 STATISTICAL AND GENETIC
}

ANALYSIS OF HATCHABILITY TRAITS IN LAYING

\author{
HENS
}


Chapter 3

\section{Statistical and Genetic Analysis of Hatchability Traits in Laying Hens}

\subsection{Introduction}

In commercial egg production, hatching traits are important in breeding programmes which have significant economic implications for franchise hatcheries due to their higher demand for day-old chicks [1]. In the chicken industry, hatchability rates range from $70 \%$ to $85 \%[2,3,4,5,6]$. Taking into consideration that more than 60 billion day-old layers and broiler chicks are produced worldwide annually [7], a marginal improvement in hatchability would generate enormous savings in biomass, energy cost and waste disposal [8]. To minimize these losses, the need to improve embryonic resistance to mortality by optimising environmental conditions and improving genetic factors appears to be increasingly urgent. The high reproductive capacity of chickens and low generation interval leads to higher genetic progress of desirable traits in the nucleus tie and accelerates the transfer of genetic improvements from the nucleus into the production tie [9].

The phases of embryonic mortality are associated with major changes in the embryonic anatomical, physiological and metabolic parameters that may be affected by suboptimal environmental conditions and genetics influencing embryo survival ability. During the first week of incubation, oxygen supply is limited due to the embryo's underdeveloped chorionic vascular system. This means the energy for embryonic development relies on the anaerobic glycolysis of glucose and other carbohydrates. From day 8 to approximately the day 17 , the chorioallantois is fully developed so that adequate $\mathrm{O}_{2}-\mathrm{CO}_{2}$ gas exchange is secured [10]. This embryonic stage is characterized by rapid growth of the embryo and its completion [10]. In the last stage of development, starting from day 18 of incubation, the embryos undergo dramatic metabolic changes and physiological events such as the start of pulmonary ventilation, oral consumption of amnion, external pipping and hatching from the shell [10]. Since major physiological and metabolic changes occur during this stage of development, any environmental disturbance during this phase or improper metabolism activities during previous periods will affect embryonic viability or influence post-hatching performance [10]. As a fitness trait, embryonic viability is associated with a series of environmental factors acting in different aforementioned phases of 


\section{Chapter 3}

embryonic development, such as embryonic nutrition status influenced by maternal investment $[11,12$, 13]; egg contamination by germs [14]; incubation and pre-incubation conditions such as preincubation storage length [15]; incubator temperature; humidity; carbon dioxide concentration; gas exchange; hen age [16] and frequency of turning during incubation [17, 18, 19]. Apart from these factors, the genetics of the embryo play an important role in the embryonic survival ability. It has been established that polygenic factors, as well as single lethal genes and chromosomal aberrations, lead to malformations, malpositions and metabolic disorders during different stages of embryonic development and consequently to embryonic death [13]. Inbreeding depression caused by the cumulative effects of deleterious mutations is reported to decrease fertility and hatchability; a problem that is relevant in parental breeding lines [20,21, 22, 23]. Due to the aforementioned physiological and genetic factors influencing embryonic survival ability at different stages of incubation, the frequency of embryonic death is not equally distributed over the course of incubation. In fact, three distinct stages can be identified: early embryonic survival ability (EES) occurs during the first week of incubation; middle embryonic survival ability (MES) occurs after the first week of incubation and before transfer of the eggs into hatchery; and late embryonic survival ability (LES) occurs between day 18 of incubation and hatching [2]. Embryonic mortality is highest during the first and the third phase [24]. The main goal of this study is to analyse the components of genetic variation for fertility (FER), hatchability of all egg sets (HTE) hatchability of fertilized eggs (HFE), early (EES), middle (MES), late embryonic mortalities (LES), external pipping ability (EXP) and internal pipping ability (INP). Analysis is conducted by opening a large number of dead hatching eggs from different breeding lines (based on the precisely determined time of mortality) for data collection.

\subsection{Material and Methods}

\subsubsection{Animals}

Two independent experiments were designed. In the first experiment, hatching eggs were collected from two fully pedigreed, random mating, experimental lines consisting of 99 white and 100 brown laying hens (Friedrich-Loeffler-Institut, Germany). In the second experiment, hatching eggs were 


\section{Chapter 3}

collected from two fully pedigreed, selected commercial (Lohmann Company, germany) lines consisting of 2,909 brown (BLC) and 3,319 white laying hens (WLC). In total, 42,688 eggs from commercial white laying hens, 38,228 eggs from commercial brown laying hens, 2,903 eggs from experimental white laying hens (WLE) and 3,080 eggs from experimental brown laying hens (BLE) were used. All of the experiments were carried out at Lohmann Company, the Friedrich-LoefflerInstitut and the University of Goettingen in Germany.

\subsubsection{Data and Measurement}

To analyse fertility and hatchability traits, the commercial flock hens were artificially inseminated weekly with $0.4 \mathrm{~mL}$ of mixed and diluted semen from at least 10 roosters in order to overcome the effect of individual males on reproduction traits. Due to the limited number of male of experimental flock, the hens were artificially inseminated with semen from 3 roosters. Hatching eggs were sampled from the 43 rd to the 46 th week of age inclusive. The eggs were collected 3 times a day and incubated at $16^{\circ} \mathrm{C}$ and $60-70 \% \mathrm{HR}$ after a maximum storage time of 15 days. Cracked or misshapen eggs were removed. The eggs were subsequently incubated for 524 hours. The eggs were candled on week 7 and 18 of incubation. Eggs not exhibiting a living embryo at candling, and eggs that had not hatched by the end of the incubation time, were opened and examined macroscopically to assess the true fertility and to estimate the time of embryonic death using the Hamburger and Hamilton method [25]. One factor that contributes to high mortality rates is the incidence of embryo malpositions during the last stage of embryonic development [26]. At the end of incubation, the chicken embryo moves itself to a normal position so that the long axis of the body is parallel to the long axis of the egg, with the head towards the blunt end. The head is turned to the right and lies under the right wing, and the tip of the beak points towards the air cell. However, should deviation from this normal position occur, there are several unusual embryonic positions which may hinder or prevent the embryo from hatching $[12,27]$. These positions are termed malposition. Previous studies divide these malpositions into seven categories [12, 27, 28]: I. Head between thighs. II. Head in small end of the egg; III. Head under left wing instead of right; IV. Head in normal position but rotated with beak pointed away from air cell; V. Feet over head; VI. Head over right wing; VII. Embryo is placed crosswise to the long axis of egg. 


\section{Chapter 3}

Based on the product-limit survival analysis for embryonic survival ability performed in this study, and in agreement with previous studies [2, 29], the embryonic mortality for different time periods of embryonic development was considered as follows: early embryonic mortality occurs during the first week of incubation; middle embryonic mortality occurs after week 1 of incubation and before the eggs are transferred into the hatcher; and late embryonic mortality occurs between day 18 of incubation and hatching time. FER, HTE, HFE, EES, MES, LES, EXP and INP were determined for each hen individually and described in detail as follows: FER is the proportion of the total number of fertilized eggs to the total number of incubated eggs. HTE is the proportion of the total number of hatched eggs to the total number of incubated eggs. HFE is the proportion of the total number of hatched egg to the total number of fertilized eggs. EES is the proportion of survival embryos during early embryonic development to the total number of fertilized eggs. MES is the proportion of survival embryos during middle embryonic development to the total number of embryos that survive the early stage. LES equates to the late stage survival egg number divided by the middle stage survival egg number. EXP represents the external pipped egg number divided by the middle stage survival egg number. INP equals the internal pipped egg number divided by the middle stage survival egg number.

\subsubsection{Statistical and Genetic Data Analysis}

The survival curves for embryonic survival during the course of incubation are produced by KaplanMeier analysis in SAS [30] using the following flow model:

$$
\hat{S}(t)=\prod_{j: t_{j} \leq t}\left[1-\frac{d_{j}}{n_{j}}\right] ; \text { for } t_{1} \leq t \leq t_{k}
$$

Where $\hat{S}(t)$ is the survival function, $n_{j}$ is the number of embryos at risk of embryonic mortality, and $d_{j}$ is the number of embryos that do not survive at time $t_{j}$.

Statistical and genetic analysis of fertility, embryonic mortality and hatchability data was performed using univariate generalized linear mixed models with repeated measurements in ASREML [31]. The statistical model for the commercial line is presented below: 
Chapter 3

$$
\log \left(\pi_{r s t u v w} /\left(1-\pi_{\text {rstuvw }}\right)\right)=\varphi+\alpha_{r}+\beta_{s}+\eta_{t}+\lambda_{u}+\alpha_{r} * \beta_{s}+\alpha_{r} * \eta_{t}+\alpha_{r} * \lambda_{u}+\gamma_{v}+\rho_{w}
$$

Where $\pi$ is the probability of hatchability of all egg set eggs, the probability of hatchability for fertile eggs, or the probability of EES, MES and LES; $\varphi$ is the overall mean effect; $\alpha$ is the fixed effect of poultry houses; $\beta$ is the fixed effect of side; $\eta$ is the fixed effect of floor; $\lambda$ is the fixed effect of hatch; $\gamma$ is the random genetic effect of animals and $\rho$ is the randomness of permanent environmental effects on animals.

The environmental condition in which FLI lines are housed differs slightly from that of the Lohmann commercial lines. Thus, another statistical model for FLI lines was used:

$$
\log \left(\pi_{r s t u v} /\left(1-\pi_{r s t u v}\right)\right)=\varphi+\beta_{r}+\eta_{s}+\lambda_{t}+\beta_{r} * \eta_{s}+\beta_{r} * \lambda_{t}+\gamma_{u}+\rho_{v}
$$

Where $\pi$ is the probability of hatchability in all eggs, the probability of hatchability for fertile eggs, or the probability of EES, MES and LES; $\varphi$ is the overall mean effect; $\beta$ is the fixed effect of side; $\eta$ is the fixed effect of floor; $\lambda$ is the fixed effect of hatch; $\gamma$ is the random genetic effect of animals and $\rho$ is the random effect of permanent environment. Heritabilities were calculated using the variance of the logit link function. This implies a correction of the residual variance by factor $\pi^{2} / 3$ presented as follows:

$$
h^{2}=\frac{\sigma_{A}^{2}}{\sigma_{A}^{2}+\sigma_{E P}^{2}+\pi^{2} / 3}
$$

Where $\sigma_{A}^{2}$ is the addictive genetic variance and $\sigma_{E P}^{2} \mathrm{~s}$ the variance of permanent environment. Estimates of random effects of animal (EBV) were calculated in a univariate model. The estimates of random effects of animals (EBV) on the logit scale were back-transformed to the original scale using the inverse link function $\mathrm{BV}=\exp (\mu+\mathrm{u}) /[1+\exp (\mu+\mathrm{u})]$. Genetic correlations between different reproductive traits were estimated using multivariate linear approaches based on the aforementioned statistical models, as applied elsewhere [32]. 


\section{Chapter 3}

\subsection{Results and Discussion}

As shown in Table 3.1, the fertility rates of the lines studied were similar and within the range $94 \%$ to 97\%. Fertility rates were $94.9 \%$ in BLC, $97.1 \%$ in WLC, $95.2 \%$ in BLE and $96.4 \%$ in WLE. These results are similar to those of the white laying population reported by Alsobayel and Albadry [33] (91\%), but were generally higher than those noted by Wondmeneh et al. [6] (86\%). Fertilization is a complex physiological process in chickens, involving spermatogenesis and sperm quality; sperm deposited at copulation; sperm transfer to the uterovaginal sperm storage tubules; sperm transfer to the site of fertilization in infundibulums, as well as the binding to and penetration of the perivetelline membrane [34]. Besides the genetic factors, there are various environmental factors, which impact the aforementioned fertilization process such as health, nutrition, temperature and light conditions of sire and dams. Previous studies carried out on laying populations have shown that due to a negative relationship between laying ability and fertility, fertilization is affected by intensive selections for egg number, egg weight and egg mass $[3,5,35]$. Compared with selection for egg number and egg mass, selection for increased egg weight has a great impact upon fertility decline over generations [3]. However, in this study, there are barely any differences between selected commercial lines and unselected experimental lines irrespective of whether brown lines (BLC, 94.9\%; BLE, 95.2\%) or white lines (WLC, 97.1\%\%; WLE, 96.4\%) are being considered.

Figure 3.1 illustrates the trend changes of embryonic survival ability during incubation. Approximately $8 \%$ suffered from embryonic death in the first 5 days, after which point the diminishing in survival rate gradually decreases until day 6 or 7 . During the period from day 7 to 17 of incubation, the survival rates for all lines remain almost unchanged. However, after day 17 of incubation, survival rates dramatically decrease until the last day of incubation. These findings reflect the trend that was also confirmed by former pieces of research $[24,27,36]$; namely that the majority of embryos die in the early and the late stages of incubation, and these represent the most critical phases for embryonic survival. 
Table 3.1:Reproduction performance for fertility (FER), hatchability of all eggs (HTE), hatchability of fertile eggs (HFE), early stage survival ability (EES), middle stage survival ability (MES), late stage survival ability (LES), external pipping ability (EXP) and internal pipping ability (INP), differences between late survival rates and internal pipping (DIL) and survival rates and external pipping (DEL).

\begin{tabular}{|c|c|c|c|c|c|c|c|c|c|c|}
\hline \multirow{2}{*}{ Line } & \multicolumn{8}{|c|}{ Embryonic development stages $^{1}$} & \multirow[b]{2}{*}{ DIL } & \multirow[b]{2}{*}{ DEL } \\
\hline & FER & HTE & HFE & EES & MES & LES & EXP & INP & & \\
\hline $\begin{array}{l}\text { Commercial } \\
\text { Brown Line }\end{array}$ & 94.9 & 72.0 & 75.3 & 90.5 & 97.3 & 84.1 & 86.9 & 90.4 & 6.3 & 2.8 \\
\hline $\begin{array}{l}\text { Commercial White } \\
\text { Line }\end{array}$ & 97.1 & 72.2 & 74.1 & 91.4 & 97.2 & 82.7 & 90.4 & 92.0 & 9.3 & 7.7 \\
\hline $\begin{array}{l}\text { Experimental } \\
\text { Brown Line }\end{array}$ & 95.2 & 78.6 & 81.8 & 89.3 & 98.5 & 92.4 & 93.8 & 96.0 & 3.6 & 1.4 \\
\hline $\begin{array}{l}\text { Experimental white } \\
\text { Line }\end{array}$ & 96.4 & 73.0 & 75.5 & 90.2 & 95.6 & 85.5 & 88.2 & 89.4 & 3.9 & 2.7 \\
\hline
\end{tabular}

${ }^{1}$ The values are the proportions of different reproduction traits.

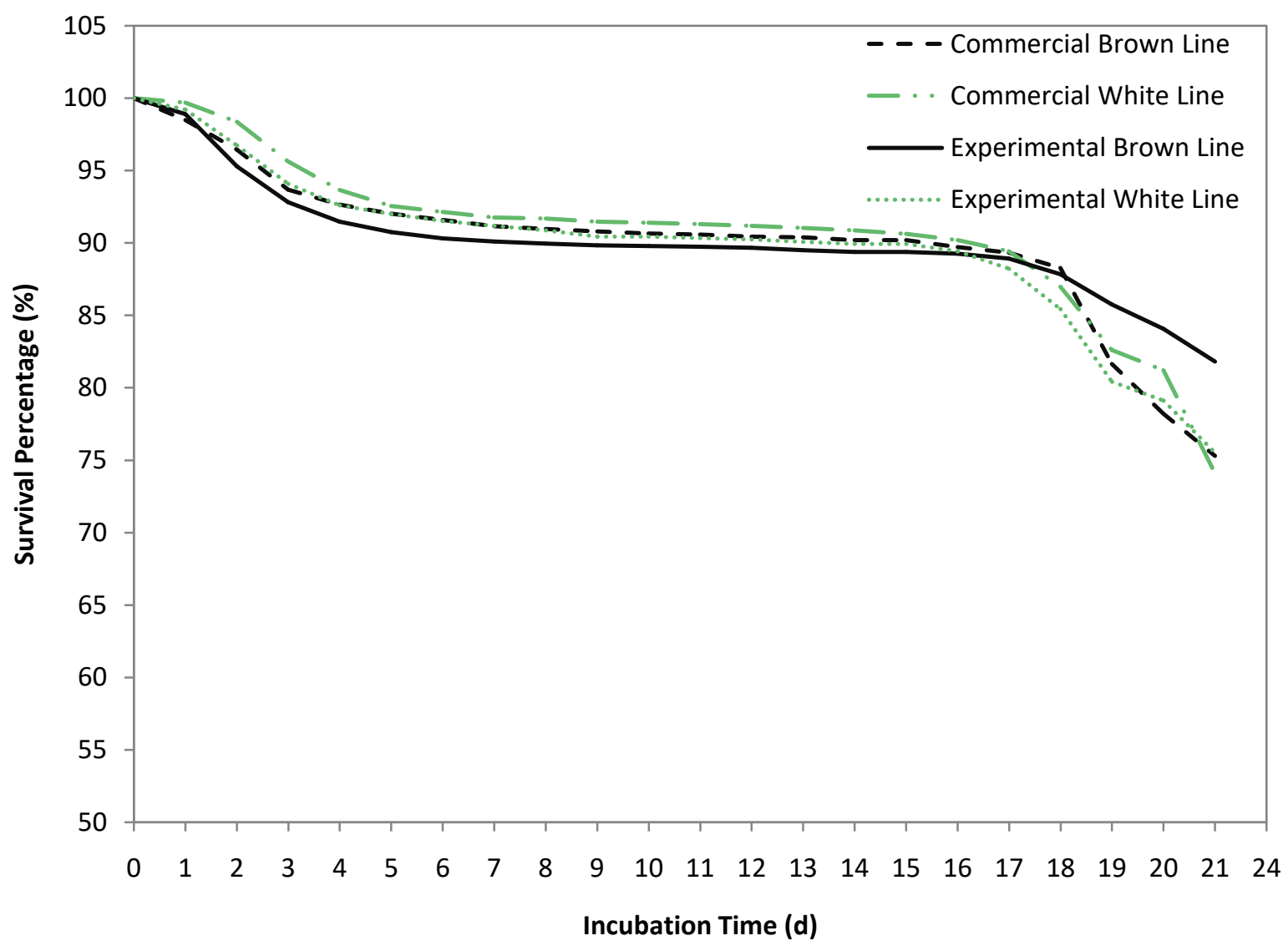

Figure 3.1:Chicken embryonic survival curves during incubation in the Commercial Brown Line, the Commercial White Line, the Experimental Brown Line and the Experimental White Line. The curves are based on product limit estimation. 


\section{Chapter 3}

The early embryonic survival rates in fertilized eggs in lines fluctuated around the $90 \%$ mark, and equated to $90.5 \%$ for BLC, $91.4 \%$ for WLC, $89.3 \%$ for BLE and $90.2 \%$ for WLE (Table 3.1). The results of this study are in agreement with Larivière et al. [29], Kuurman et al. [36] and Kuurman et al. [24], who reported an early embryonic survival ability of $90 \%$. Moran [10] summarized the first stage to include the first week of incubation, in which the egg compartments, including the amnion, chorion, allantois and yolk sac, are formed. In this phase of development, the oxygen supply is insufficient due to the immature blood cells and the underdeveloped chorionic vascular system. Thus, the energy for embryonic development relies on the anaerobic glycolysis of glucose and other carbohydrates. Several factors affecting embryonic survival ability during this stage of embryonic development such as genetics, age of hens, time of oviposition, egg weight, and quality are described and summarized by Christensen [19] and Sharifi et al. [37]. During this stage, embryos are extremely sensitive to their incubation environment, such as the incubation temperature and relative humidity. In general, a suboptimal high incubation temperature leads to embryonic hyperthermia, resulting in suppression of the embryonic growth rate and a rise in the incidence of embryonic malformation and subsequently embryonic death $[19,28]$. The optimum temperature varies depending on air humidity. Relative humidity has a significant influence on embryonic development and viability due to embryonic gas exchange and the degree of desiccation $[19,28]$. The embryonic development stage at oviposition time was found to have a notable effect on early embryonic viability. First and last eggs in clutch are believed to affect the early embryonic survival rate because they spend longer in the oviduct at an advanced embryonic developmental stage $[15,38]$. Other research work could not confirm the strong association between oviposition time or clutch position and early embryonic survival [39, 40]. In addition, selection for low body weight compared to selection for high body weight resulted in eggs with superior embryonic development at ovipositon and improved survival rates [19, 41]. However, the physiologic process is still not clear, and further research into the effect of body weight on early embryonic development is recommended. 


\section{Chapter 3}

Table 3.2: Estimation of heritabilities for fertility (FER), hatchability of all eggs (HTE), hatchability of fertile eggs (HFE), early stage survival ability (EES), middle stage survival ability (MES), late stage survival ability (LES), external pipping ability (EXP) and internal pipping ability (INP).

\begin{tabular}{lllllllll}
\hline \multirow{2}{*}{ Line } & \multicolumn{7}{c}{ Embryonic development stages } \\
\cline { 2 - 9 } & FER & HTE & HFE & EES & MES & LES & EXP & INP \\
\hline Commercial Brown & $0.095 \pm$ & $0.044 \pm$ & $0.050 \pm$ & $0.029 \pm$ & $0.012 \pm$ & $0.089 \pm$ & $0.083 \pm$ & $0.102 \pm$ \\
Line & 0.015 & 0.011 & 0.013 & 0.011 & 0.018 & 0.019 & 0.012 & 0.014 \\
Commercial White & $0.129 \pm$ & $0.052 \pm$ & $0.051 \pm$ & $0.045 \pm$ & $0.030 \pm$ & $0.060 \pm$ & $0.047 \pm$ & $0.058 \pm$ \\
Line & 0.009 & 0.010 & 0.015 & 0.012 & 0.018 & 0.012 & 0.011 & 0.013 \\
Experimental Brown & $0.105 \pm$ & $0.079 \pm$ & $0.056 \pm$ & $0.103 \pm$ & $0.188 \pm$ & $0.017 \pm$ & & \\
Line & 0.045 & 0.067 & 0.065 & 0.089 & 0.109 & 0.053 & 0.001 & $<0.001$ \\
Experimental White & $0.125 \pm$ & $0.052 \pm$ & $0.060 \pm$ & $0.045 \pm$ & $0.146 \pm$ & $0.060 \pm$ & $0.082 \pm$ & $0.105 \pm$ \\
Line & 0.013 & 0.010 & 0.016 & 0.012 & 0.057 & 0.012 & 0.012 & 0.011 \\
\hline
\end{tabular}

From a breeding point of view, brown layers are heavier than white layers. However, when comparing brown layers with white layers in terms of EES, the differences between these two types are almost negligible. It is reasonable to expect that low performing layer lines (experimental lines vs. commercial lines) exhibit a smaller clutch-size, larger time interval between two ovipositioned eggs and more eggs are generally laid in the afternoon $[19,38]$. However in this context also, no difference was found in EES between the high performing line compared with its low performing counterpart (BLC: 90.5\% vs. BLE: 89.3\%; WLC: 91.4\% vs. WLE: 90.2\%). Jaap and Fechheimer [42] highlighted hormonal changes with aging hens as a potential critical factor for the development of chromosomal abnormalities in chicken embryos. Previous studies have shown that chromosomal abnormalities can lead to significant (1 to 12\%) loss during early embryonic development. These chromosomal abnormalities are mainly numerical alterations, such as triploidy, haploidy, polyploidy, which occur during meiosis or fertilization as well as at the beginning of embryonic development [13]. Besides early embryonic development, chromosomal abnormalities may impact other stages of development and result in great losses to total hatchability $[13,43]$. In general, chromosomal abnormalities vary amongst different breeds, families and individuals. It seems that heavyweight lines are more affected than in the group of less weight [13]. 
Middle survival rates were BLC 97.3\%, WLC 97.2\%, BLE 98.5\% and WLE 95.6\% (Table 3.1). Former pieces of research also proved middle embryonic mortality to be approximately $5 \%[24,27,29$, 36]. Only a small proportion of embryonic death incidents occurred in the middle stage. During this stage, an increased amount of nutrients was absorbed for embryogenesis. Moran [10] stated that nutritional deficiencies in hens during egg formation can lead to inadequate nutrient supplements for middle embryonic development, and embryonic deaths can result during this interval. Furthermore, improper incubation procedures result in increased MEM.

The most critical period for embryonic survival is the late phase. This starts on day 18 of incubation, and has been proven to be the second peak of embryonic mortality [27]. The late stage survival rates, determined in the present study, were BLC $84.1 \%$, WLC $82.7 \%$, BLE $92.4 \%$ and WLE $85.5 \%$. These results are in agreement with former pieces of research, which indicated that late embryonic mortality was approximately $20 \%[24,27,29,36]$. The internal pipping rates were BLC $90.4 \%$, WLC $92.0 \%$, BLE 96.0\%, and WLE 89.4\%. The respective external pipping rates were BLC 86.9\%, WLC 90.4\%, BLE $93.8 \%$ and WLE $88.2 \%$. The late embryonic developmental stage coincides with the increasing demand for oxygen and a series of physiological events such as initiation of pulmonary ventilation, external pipping, and hatching from the shell. The embryonic oxygen uptake starts once chorioallantois has been fully developed [37, 44, 45]. It then becomes exponential and reaches a plateau before 3 days of internal pipping [46]. However, due to the limited eggshell and membranes for gas exchange, the embryos experience hypoxia and hypercapnia during this time. Following plateau stage, the paranatal stage is characterized by penetration of the peak in the air cell (internal pipping), external pipping and the onset of pulmonary respiration [37]. The paranatal stage may also be affected by residual effects of the plateau in oxygen uptake. During the late embryonic development, anaerobic glycogenolysis is a major source of energy [47, 48]. In this study, the late embryonic survival ability of selected lines is lower than that of experimental lines, and within these groups, the white layer are more affected by late death than the brown layers (BLC: $84.1 \%$ vs. BLE: 92.4\%; WLC: $82.7 \%$ vs. WLE: $85.5 \%$ ). When considering differences between late survival rates and internal pipping (DIL), and survival rates and external pipping (DEL), it became apparent that more 


\section{Chapter 3}

embryos in the experimental line were able to pip internally and externally, whereas the embryos in commercial lines were less able to pip internally and died before having free access to atmospheric oxygen and onset the pulmonary activation (DIL: BLE $3.6 \%$ vs. BLC 6.3\%, WLE $3.9 \%$ vs. WLC 9.3\%; DEL: BLE $1.4 \%$ vs. BLC $2.8 \%$, WLE $2.7 \%$ vs. WLC $7.7 \%$ ). During late embryonic development, anaerobic glycogenolysis is a major source of energy [47, 48]. Glycogen is synthesized during embryonic development through gluconeogenesis and is stored in the liver, heart, skeletal muscles, and in the musculus complexus (pipping muscle) and pectoralis in particular [19, 44, 45]. The metabolism and enzymatic activity of the musculus complexus increase continually until the day of hatching; a process that uses glycogen as its main source of energy. Accordingly, glycogen levels in the muscle cells are considerably reduced as the time of hatching approaches. Sharifi et al. [37] found a marked reduction in oxygen consumption and aerobic energy metabolism as compared with eggs bringing forth liveable chicks. Body, heart, liver, and pipping muscle growth rate, as well as glycogenolysis activity of the liver, heart and muscle in externally pipped embryos in lines selected for egg production, were lower than in the random control line $[49,50,51,52]$. The thyroid hormones play an essential role in the synthesis of glycogen and its accumulation in liver, heart and muscle tissues [53]. Therefore, the selection in pure lines for laying performance may influence embryonic metabolic pathways, and result in a retardation of the growth of organs and muscles involved in the hatching process, which in turn affects the pipping and embryonic survival ability.

Another factor which affects hatchability is embryonic malposition. Embryonic malposition has been proven to be related to internal pipping, external pipping and late embryonic mortality [26]. As shown in Figure 3.2, in failed hatched eggs, the proportion of malpositioned embryos was higher than the proportion of embryos poised in the normal hatching position in all lines. A higher incidence of malposition II, III and VI was observed in groups of embryos in an abnormal position, irrespective of investigated breeding lines. According to Ernst et al. [26], a large proportion of hatch failure is due to abnormal embryonic positions. Malposition VI has virtually no influence on hatching success and may reflect a normal variant position [12, 54]. Malposition III - head-under-left-wing - has been described to have a highly deleterious effect on hatchability $[28,45,55]$ and it is thought to be fatal in nearly all 


\section{Chapter 3}

cases [28, 54]. This is also the case, to a lesser extent, for malposition II [56]. The results of our study reveal that the incidence rate of late mortality is partly caused by abnormal embryonic positions II and III of embryos. The hatching process is characterized by initial pipping of the shell, followed by a rotational movement in a counter-clockwise direction of the embryo, chipping the shell up to $75 \%$ [10, 55, 57]. From position III, the embryo is able to pip both the air cell and the shell, but it is not easily able to break out of the shell due to a faulty head position [55]. Therefore, for BLC and BLE, malposition III is likely to be one of the main causes of late mortality. For WLC, the embryonic malposition seemed not to be the major cause for late embryonic mortality (LES: WLC $82.7 \%$ vs. BLC $84.1 \%$, WLE $85.5 \%$ vs. BLE 92.4\%; Incidence of malposition III: WLC $4.6 \%$ vs. BLC 32.9\%, WLE $12.0 \%$ vs. BLE 16.6\%). Wilson et al. [12] found no differences between different commercial genetic strains and lines according to the incidence of embryonic malpositions, which was partly in agreement with the current study. The estimated heritabilities for the incidence of malposition using a binomial traits (malpositioned compared to normally positioned embryo during late stage of development) were very low with exception of BLE (BLC: 0.02 \pm 0.01 ; WLC: $0.01 \pm 0.01$; BLE: $0.11 \pm 0.31$; WLE: $0.02 \pm 0.06$ ). Higher incidence of malpositions is often associated with high embryonic mortality. However, the impact of genetics on the incidence of faulty embryonic positions seems to be very low.

The hatchability of all egg sets and of fertilized eggs is presented in Table 3.1. The hatchability of fertilised eggs is more or less equal for WLC, WLE and BLC, ranging from $74.1 \%$ to $75.5 \%$. In contrast, the HFE rate for BLE was distinctly higher and reached $81.8 \%$. The hatchability of all eggs is determined by the following components: fertilization success, early, middle and late embryonic survival ability. For hatchability of fertilized eggs, the components of embryonic survival ability are determining factors. Fertilization and the phases of embryonic death occur at different stages of the reproduction process, and they are controlled by different environmental and genetic factors, which influence the functionality of pathways (metabolic pathway in particular) at late development, malformation and malposition. 

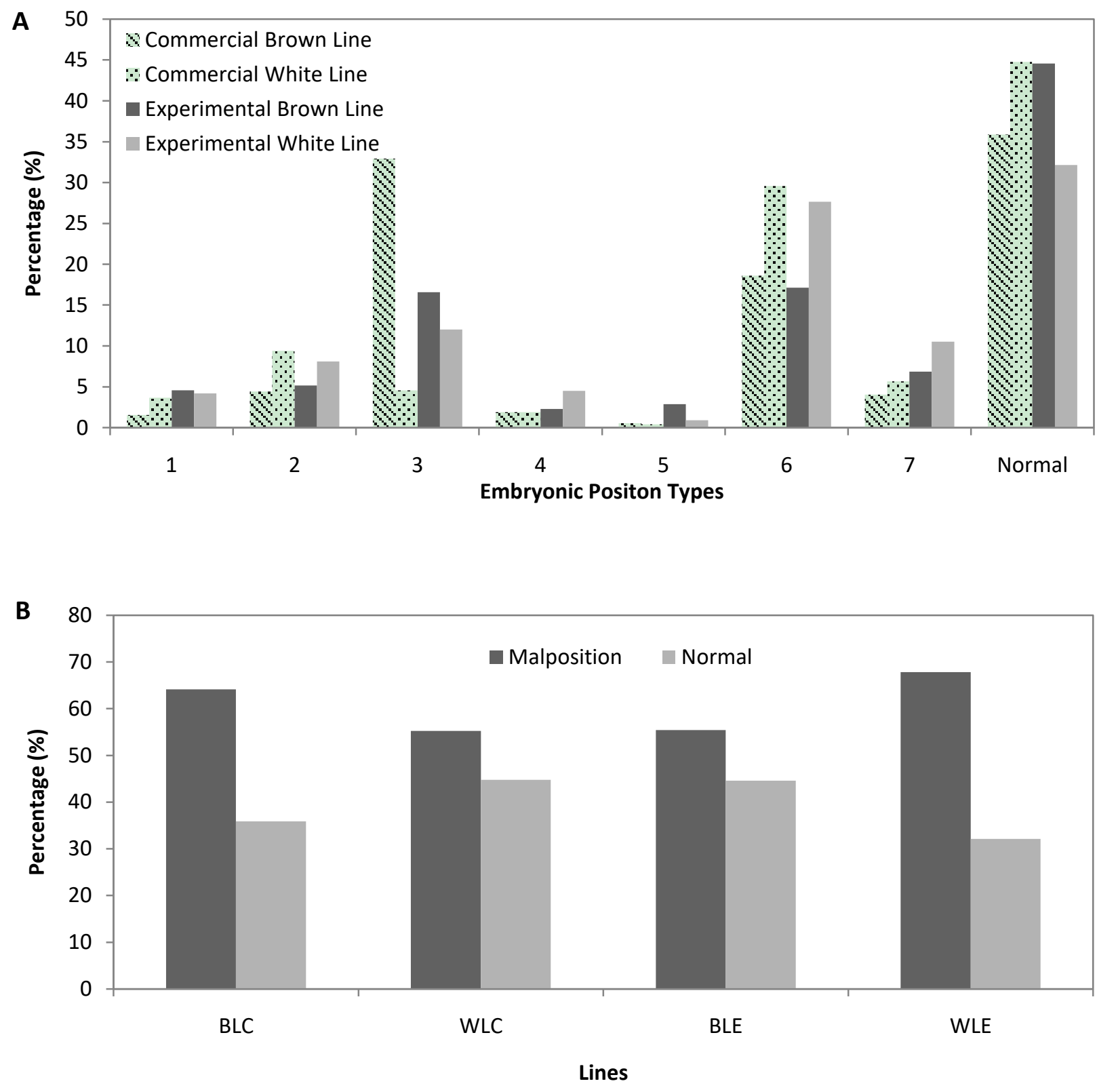

Figure 3.2: Percentage of different embryonic position types (A) and sum percentage of malpositions (B) for unhatched embryos late stage in the Commercial Brown Line, the Commercial White Line, the Experimental Brown Line and the Experimental White Line. Values are the percentage of specific malposition types among total unhatched embryos during late incubation stage. Position 1 is head between thighs. Position 2 is head in small end of the egg (opposite air cell). Position 3 is head under left wing. Position 4 is head in normal position but rotated with beak pointed away from air cell. Position 5 is feet over head. Position 6 is beak (or head) over right wing. Position 7 is embryo apart from the main axis of the egg.

The extent to which genetic factors impact upon hatching traits is presented in Table 3.2. The estimated genetic parameters reflect the genetic contribution of different physiological processes, acting at different stages of embryonic development, and may therefore be considered as distinct traits 


\section{Chapter 3}

$[2,29]$. In previous research studies, the estimation of genetic parameters was carried out using different statistical approaches. The estimated heritability on the underlying normally distributed scale obtained from sire component with Dempster and Lerner- transformation for FER, EES, MES, LES, HFE and HTE were $0.16,0.07,0.30,0.07,0.06$ and 0.08 . And using a logistic distribution obtained from a sire-dam model for FER, EES, MES, LES, HFE and HTE were 0.09, 0.09, 0.07, 0.05 and 0.05 [2].

Table 3.3:Estimation of genetic correlation for different reproductive traits hatchability of egg set (HTE), hatchability of fertile eggs (HFE), Fertility (FER), early stage survival ability (EES), middle stage survival ability (MES), late stage survival ability (LES), external pipping ability (EXP) in commercial lines

\begin{tabular}{|c|c|c|c|c|c|c|c|c|c|c|c|c|}
\hline & \multicolumn{6}{|c|}{ Commercial Brown Line } & \multicolumn{6}{|c|}{ Commercial White Line } \\
\hline & \multicolumn{6}{|c|}{ Reproductive traits } & \multicolumn{6}{|c|}{ Reproductive traits } \\
\hline & HFE & FER & EES & MES & LES & EXP & HFE & FER & EES & MES & LES & EXP \\
\hline HTE & $\begin{array}{c}0.97 \pm \\
0.01\end{array}$ & $\begin{array}{c}0.66 \pm \\
0.05\end{array}$ & $\begin{array}{c}0.72 \pm \\
0.03\end{array}$ & $\begin{array}{c}0.71 \pm \\
0.06\end{array}$ & $\begin{array}{c}0.79 \pm \\
0.01\end{array}$ & $\begin{array}{c}0.86 \pm \\
0.01\end{array}$ & $\begin{array}{c}0.99 \pm \\
0.01\end{array}$ & $\begin{array}{c}0.50 \pm \\
0.04\end{array}$ & $\begin{array}{c}0.73 \pm \\
0.03\end{array}$ & $\begin{array}{c}0.80 \pm \\
0.06\end{array}$ & $\begin{array}{c}0.94 \pm \\
0.01\end{array}$ & $\begin{array}{c}0.90 \pm \\
0.02\end{array}$ \\
\hline HFE & 0.01 & $\begin{array}{c}0.48 \pm \\
0.03\end{array}$ & $\begin{array}{c}0.64 \pm \\
0.04\end{array}$ & $\begin{array}{c}0.67 \pm \\
0.08\end{array}$ & $\begin{array}{c}0.93 \pm \\
0.01\end{array}$ & $\begin{array}{c}0.93 \pm \\
0.01\end{array}$ & 0.01 & $\begin{array}{c}0.41 \pm \\
0.04\end{array}$ & $\begin{array}{c}0.69 \pm \\
0.04\end{array}$ & $\begin{array}{c}0.81 \pm \\
0.06\end{array}$ & $\begin{array}{c}0.96 \pm \\
0.01\end{array}$ & $\begin{array}{c}0.93 \pm \\
0.01\end{array}$ \\
\hline FER & & - & $\begin{array}{c}0.83 \pm \\
0.03\end{array}$ & $\begin{array}{c}0.72 \pm \\
0.08\end{array}$ & $\begin{array}{c}0.21 \pm \\
0.05\end{array}$ & $\begin{array}{c}0.21 \pm \\
0.03\end{array}$ & & - & $\begin{array}{c}0.79 \pm \\
0.04\end{array}$ & $\begin{array}{c}0.41 \pm \\
0.09\end{array}$ & $\begin{array}{c}0.24 \pm \\
0.03\end{array}$ & $\begin{array}{c}0.27 \pm \\
0.05\end{array}$ \\
\hline EES & & & - & $\begin{array}{c}0.96 \pm \\
0.09\end{array}$ & $\begin{array}{c}0.32 \pm \\
0.05\end{array}$ & $\begin{array}{c}0.30 \pm \\
0.05\end{array}$ & & & - & $\begin{array}{c}0.60 \pm \\
0.09\end{array}$ & $\begin{array}{c}0.46 \pm \\
0.05\end{array}$ & $\begin{array}{c}0.46 \pm \\
0.04\end{array}$ \\
\hline MES & & & & - & $\begin{array}{c}0.34 \pm \\
0.08\end{array}$ & $\begin{array}{c}0.30 \pm \\
0.08\end{array}$ & & & & - & $\begin{array}{c}0.74 \pm \\
0.07\end{array}$ & $\begin{array}{c}0.87 \pm \\
0.10\end{array}$ \\
\hline LES & & & & & - & $\begin{array}{c}0.99 \pm \\
0.01\end{array}$ & & & & & - & $\begin{array}{c}0.95 \pm \\
0.01\end{array}$ \\
\hline
\end{tabular}

In the same study, the heritability estimates from dam component using a sire-dam model were distinctly higher for all reproductive traits in comparison to estimates obtained from sire component. The results of Beaumont et al. [2] were partly in agreement with Wolc et al. [32]. who focused on FER, HTE and HFE, and found dam heritability to be considerably higher for HFE only. In contrast, sire heritability was higher for HTE and FER. It has been argued that fertility is dependent on genetics and environmental factors originating from female and male. However, apart from environmental factors acting during egg storage and incubation, the embryonic survival ability (HFE) is significantly influenced by the embryo genotype and quality of egg [32]. In their study, fertility was recorded using candled non-broken eggs. And therefore, the estimated heritability for FER could be biased due to the 


\section{Chapter 3}

confounding effect of early embryonic mortality and fertility. In the case of HFE, the maternal genetic effect was distinctly larger (0.05 vs. 0.005). In our study, the heritabilities for FER were estimated based on broken eggs and ranged between 0.095 for BLC and 0.129 for WLC (see Table 3.2). As previously mentioned, using open eggs, higher sire heritability for fertility $(0.16)$ was noted by Lariviere et al. [29]. By using discharged candle eggs, similar or lower FER heritability values were obtained in other studies compared with results from this study $[2,9,32,58]$. In terms of the effect of selection for egg performance on fertility, there were essentially no differences between the different breeding lines, regardless of their selection history. These results demonstrate that selection for high performance and egg quality traits has no effect on genetic variation of fertility, although in other studies a negative genetic correlation was found between egg quality traits and fertility based on candling eggs $[3,5,32]$. In this study, the heritability estimates for embryonic survival ability at different stages of development ranged from 0.029 to 0.103 for EES, from 0.012 to 0.146 for MES and from 0.017 to 0.089 for LES. The estimated heritabilities for LES in commercial lines were higher than the estimated heritabilities for earlier stages of development, and they were the lowest for MES. In contrast, the highest heritabilities for MES were found in experimental lines. Previously reported heritability estimates for different stages of embryonic death (EES, MES and LES), when obtained from sire component, were generally low and similar to estimates provided in the present study $[2,59]$. However, as previously mentioned, Beaumont et al. [2] obtained distinctly higher heritability values for all stages of embryonic death using the dam component than using the sire component, and the values were $0.25,0.20$, and 0.18 for EES, MES and LES respectively. These results demonstrate that embryonic survival ability is mostly influenced by dam component through direct genetic effects passed to the embryo, and through the genetic component in order to provide suitable environmental conditions via the egg for growing embryo. Indeed, negative maternal genetic correlations have been reported between hatchability, and the egg weight and albumen weight quality traits, whereas positive correlations were found between hatchability and yolk weight, yolk proportion and albumen dry matter [60]. These results correspond to similar results from a selection experiment by Sewalem and Wilhelmson [61] who recorded a higher embryonic mortality in lines selected for egg weight compared to an unselected control line. The authors argued that the depressive effect of egg weight on 


\section{Chapter 3}

embryonic survival ability could be attributed to several factors including changes to the macrocomposition of eggs or reduced egg shell quality, as a result of an increase in egg size. Similar findings regarding the impact of egg weight on hatchability were reported by Cavero et al. [1]. Their research suggests that a larger egg size may mainly affect the late embryonic survival ability and also may result in a prolonged incubation time, which is in accordance with their practical experience. Wolc et al. [32] also discovered a negative genetic correlation between the hatchability of fertile eggs and egg weight, and a positive genetic correlation between hatchability and shell quality measured by specific gravity. In this article, the authors describe a negative impact of low specific gravity on early embryonic survival ability, as also demonstrated by McDaniel et al. [62]. In our study, the estimated heritabilities for LES in commercial lines were higher than the estimated heritabilities for earlier stages of development, and they were lowest for MES. In contrast, the highest heritabilities for MES were found in experimental lines. Thus, following analysis of the impact of selection for production efficiency on embryonic survival ability in this study, it became apparent that, between the experimental and commercial lines, there were no major differences in heritabilities for EES and LES, whereas distinct differences in estimated heritabilities for MES existed between the experimental and commercial lines. Therefore, it can be concluded that the selection for hatchability traits, applied in the commercial breeding program, resulted in a reduced genetic variation of middle embryonic survival ability. These results are in agreement with Lariviere et al. [29], who reported considerably higher heritability estimates for MES compared to estimates for other stage of embryonic mortality using traditional chicken breeding lines. This study shows the estimated heritabilities for HTE and HFE ranged from 0.044 to 0.079 for all four breeding lines, and do not differ substantially one from the other. These estimates are in agreement with previous studies, which show results ranging from 0.02 to $0.24[2,3,29,32,58,61,63]$. As compound traits, HTE and HFE are determined by contributing elements, embryonic survival ability at different stages of development and the fertilization process.Therefore, as expected, the estimated genetic correlation between HTE and HFE, FER, EES, MES, LES, EXP were positive for all breeding lines in this study (Table 3.3 and Table 3.4). Genetic correlations between HTE and HFE were positive and very high for all lines. Selection of HTE resulted in a correlated improvement in HFE, and selecting to improve the HFE caused an 


\section{Chapter 3}

improvement in HTE. The estimated genetic correlations between HFE and FER, and between HFE and EES for BLC, WLC and WLE lines, were moderate to high and ranged from 0.32 to 0.71 . For BLE these correlations were substantially higher (0.89 and 0.93). In contrast, the estimated genetic correlation between HFE and LES, and between HFE and EXP, was high for all lines, ranging from 0.68 upwards. A low to moderate genetic correlation was identified between FER and LES, as well as between FER and EXP (0.15 to 0.56). As expected, these results highlight that genetic potential for late embryonic survival ability and external pipping is an important factor for hatching success, whereas the genetic potential for fertilization process seems to be rather moderately or only loosely related to late survival ability and hatchability. These results correspond to a similar result reported by other researchers, who have found positive genetic correlations between HFE and FER, ranging from 0.20 to $0.71[2,29,32]$, as well as between HFE and LES (1.0) and FER and LES (0.29) [29]. Moreover, in our study, positive associations were also estimated between both FER and EES, and FER and MES. These results are consistent with results reported by Beaumont et al. [2], who reported a positive relationship between FER and EES, and between FER and MES driven from dam component. Lariviere et al. [29] however, found a negative genetic correlation between FER and EES, although the correlation between FER and MES was positive. According to Beaumont et al. [2], the genetic correlation between fertility and embryonic live ability at different stages of development is generally favourable, which is confirmed by the results of this study. Hence,selecting for increased fertility should result in decreased embryonic death. The estimates of genetic correlations between all stages of embryonic survival ability and external pipping were moderate to high and positive in this study, ranging from 0.30 to 0.99 . In literature, estimates of genetic correlations between the stages of embryonic survival ability are not consistent. As per the results of this study, and based on dam component, Beaumont et al. [2] estimated moderate to high genetic correlation between three stages of embryonic survival ability. These results are also in agreement with the study of Brah et al. [59], who estimated a strong genetic correlation between early mortality and late mortality, and a moderate relationship between both early mortality and pipping mortality, and between late and pipping mortality. However, Lariviere et al. [29] estimated a favourablepositive correlation between both EES and LES (0.34) and between MES and LES (0.11). However, a negative correlation was estimated 


\section{Chapter 3}

between EES and MES (-0.78). As highlighted by Lariviere et al. [29], discrepancies between different studies in estimates of genetic parameters could be attributed to breed differences or due to a different definition of mortality stages. Therefore, comparing genetic parameters with other studies is difficult due to different models being applied und differences in the genetics of the chicken population.

Table 3.4: Estimation of genetic correlation for different reproductive traits hatchability of egg set (HTE), hatchability of fertile eggs (HFE), Fertility (FER), early stage survival ability (EES), middle stage survival ability (MES), late stage survival ability (LES), external pipping ability (EXP) in experimental lines

\begin{tabular}{|c|c|c|c|c|c|c|c|c|c|c|c|c|}
\hline & \multicolumn{6}{|c|}{ Experimental Brown Line } & \multicolumn{6}{|c|}{ Experimental White Line } \\
\hline & \multicolumn{6}{|c|}{ Reproductive traits } & \multicolumn{6}{|c|}{ Reproductive traits } \\
\hline & HFE & FER & EES & MES & LES & EXP & HFE & FER & EES & MES & LES & EXP \\
\hline HTE & $\begin{array}{c}0.99 \pm \\
0.01\end{array}$ & $\begin{array}{c}0.93 \pm \\
0.01\end{array}$ & $\begin{array}{c}0.94 \pm \\
0.02\end{array}$ & $\begin{array}{c}0.72 \pm \\
0.12\end{array}$ & $\begin{array}{c}0.67 \pm \\
0.12\end{array}$ & $\begin{array}{c}0.77 \pm \\
0.13\end{array}$ & $\begin{array}{c}0.98 \pm \\
0.01\end{array}$ & $\begin{array}{c}0.47 \pm \\
0.14\end{array}$ & $\begin{array}{c}0.72 \pm \\
0.07\end{array}$ & $\begin{array}{c}0.87 \pm \\
0.10\end{array}$ & $\begin{array}{c}0.93 \pm \\
0.02\end{array}$ & $\begin{array}{l}0.95 \pm \\
0.023\end{array}$ \\
\hline HFE & - & $\begin{array}{c}0.89 \pm \\
0.03\end{array}$ & $\begin{array}{c}0.93 \pm \\
0.03\end{array}$ & $\begin{array}{c}0.73 \pm \\
0.13\end{array}$ & $\begin{array}{c}0.68 \pm \\
0.11\end{array}$ & $\begin{array}{c}0.75 \pm \\
0.12\end{array}$ & & $\begin{array}{c}0.32 \pm \\
0.16\end{array}$ & $\begin{array}{c}0.71 \pm \\
0.07\end{array}$ & $\begin{array}{c}0.88 \pm \\
0.10\end{array}$ & $\begin{array}{c}0.96 \pm \\
0.02\end{array}$ & $\begin{array}{c}0.98 \pm \\
0.02\end{array}$ \\
\hline FER & & - & $\begin{array}{c}0.93 \pm \\
0.04\end{array}$ & $\begin{array}{c}0.90 \pm \\
0.10\end{array}$ & $\begin{array}{c}0.47 \pm \\
0.19\end{array}$ & $\begin{array}{c}0.56 \pm \\
0.22\end{array}$ & & . & $\begin{array}{l}0.31 \pm \\
0.016\end{array}$ & $\begin{array}{c}0.36 \pm \\
0.22\end{array}$ & $\begin{array}{c}0.21 \pm \\
0.18\end{array}$ & $\begin{array}{c}0.29 \pm \\
0.18\end{array}$ \\
\hline EES & & & - & $\begin{array}{c}0.73 \pm \\
0.15\end{array}$ & $\begin{array}{c}0.38 \pm \\
0.18\end{array}$ & $\begin{array}{c}0.52 \pm \\
0.20\end{array}$ & & & - & $\begin{array}{c}0.33 \pm \\
0.20\end{array}$ & $\begin{array}{c}0.52 \pm \\
0.12\end{array}$ & $\begin{array}{c}0.58 \pm \\
0.12\end{array}$ \\
\hline MES & & & & - & $\begin{array}{c}0.30 \pm \\
0.24\end{array}$ & $\begin{array}{c}0.35 \pm \\
0.26\end{array}$ & & & & - & $\begin{array}{c}0.93 \pm \\
0.12\end{array}$ & $\begin{array}{c}0.93 \pm \\
0.15\end{array}$ \\
\hline LES & & & & & - & $\begin{array}{c}0.98 \pm \\
0.03\end{array}$ & & & & & - & $\begin{array}{c}0.99 \pm \\
0.01\end{array}$ \\
\hline
\end{tabular}

Means and the range of estimated breeding values are presented in Figure 3.3. The estimated breeding values for fertility vary between $84.6 \%$ and $98.0 \%$ in all lines, and the values for hatchability of fertile eggs vary between $48.1 \%$ and $98.0 \%$. The most significant differences in magnitude of estimated breeding values for FER, HTE, HFE and LES were found in WLC $(23 \%, 39 \%, 34 \%$ and $36 \%)$ and the less significant in BLE $(8 \%, 23 \%, 16 \%$ and $1 \%)$. The low estimated heritability of EES, MES and LES may result in a low selection response during the breeding procedure. However, considering the high reproductive performance and low generation interval in chickens, and the wide range in magnitude of estimated breeding hatchability values (Figure 3.3), it appears to be feasible to successfully select animals for increased embryonic viability. 
Chapter 3
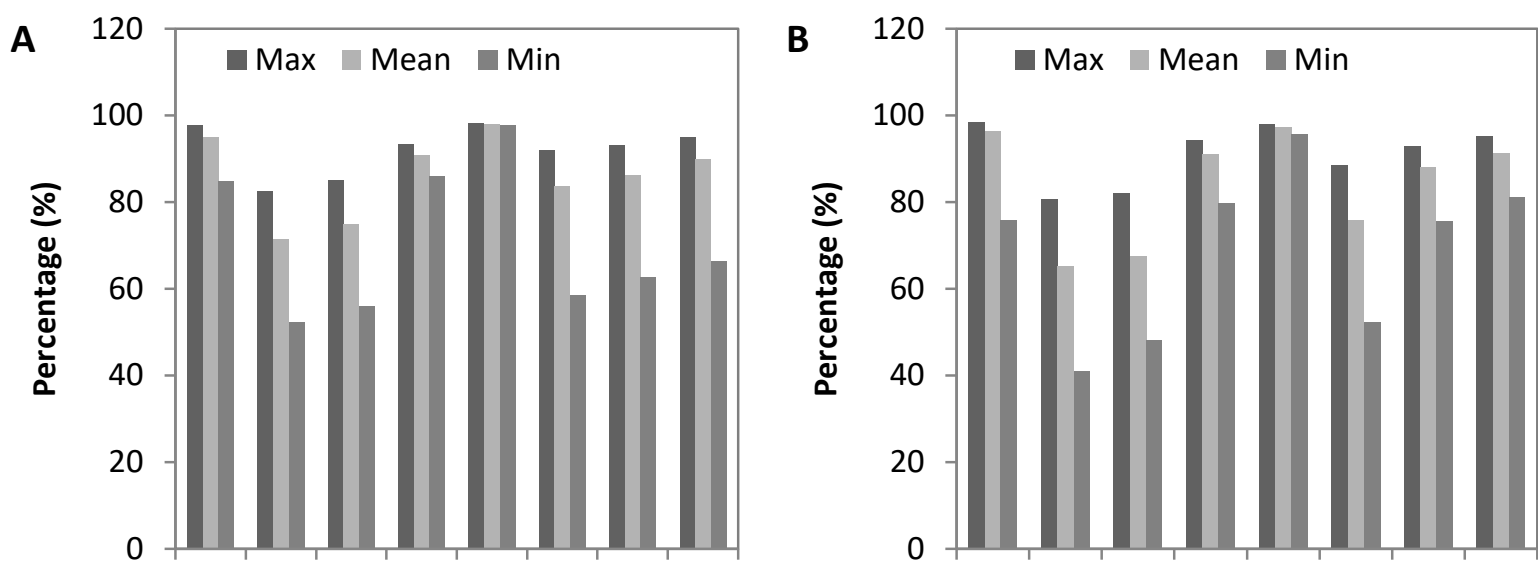

FER HTE HFR EES MES LES EXP INT

FER HTE HFR EES MES LES EXP INT

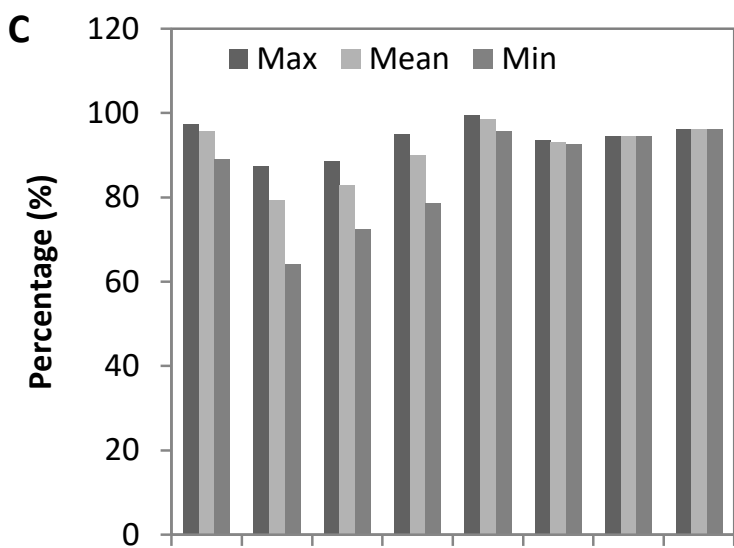

FER HTE HFR EES MES LES EXP INT

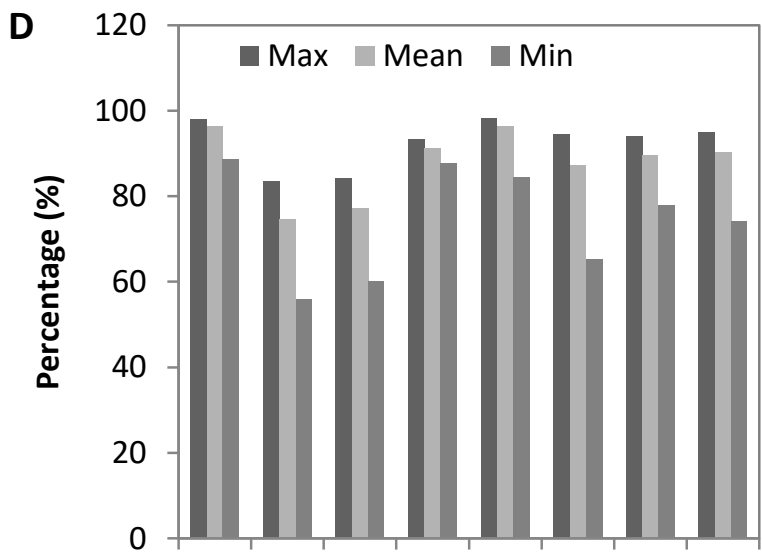

FER HTE HFR EES MES LES EXP INT

Figure3.3:Estimated breeding values of hatchability traits in the Commercial Brown Line (A), the Commercial White Line (B), the Experimental Brown Line (C) and the Experimental White Line (D). Values are the maxima, means and minima of estimated breeding values from generalized linear mixed models.

As previously mentioned, the curse of embryonic death could be distinguished in three different stages due to varying anatomical changes and physiological needs, which are specific to these phases of development. Apart from environmental influences, genetic factors involved in the embryonic developmental process, which affect embryonic viability at different stages, may differ in heritability resulting in more efficient selection for embryonic survival ability. Selection for high performance may affect associated fitness traits, such as embryonic survival ability, by modifying maternal traits and deposition of micro and macronutrients to eggs [61]. Therefore, breeding strategies applied to different populations may lead to differences in genetic predisposition for embryonic death at certain developmental stages and fertility disorder, which in turn require selection strategies based on these components. The estimates of genetic parameters in this study, carried out on different populations 


\section{Chapter 3}

differing in breeding history, together with previous results from literature, indicate that a complex trait like HTE can be used as selection criteria for improving reproductive performance. In cases where a negative correlation between these different components exists, a selection index approach should be applied.

\subsection{Conclusion}

Open egg inspection as a method for determining the reason for embryonic death is a more credible technique than the classical candling method. In addition, this technology makes it possible to determine embryonic malpositions and malformation. Genetic parameter estimation results suggest that heritabilities for FER, HTE, HFE, EES, MES, LES, EXP and INP are generally low. These results indicate that the potential selection response will be limited. However, considering the high reproductive performance and low generation interval in chickens, as well as the wide range in magnitude of estimated breeding values for hatchability, successfully selecting animals for increased embryonic survival appears to be a feasible endeavor.

\subsection{Summary}

Past research determined the time of embryonic death and mortality based on candling of hatching eggs rather than opening the eggs to determine the exact time of embryonic death to ensure thorough biological assessment. This technique could generate a biased result when estimating genetic parameters due to imprecise recording of embryonic mortality and fertility. This study aims to apply a new methodology for determining mortality and estimating genetic parameters in relation to fertility (FER), hatchability of total eggs set (HTE) and fertilized eggs (HFE), susceptibilities to early (EES), middle (MES), late (LES) embryonic mortality, external pipping ability (EXP) and internal pipping ability (INP) using different populations of laying hens with varying breeding histories. Data sets consist of 5,983 hatching egg records obtained from 100 unselected experimental brown laying hens (BLE) and 99 white laying hens (WLE) from Friedrich Loeffler Institute (FLI), and 80,916 egg records selected from 2909 brown laying hens (BLC) and 3319 white laying hens (WLC) from Lohmann company. To determine the time of embryonic mortality, eggs not exhibiting a viable 


\section{Chapter 3}

embryo at candling on days 7 and 18 of incubation, and eggs that did not hatch, were opened and examined macroscopically to assess their true fertility and estimate the time of embryonic death. Heritabilities were estimated using a generalized linear animal model and results suggest that heritability for FER, HTE, HFE, EES, MES, LES, EXP and INP exhibit ranges of 0.10-0.13, 0.04-0.08, $0.05-0.06,0.03-0.10,0.01-0.18,0.02-0.09,0.001-0-083$ and $0.001-0.11$, respectively. The estimated genetic correlations between different reproductive traits for all breeding lines were positive ranging from 0.94 to 0.15 . The gaps between minimum and maximum breeding values of HTE and HFE were large and on average approximately $30 \%$. These low heritability traits led to the conclusion that the potential selection response is expected to be limited. Taking into consideration the very high reproductive ability and very short generation interval in chicken, it is feasible to select animals for improved hatchability.

\subsection{References}

1. Cavero D, Schmutz M. Proceedings of the European Poultry Symposium on Quality of Poultry Meat and Quality of Eggs and Egg Products. Turku Finland 2009.

2. Beaumont C, Millet N, Le Bihan-Duval E, Kipi A, Dupuy V. Genetic parameters of survival to the different stages of embryonic death in laying hens. Poultry Sci 1997;76:1193-1196.

3. Sewalem A, Johansson K, Carlgren AB, Wilhelmson M, Lillpers K. Are reproductive traits impaired by selection for egg production in hens? J Anim Breed Genet 1998;115:281-297.

4. Dobek A, Szydlowski M, Szwaczkowski T, Skotarczak E, Molinski K. Bayesian estimates of genetic variance of fertility and hatchability under a threshold animal model. J Anim Feed Sci $2003 ; 12: 307-314$

5. Rozempolska-Ruciska I, Zieba G, Łukaszewicz M. Hatchability traits as selection criteria in breeding of laying hens. Arch Geflugelkd 2008;73:263-267.

6. Wondmeneh E, Dawud I, Adey M. Comparative evaluation of fertility and hatchability of Horro, Fayoumi, Lohmann Silver and Potchefstroom Koekoek breeds of chicken. Asian J Poultry Sci 2011;5:124-129. 
7. FAO. 2015. Database. http://faostat.fao.org/site/569/DesktopDefault.aspx?PageID=569\#ancor Accessed April 16, 2016.

8. Schaal T, Cherian G. A survey of the hatchability of broiler and turkey eggs in the United States from 1985 through 2005. Poultry Sci 2007;86:598-600.

9. Bennewitz J, Morgades O, Preisinger R, Thaller G, Kalm E. Variance component and breeding value estimation for reproductive traits in laying hens using a Bayesian threshold model. Poult. Sci 2007;86:823-828.

10. Moran ET. Nutrition of the developing embryo and hatchling. Poultry Sci 2007;86:1043-9.

11. Leeson S, Reinhart BS, Summers JD. Response of white leghorn and Rhode Island red breeder hens to dietary deficiencies of synthetic vitamins .2. Embryo mortality and abnormalities. Can J Anim Sci 1979;59:569-575.

12. Wilson HR. Hatchability Problem Analysis. Univ of Florida IFAS Extension 2004.

13. Liptoi K, Hidas A. Investigations of possible genetic background of early embryonic mortality in poultry. World Poultry Sci J 2006;62:326-337.

14. Harry EG. The effect of embryonic and chick mortality of yolk contamination with bacteria from the hen. Vet Rec 1957;69:110-120.

15. Fasenko GM, Robinson FE, Armstrong JG, Church JS, Hardin RT, Petitte JN. Variability in preincubation embryo development in domestic fowl. 1. Effects of nest holding time and method of egg storage. Poultry Sci 1991;70:1876-1881.

16. Lerner SP, French N, Mclntyre D, Baxter-Jones C. Age-related changes in egg production, fertility, embryonic mortality, and hatchability in commercial turkey flocks. Poultry Sci 1993;72:10251039.

17. Kirk S, Emmans GC, Mcdonald R, Arnot D. Factors affecting the hatchability of eggs from broiler breeders. Brit Poultry Sci 1980;21:37-53.

18. Tullett SG. Science and the art of incubation. Poultry Sci 1990;69:1-15.

19. Christensen VL. Factors associated with early embryonic mortality. World Poultry Sci J 2001;57:359-372. 


\section{Chapter 3}

20. Cahaner A, Abplanalp H, Shultz FT. Effects of inbreeding on production traits in turkeys. Poultry Sci 1980;59:1353-1362.

21. Ibe SN, Rutledge JJ, Mcgibbon WH. Inbreeding effects on traits with and without selection for part record rate of lay in chickens. Poultry Sci 1983;62:1543-1547.

22. Flock DK, Ameli H, Glodek P. Inbreeding and heterosis effects on quantitative traits in a white leghorn population under long-term reciprocal recurrent selection. Brit Poultry Sci 1991;32:451462.

23. Biémont C. A brief history of the status of transposable elements: from junk DNA to major players in evolution. Genetics 2010;186:1085-1093.

24. Kuurman, WW, Bailey BA, Koops WJ, Grossman M. A model for failure of a chicken embryo to survive incubation. Poultry Sci 2003;82:214-222.

25. Hamburger V, Hamilton HL. A series of normal stages in the development of the chick embryo. $\mathrm{J}$ Morphol 1951;88:49-92.

26. Ernst RA, Bradley FA, Delany ME, Abbott UK, Craig RM. Egg candling and breakout analysis. ANR Publication 8134, Univ of California 2004.

27. Romanoff AL. Pathogenesis of the Avian Egg. Wiley-Inter-science, New York, NY 1972.

28. Landauer W. The Hatchability of Chicken Eggs as Influenced by Environment and Heredity. Agricultural Experiment, Storrs Station Monograph 1967;1:68-137.

29. Larivière JM, Michaux C, Leroy $\mathrm{P}$. Genetic parameters of embryonic viability traits in a traditional chicken breed. Int J Poultry Sci 2009;8:1183-1188.

30. SAS Institute. SAS Users's Guide: Statistics. Version 9.3 ed. SAS Institute Inc., Cary NC 2012.

31. Gilmour AR, Gogel BJ, Culis BR, Thompson R. Asreml User Guide. Release 2.0. Vsn International Ltd. Hemel Hempstead UK 2006.

32. Wolc A, White IM, Hill WG, Olori VE. Inheritance of hatchability in broiler chickens and its relationship to egg quality traits. Poultry Sci 2010;89:2334-40.

33. Alsobayel AA, Albadry MA. Effect of age and sex ratio on fertility and hatchability of Baladi and Leghorn laying hens. J Anim Plant Sci 2012;22:15-19. 


\section{Chapter 3}

34. Siegel PB, Dunnington EA. Reproductive complications associated with selection for broiler growth. Pages 59-72 in: Poultry Genetics and Breeding. W. G. Hill, J. H. Manson, and D. Hewitt, ed. Longman Group Harlow UK 1985.

35. Rozempolska-Rucińska I, Zięba G, Twardowska M, Łukaszewicz M, Witkowski A. Relationships between hatchability and routine selection criterion in laying hens. Proc 19th Int Poultry Sci PB WPSA Olsztyn 2007.

36. Kuurman WW, Bailey BA, Koops WJ, Grossman M. Influence of storage days on the distribution for time of embryonic mortality during incubation. Poultry Sci 2002;81:1-8.

37. Sharifi AR, Horst $\mathrm{P}$, Simianer $\mathrm{H}$. The effect of naked neck gene and ambient temperature and their interaction on reproductive traits of heavy broiler dams. Poultry Sci 2010;89:1360-1137.

38. Hutt FB, Pilkey AM. Studies in embryonic mortality in the fowl, V. Relationships between positions of the egg and frequency of malpositions. Poultry Sci 1934;13:3-13.

39. Zakaria AH, Plumstead PW, Romero-Sanchez H, Leksrisompong N, Brake J. The effects of oviposition time on egg weight loss during storage and incubation, fertility, and hatchability of broiler hatching eggs. Poultry Sci 2009;88:2712-2717.

40. Samiullah, S, Roberts J, Chousalkar K. Oviposition time, flock age, and egg position in clutch in relation to brown eggshell color in laying hens. Poultry Sci 2016;95:2052-2057.

41. Coleman JW, Siegel PB. Selection for body weight at eight weeks of age: Embryonic state at oviposition and its relationship to hatchability. Poultry Sci 1966;45:1008-1011.

42. Jaap RG, Fechheimer NS. Normal and abnormal avian chromosomes. Proc 15th World Poult Cong New Orleans 1974.

43. Gwaza DS, Dim NI, Momoh OM. Evaluation of Sire and Dam for Genetic Improvement of Fertility, Embryonic Mortality and Hatchability in Nigerian Local Chickens Populations. Adv Genet Eng 2016;5: 2169-0111.

44. De Oliveira JE, Uni Z, Ferket PR. Important metabolic pathways in poultry embryos prior to hatch. World Poultry Sci J 2008;64:488-99. 
45. Pulikanti R, Peebles ED, Keirs RW, Bennett LW, Keralapurath MM, and Gerard PD. Pipping muscle and liver metabolic profile changes and relationships in broiler embryos on days 15 and 19 of incubation. Poultry Sci 2010;89:860-865.

46. Rahn, H. 1981. Gas exchange of avian eggs with special reference to turkey eggs. Poult. Sci. 60:1971-1980.

47. Freeman BM. The importance of glycogen at the termination of the embryonic existence of Gallus domesticus. Comp Biochem Physiol 1965;28:1169-1171.

48. Christensen VL, Donaldson WE. Effect of oxygen and maternal dietary iodine on embryonic carbohydrate metabolism and hatchability of turkey eggs. Poultry Sci 1992;71:747-53.

49. Christensen VL, Donaldson WE, Nestor KE. Effect of supplemental oxygen on blood plasma organic acids within embryos from selected lines of turkeys. Poultry Sci 1999;78:1601-5.

50. Christensen VL, Donaldson WE, Nestor KE, McMurtry JP. Effect of genetics and maternal dietary iodide supplementation on turkey embryonic growth. Poultry Sci 1999;78:883-889.

51. Christensen VL, Donaldson WE, Nestor KE. Embryonic viability and metabolism in turkey lines selected for egg production or growth. Poultry Sci 1993;72:829-838.

52. Christensen VL, Nestor KE. Changes in functional qualities of turkey eggshells in strains selected for increased egg production or growth. Poultry Sci 1994;73:1458-1464.

53. Christensen VL, Donaldson WE, McMurtry JP. Physiological differences in late embryos from turkey breeders at different ages. Poultry Sci 1996;75:172-178.

54. Wilson HR, Neuman SL, Eldred AR, Mather FB. Embryonic Malpositions in Broiler Chickens and Bobwhite Quail. 2003.

55. Byerly TC, Olsen MW. Causes of the embryonic malposition head-under-left-wing. Poultry Sci $1934 ; 13: 278-282$.

56. Talmagde WT. The effect of incubating eggs narrow end up on malposition II and hatchability. Poultry Sci 1977;56: 1046-1048.

57. Decuypere E, Willemsen H. Physiological control mechanisms during late embryogenesis and during pipping and hatching. Post Graduation Course in Incubation Biology and Management. Univ of Wageningen Holland 2006. 


\section{Chapter 3}

58. Sapp RL, Rekaya R, Misztal I, Wing T. Male and female fertility and hatchability in chickens: A longitudinal mixed model approach. Poultry Sci 2004;83:1253-1259.

59. Brah GS, Sandhu JS, Chaudhary ML. Heritability estimates of components of incubation mortality in White Leghorns. Brit Poultry Sci 1991;32:871.

60. Hartmann C, Strandberg E, Rydhmer L, Johansson K. Genetic Relations between Reproduction, Chick Weight and Maternal Egg Composition in a White Leghorn Line. Acta Agric Scand A 2002;52:91-101.

61. Sewalem A, Wilhelmson M. Genetic study of embryonic mortality in white leghorn lines selected for egg production traits. Brit Poultry Sci 1999;40:467-471.

62. McDaniel GR, Brake J, Eckman MK. Factors affecting broiler breeder performance.The interrelationship of some reproductive traits. Poultry Sci 1981;60:1792-1797.

63. Beaumont C. Comparison of Henderson's method I and restricted maximum likelihood estimation of genetic parameters of reproductive traits. Poultry Sci 1991;70:1462-1468. 


\title{
CHAPTER 4 METABOLITE PROFILE OF CHICKEN
}

\author{
EGG YOLK AND ITS ASSOCIATION WITH
}

\section{HATCHABILITY}


Chapter 4

\section{Metabolite Profile of Chicken Egg Yolk and Its Association with}

\section{Hatchability}

\subsection{Introduction}

Hatchability is an important trait for the chicken industry because of its high impact on chicken output. Based on egg fertility, hatchability can be divided into two groups: 1 . hatchability relative to the total number in the egg set; and 2. hatchability of fertilized eggs [1]. Previous research has surmised that fertility mainly relies on the parents' genetics and environmental factors. In contrast, hatchability or embryonic survival ability in fertile eggs is highly influenced by the offspring genotype and the quality of the egg environment, such as egg nutritional content [1, 2]. Different embryonic development stages have distinct causes of embryonic mortality [3]. The frequency of embryonic death is not equally distributed over the course of incubation and can be distinguished in three distinct stages: early embryonic mortality occurs during the first week of incubation, middle embryonic mortality occurs after the first week of incubation and before transfer of the eggs into hatcher, and late embryonic mortality. These stages are characterized by physiological factors and changes during embryonic development. Moran in 2007 [4] indicates that in the early stage (the first week), the embryo is developing a vascular system and attempting to complete chorioallantois. Thus, $\mathrm{O} 2$ access is limited, and energy supplementation relies on the glycolysis of accessible glucose. After the vascular system is completed, the embryo could utilize other nutrients in egg. Fatty acid from the yolk is the most important energy source for completion of the embryo in its final form [4]. At 17th day of incubation, the maximum of chorioallantois respiration capacity is reached, defined as the plateau stage of oxygen consumption. During the plateau stage after day 15 of incubation, the energetic metabolism switches from using yolk fat to carbohydrate. In the last stage before emergence or pipping out, the embryo must absorb a large amount of protein and fatty acids to develop muscle in order to produce energy and store glycogen $[4,5]$. Particularly during last week of incubation approximately $80 \%$ of total lipid content of the yolk will be mobilized and absorbed into the embryonic tissues. Moreover, during the embryonic development $90 \%$ of the total energy 


\section{Chapter 4}

requirements of the embryo are provided by $\beta$-oxidation of fatty acids derived from the yolk [6]. Therefore the metabolic pathways that are related to lipids are most active during poultry embryonic development $[4,6]$.

Egg yolk composes approximately $36 \%$ of the total fresh chicken egg and is the portion with the greatest nutritional value. The main components of the yolk are lipids (approximately $65 \%$ of the dry matter). Protein composes only approximately $33 \%$ of the yolk [7]. In terms of the fatty acid composition of the lipids, approximately $30-35 \%$ consists of saturated fatty acids (SFAs), 40-45\% consists of monounsaturated fatty acids (MUFAs), and 20-25\% consists of polyunsaturated fatty acids (PUFAs) [7]. Among these components, the main fatty acids are oleic acid (C18:1, 40-45\%), palmitic acid (C16:0, 20-25\%), and linoleic acid (C18:2, 15-20\%) [8], all of which may have a relationship with egg hatchability [8]. The embryonic survival ability is highly associated with a 2- to 3-fold decrease in desaturated ratios (cis-9 C16:1/C16:0 and cis-9 C18:1/C18:0) in the total egg yolk lipids [9, $10,11]$. Increasing the dietary linoleic acid has a compensatory effect on the hatchability decrease due to the reduced levels of palmitoleic and oleic acids [9]. The dietary linoleic acid could act as a precursor of the C20-22 PUFAs, particularly for the generation of C20:4 n-6 arachidonic acid, which has a similar function as C22:6 n-3 docosahexaenoic acid in the stimulation of embryonic neural system development $[10,11]$. Among avian species, chicken is a special species that has a precocial hatching mode. This advanced type of development allows newly hatched chickens to quickly become active and mobile, grow plumage, open their eyes and regulate their body temperature [12]. Therefore a high presentation of docosahexaenoic acid is important in the physiological functioning of neural cell during the embryonic development [12]. Apart from fatty acids, Kadam et al. [13] observed that chick embryo weight tended to be lower when eggs in ovo were injected with excessive threonine. They concluded that the higher dose could have caused an amino acid imbalance [13]. Vitamins, such as biotin and folic acid, have antitoxic effect, cell division stimulation, immune system enhancement during embryonic development in chickens in both early and late stages [14, 15]. A certain level of cholesterol in egg yolk is required for embryonic development and attempts to lower this level would 


\section{Chapter 4}

result in reduced hatchability [16]. The main goals of this study are to determine nutrition profiles in the egg yolk and to assess possible associations with embryonic mortality.

\subsection{Material and Methods}

\subsubsection{Study Population and Design}

Previous research has examined the breeding values of the following hatchability traits: hatchability of all egg sets (HTE), hatchability of fertilized egg sets (HFE), early stage of embryonic survival ability (EES, from the 1st to 7th day of incubation), middle stage of embryonic survival ability (MES, from the 8 th to 17 th day of incubation), late stage of embryonic survival ability (LES, from the 18th day to hatch) and external pipping ability (EXP); these traits have been estimated for both commercial (Lohmann Tierzucht $\mathrm{GmbH}$ ) and experimental populations (Friedrich-Loeffler-Institut, Germany), totaling 3319 commercial white laying hens (WLC), 2909 commercial brown laying hens (BLC), 99 experimental white laying hens (WLE) and 100 experimental brown laying hens (BLE). Then, based on the HTE breeding values, these animals were divided into high and low breeding value groups (HBV and LBV). Approximately an equal number of chickens from the two groups were chosen for metabolite profiling. In the metabolite profile experiment, 1069 yolk samples of 382 WLC and 1014 yolk samples of 250 BLC were collected to generate metabolite profiles. In the case of experimental lines, 257 yolk samples of 90 WLE and 238 yolk samples of 81 BLE hens were used. The two groups were fed with different compounded feeding components based on a mixture of maize, wheat, soybean and rapeseed supplemented with vegetable oils and different food additives derived from Lohmann

Tierzucht GmbH (Cuxhaven) and AGRAVIS Mischfutter Ostwestfalen-Lippe GmbH (Minden) (Figure 4.1). 


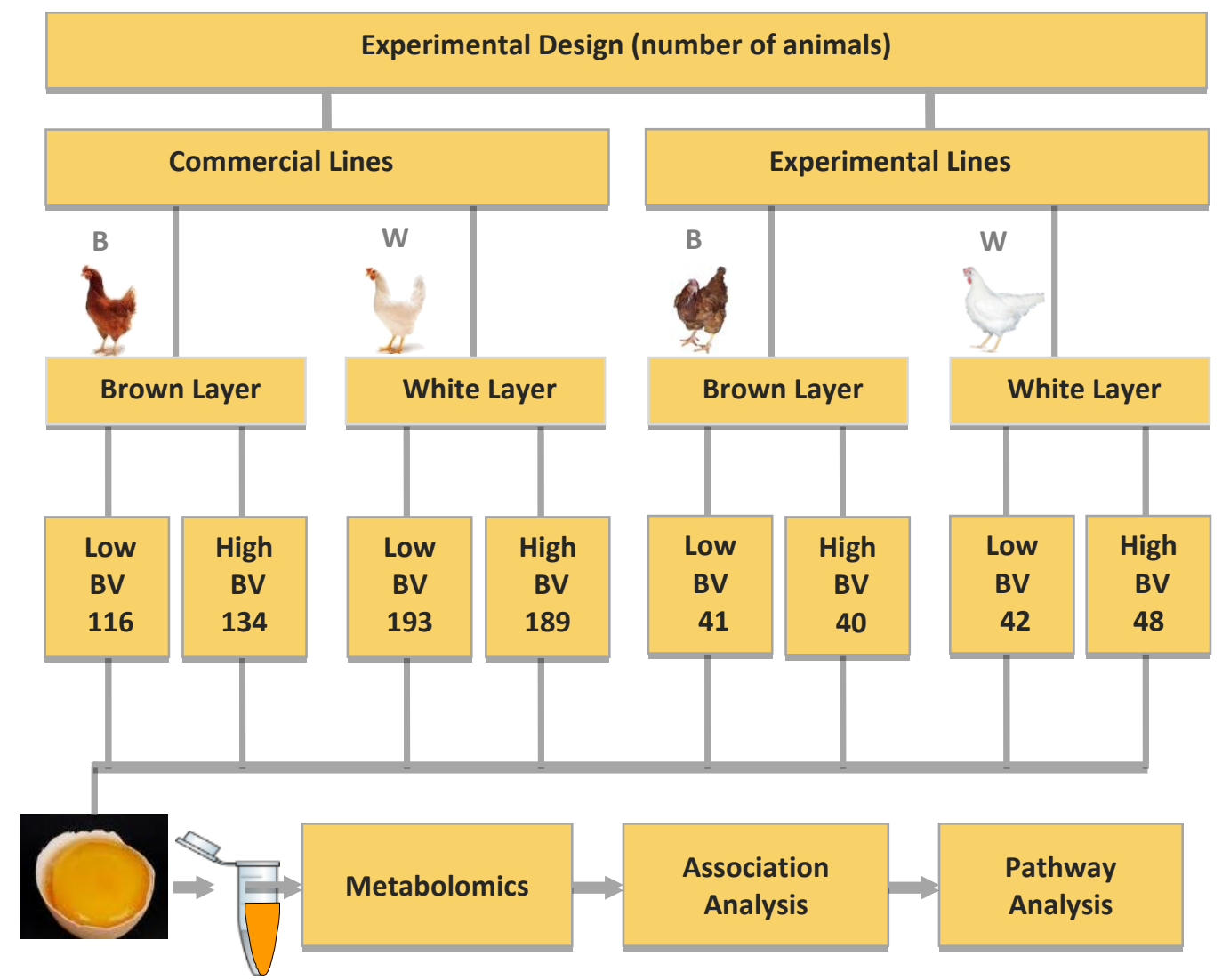

Figure 4.1: Experimental design of chicken egg yolk metabolite profile experiment.

\subsubsection{The Determination of Metabolite Profile in Egg Yolk and Data Processing}

For metabolite profiling, 50-mg fresh egg yolk samples on five consecutive days (from 1 to 5 egg yolks from the same hen) were simultaneously collected, snap-frozen in liquid nitrogen and stored under argon at $-70^{\circ} \mathrm{C}$. Commercial line yolk samples were taken from unfertilized eggs of 53-weekold. Experimental line yolk samples used for metabolite analysis were taken from unfertilized eggs of 52-week-old hens. For chemical analysis, two random eggs per hen were combined after metabolite extraction. The order of the sample extraction and chromatographic analysis was performed strictly according to the randomization scheme based on the alpha block design. Total metabolite extraction was performed with the modified method of Fiehn and Kind [17] in three consecutive steps for every individual yolk sample. The three extracts from each sample were combined, and sample pooling was performed after the extraction. Three aliquots of 5,160 and $500 \mu \mathrm{L}$ were made in $1.1 \mathrm{~mL}$ conical glass 


\section{Chapter 4}

vials and dried overnight in a rotary vacuum concentrator (Saur Laborbedarf, Reutlingen, Germany) at 2 mbar pressure and room temperature (RT). The chemical derivatization of the $500-\mu \mathrm{L}$ aliquots and the gas chromatography/time-of-flight mass spectrometry (GC-TOFMS) analysis of silylated samples were performed according to Lisec et al [18]. Next, 5- and 160- $\mu \mathrm{L}$ samples were dissolved in $100 \mu \mathrm{L}$ of chloroform containing $13 \mathrm{n}$-alkanes $(1 \mu \mathrm{g}$ each) ranging from $\mathrm{C} 11$ to $\mathrm{C} 35$ (only odd carbon numbers) as retention index standards. For chemical derivatization, $50 \mu \mathrm{L}$ of trimethylsulfonium hydroxide (TMSH) was added to these samples. Data preprocessing and peak quantification were performed using the instrument software ChromaTOF (4.24) in the methylated samples and using XCMS (1.3) and CAMERA (1.11.3) software in the silylated samples. Signal characterization was performed using ChromaTOF (4.24) software and NIST08 and FAMEs mass spectral databases and an in-house compound library. Because the signal intensity correlated with the amount of the special metabolite, we determined the peak area of the selected ions for each of the metabolites in the samples. Then, we applied this peak area data (no unit) as semi-quantitative data for further analysis.

\subsubsection{Data Normalization and Association Analysis}

The first part of the association analysis was between the metabolites and the HTE. Then, the metabolite data were used in the next step of the analysis with other hatching traits: HFE, EES, MES, LES and EXP.

Before the association analysis, the metabolite data were adjusted according to the p-rep design used for metabolite profiling [19], with blocks corresponding to days. In the adjusted model, the peak area data of the metabolites were log-transformed. In this model, identification of animal was taken as fixed effect. The effects that were related to the environment of the hens, i.e., the house, lane, side and floor, were taken as random. The effects that were related to the laboratory, i.e., the block (day) and position of the analysis of the sample, were considered to be random and covariate, respectively. The statistical model for the commercial line is presented below:

$$
\log \left(y_{i j k l m o}\right)=\mu+A_{i}+B_{j}+H_{k}+b(B P)+R_{1}(H)+S_{m}(H)+E_{n}(H)+e_{i j k l m n o}
$$




\section{Chapter 4}

Where $y_{i j k l m o}$ is the content of a certain metabolites; $\mu$ is the overall means; $A_{i}$ is fixed effect of animal identification; $B_{j}$ is the random effect of block; $H_{k}$ is the random effect of house; $b$ is the linear regression coefficient of time on block position; $B P$ is the fixer regression effect of block position; $R_{1}(H)$ is the random effect of side in the house; $S_{m}(H)$ is the random effect of floor in the house; $E_{n}(H)$ is the random effect of lane in the house; $e_{i j k l m n o}$ is the residual effect.

Then, the adjusted ls-means of the peak area data were used in the association analysis. The association model included the lines (commercial vs. experimental), egg color (WL vs. BL), and breeding value (high vs. low, or regression) as fixed factors. The Kenword-Roger method [20] was used for the adjusted denominator degree of freedom for testing the fixed effects. Generalized linear mixed models (SAS 9.4) were applied to both the adjusted model and the association models. Statistical significance was set at $P \leqslant 0.05$. Coefficients of regression models $(\beta, 95 \% \mathrm{CI})$ were used to determine the effects of metabolites on hatchability traits: HFE, EES, MES, LES and EXP (positive or negative). The statistical model for the commercial line is presented below:

$$
y_{i j k}=\mu+B V_{i}+L_{j}+C_{k}+e_{i j k l}
$$

Where $y_{i j k l}$ is the adjusted ls-means of content of a certain metabolites; $\mu$ is the overall means; $B V_{i}$ is fixed effect of breeding value (group or regression); $L_{j}$ is the fixed effect of line; $C_{k}$ is the fixed effect of egg color; $e_{i j k l}$ is the residual effect.

Next, we investigated metabolite-metabolite associations in the whole data set. An unbiased reconstruction of metabolic networks from this large-scale metabolomics data was built by Gaussian graphical model (GGM) as described previously by Krumsiek [21]. GGM is induced by partial correlation coefficient, which is pairwise Pearson correlation coefficients conditioned against the correlation with all other metabolites. A detailed description of the GGM calculation procedure in metabolic data can be found in[21]. 


\subsection{Results}

\subsubsection{The Identified Metabolites in Egg Yolks}

In total, 105 known and 143 unknown metabolites were profiled in this experiment. Among these known metabolites, 42 types of fatty acids, 22 types of amino acids, 11 types of steroids, 9 types of carbohydrates, 8 types of glycerides, 6 types of vitamins, 2 types of organic acids and 5 types of other group nutrients were involved (Figure 4.2).

Nutrients in the egg yolk whose contents were significantly different in the two hatchability groups of HTE (HBV vs. LBV) were considered to be important metabolites for hatchability. To determine the functions of these important metabolites influencing the embryonic survivability these, we estimated their ls-mean values in the separated breeding value groups (Table 4.1). If the 1s-mean in the high breeding value group (HBV) was higher than the corresponding value in the low breeding value group (LBV), then that metabolite would have a positive effect on HTE; otherwise, it would impede the embryonic survivability. 2 vitamins, 3 steroids, 16 fatty acids and 2 amino acids were significantly related to the hatchability of all of the egg sets in the 4 lines (Table 4.1). The commercial brown line had the largest amount of significant metabolites (10 metabolites), while the commercial white line had 8 metabolites identified, the experimental brown line had 5 metabolites, and the experimental white line had only 1 metabolite (Table 4.1).

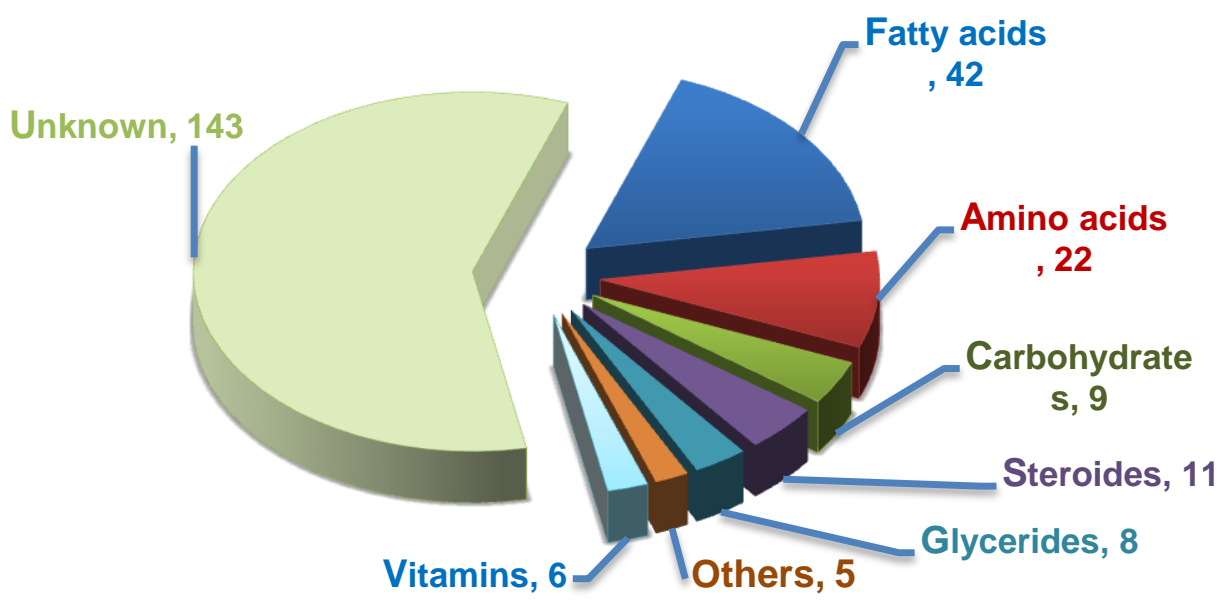

Figure 4.2: Clusters of chicken egg yolk metabolites in the metabolite profile experiment 
It was found that a few metabolites, which were highly related or involved in the same pathways, had totally opposite effect on embryonic development. For example, both squalene and campesterol are precursors for cholesterol generation, but squalene had a positive effect on $\mathrm{HTE}(\mathrm{HBV}, 11.60 \pm 0.03$; LBV, 11.47 \pm 0.03 ) and campesterol had a negative effect (HBV, 8.03 \pm 0.03 ; LBV, 8.13 \pm 0.03 ). Besides, the same metabolite could have diametrically opposite impacts on HTE when it functioned in different lines. For instance, 9-octadecenoic acid in the commercial brown line had a negative effect (HBV, 19.19 \pm 0.02 ; LBV, $19.27 \pm 0.03$ ), but in the commercial white line, a positive effect was noted (HBV, 19.24 \pm 0.02 ; LBV, 19.18 \pm 0.02$)$. Even if that, most of the metabolites only impacted HTE in one line, except for 9-octadecenoic acid.

Table 4.1: The metabolites associated with the hatchability of all egg sets (HTE) in different lines ${ }^{1}$

\begin{tabular}{lllll}
\hline \multirow{2}{*}{ Nutrient in egg yolk } & Line & \multicolumn{2}{l}{ Hatchability groups } & \multirow{2}{*}{$P$-difference } \\
\cline { 4 - 5 } & & HBV & LBV & \\
\hline Vitamin & BLE & $4.76 \pm 0.05$ & $4.59 \pm 0.05$ & $0.026^{*}$ \\
$\quad$-Tocopherol & WLC & $9.75 \pm 0.02$ & $9.83 \pm 0.02$ & $0.003^{* *}$ \\
Pantothenic acid & &
\end{tabular}

Steroid

$\begin{array}{lllll}\text { Squalene } & \text { BLE } & 11.60 \pm 0.03 & 11.47 \pm 0.03 & 0.008^{* *} \\ \beta \text {-Sitosterol } & \text { BLC } & 6.29 \pm 0.03 & 6.40 \pm 0.03 & 0.002^{* *} \\ \text { Campesterol } & \text { BLC } & 8.03 \pm 0.03 & 8.13 \pm 0.03 & 0.006^{* *}\end{array}$

Amino acid

$\begin{array}{llrrr}\text { Valine } & \text { WLC } & 11.59 \pm 0.02 & 11.63 \pm 0.02 & 0.044^{*} \\ \text { Aspartic acid } & \text { BLC } & 12.02 \pm 0.02 & 11.96 \pm 0.02 & 0.049^{*}\end{array}$

Fatty acid

$\begin{array}{lllll}\text { Hexadecanoic acid } & \text { WLC } & 19.15 \pm 0.02 & 19.09 \pm 0.02 & 0.011^{*} \\ \text { Octadecanoic acid } & \text { WLE } & 18.12 \pm 0.04 & 18.00 \pm 0.04 & 0.018^{*} \\ \text { Eicosatetraenoic acid } & \text { WLC } & 15.53 \pm 0.02 & 15.45 \pm 0.02 & 0.009^{* *}\end{array}$


Chapter 4

$\begin{array}{lllll}\text { Docosatetraenoic acid } & \text { BLC } & 16.93 \pm 0.03 & 16.84 \pm 0.03 & 0.040^{*} \\ \text { Docosapentaenoic acid } & \text { BLE } & 10.52 \pm 0.05 & 10.33 \pm 0.05 & 0.014^{*} \\ \text { Docosahexaenoic acid } & \text { WLC } & 14.43 \pm 0.03 & 14.35 \pm 0.03 & 0.026^{*} \\ \text { Octadecadienoic acid } & \text { BLC } & 10.80 \pm 0.01 & 10.84 \pm 0.02 & 0.035^{*} \\ \text { 9-Octadecenoic acid } & \text { BLC } & 19.19 \pm 0.02 & 19.27 \pm 0.03 & 0.033^{*} \\ \text { 9-Octadecenoic acid } & \text { WLC } & 19.24 \pm 0.02 & 19.18 \pm 0.02 & 0.034^{*} \\ \text { 9-Hexadecenoic acid } & \text { WLC } & 13.78 \pm 0.03 & 13.61 \pm 0.03 & <0.001^{* *} \\ \text { 7-Hexadecenoic acid } & \text { WLC } & 14.28 \pm 0.03 & 14.18 \pm 0.03 & 0.013^{*} \\ \text { 10-Heptadecenoic acid } & \text { BLC } & 16.89 \pm 0.02 & 16.83 \pm 0.02 & 0.043^{*} \\ \text { Hexadecanoic acid, 14-methyl } & \text { BLE } & 10.72 \pm 0.10 & 10.40 \pm 0.12 & 0.037^{*} \\ \text { Tridecanoic acid, 12-methyl } & \text { BLE } & 9.84 \pm 0.07 & 10.08 \pm 0.08 & 0.034^{*} \\ \text { Tetradecanoic acid, 13-methyl } & \text { BLC } & 12.95 \pm 0.03 & 12.86 \pm 0.03 & 0.034^{*} \\ \text { Tetradecanoic acid, 12-methyl } & \text { BLC } & 13.13 \pm 0.03 & 13.04 \pm 0.03 & 0.014^{*} \\ \text { Pentadecanoic acid, 14-methyl } & \text { BLC } & 13.08 \pm 0.03 & 12.98 \pm 0.03 & 0.009^{* *}\end{array}$

\footnotetext{
${ }^{1}$ Values are the ls-means \pm SEMs of the adjusted peak area (no unit) from the generalized linear mixed model. The original peak area data from the GC-MS experiment were log-transformed and adjusted according to the p-rep design that was used for the metabolite profiling [19], with blocks corresponding to days. In the adjusted model, the effects that were related to the laboratory, i.e., the block (day) and position of analysis of the sample, were considered random and covariate, respectively. BLC, commercial brown line; WLC, commercial white line; BLE, experimental brown line; WLE, experimental white line; HBV, high breeding value group in the hatchability of all egg sets; and LBV, low breeding value group in the hatchability of all egg sets. $P \leq 0.05$ was considered significant in the association analysis model
}

Some consistency in the fatty acids was still observed. In the case of BCFAs (hexadecanoic acid, 14methyl; pentadecanoic acid, 14-methyl; tetradecanoic acid, 12-methyl; tetradecanoic acid, 13-methyl; and tridecanoic acid, 12-methyl), only the brown lines were impacted, regardless of whether they were commercial or experimental lines (Table 4.1). Tridecanoic acid, 12-methyl appeared to be an inhibitory factor for the experimental brown line embryonic development (HBV, 9.84 \pm 0.07 ; LBV, $10.08 \pm 0.08$ ); in contrast, the other 4 BCFAs positively influenced HTE (HBV $>$ LBV). The MUFAs, mainly 9-octadecenoic acid and 9-hexadecenoic acid, affected the commercial lines but not the experimental lines. All of the PUFAs (docosahexaenoic acid, docosapentaenoic acid, docosatetraenoic 


\section{Chapter 4}

acid, and eicosatetraenoic acid) except for octadecadienoic acid positively stimulated the embryonic survival ability (HBV>LBV).

In the association analysis between metabolites contents and breeding values in HFE, EES, MES, LES and EXP, totally, 24 fatty acids, 4 vitamins, 9 steroids, and 7 amino acids were significantly related to different hatching traits $(P \leqslant 0.05$, Figures 4.3 and 4.4). Results in HFE, EES, MES, LES and EXP analysis were consistent with HTE association analysis results. 5 BCFAs (hexadecanoic acid, 14methyl; pentadecanoic acid, 14-methyl; tetradecanoic acid, 12-methyl; tetradecanoic acid, 13-methyl; and tridecanoic acid, 12-methyl) impacted only the brown line, and except for tridecanoic acid, 12methyl, all of them stimulated the hatchability or embryonic survival ability; additionally, most of the PUFAs and MUFAs had positive effects, though 9-octadecenoic acid, 9-hexadecenoic acid and octadecadienoic acid had opposite effects in distinct lines.

\subsubsection{Metabolic Network Reconstruction}

GGM analysis of the combined egg metabolites data sets in the four strains revealed a modular structure with respect to metabolic classes, as shown in Figure 4.5. Polar compounds, e.g., amino acids, carbohydrates, vitamin sugars, and lipid components, exhibited separation. The branched fatty acids group, monounsaturated fatty group and other subgroup of fatty acids can clearly be identified. The next step in our analysis was the manual investigation of metabolite pairs displaying strong partial correlation coefficients. The top strong partial correlations are listed in Figure 4.7. Notably, palmitic acid (C16:0) and oleic acid (C18:1) were closely correlated and can be transformed into each other through the monounsaturated fatty acid biosynthesis pathway. This relationship might explain the associations of both palmitoleic acid (C16:1) and oleic acid with embryonic development. Similarly, polyunsaturated fatty acids (C22:4 n-6 docosatetraenoic acid and C22:5 n-6 docosapentaenoic) and branched fatty acids (iso-C14:0 tridecanoic acid, 12-methyl and iso-C15:0 tetradecanoic acid, 13methyl) were also separately correlated. 


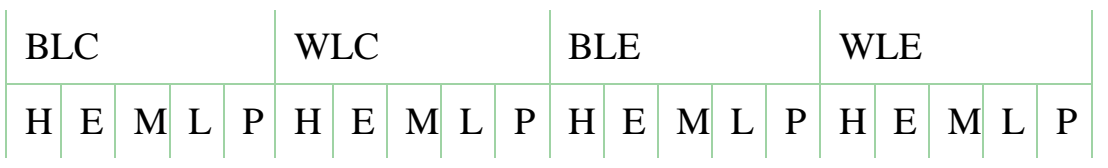

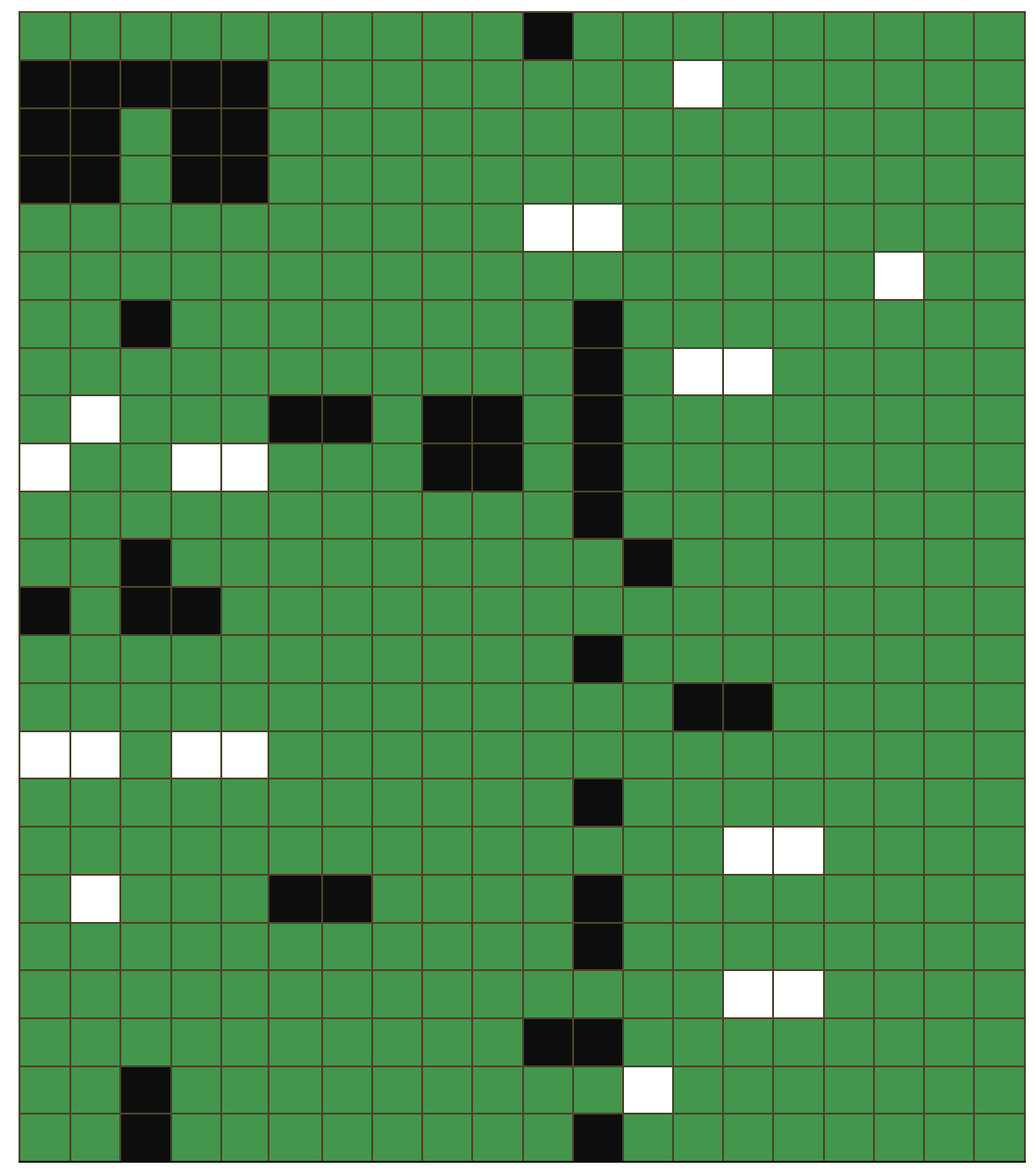

Hexadecanoic acid, 14-methyl Pentadecanoic acid, 14-methyl Tetradecanoic acid, 12-methyl Tetradecanoic acid, 13-methyl Tridecanoic acid, 12-methyl 11-Eicosenoic acid 10-Heptadecenoic acid 7-Hexadecenoic acid 9-Hexadecenoic acid 9-Octadecenoic acid Docosahexaenoic acid Docosapentaenoic acid Docosatetraenoic acid Eicosatetraenoic acid Eicosatrienoic acid Octadecadienoic acid Heptadecanoic acid Hexacosanoic acid Hexadecanoic acid Myristoleic acid Octadecanoic acid Pentadecanoic acid Tetracosanoic acid Tetradecanoic acid

No significant association $(P>0.05)$

Negative significant association $(\beta<0$ and $\mathrm{P} \leqslant 0.05)$

Positive significant association $(\beta>0$ and $\mathrm{P} \leqslant 0.05)$

Figure 4.3: Egg yolk fatty acids associated with the hatchability of fertilized egg sets (HFE), early embryonic survival ability (EES), middle embryonic survival ability (MES), late embryonic survival ability (LES) and external pipping ability (EXP). BLC, commercial brown line; WLC, commercial white line; BLE, experimental brown line; WLE, experimental white line; $\mathrm{H}$, hatchability of the fertilized egg sets; $\mathrm{E}$, early embryonic survival ability; M, middle embryonic survival ability; L, late embryonic survival ability; $\mathrm{P}$, external pipping ability; and $\beta$, coefficient in the regression association model. 


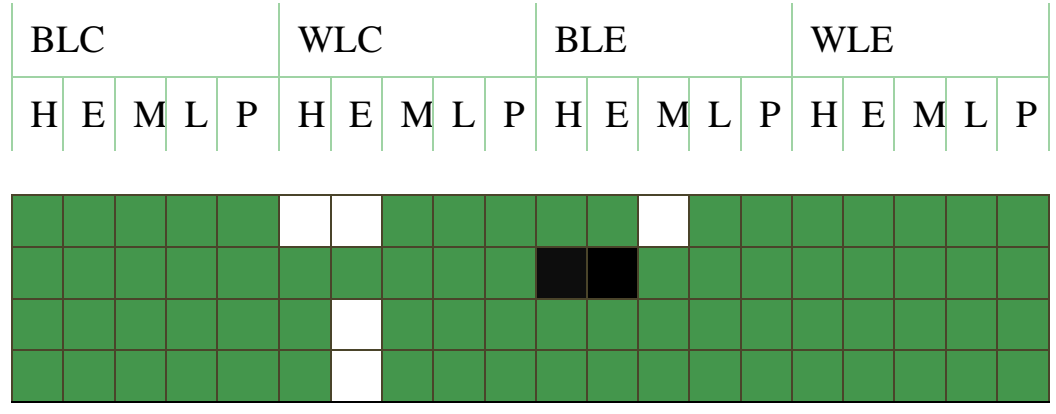

Pantothenic acid

$\delta$-Tocopherol

$\alpha$-Tocopherol

Vitamin

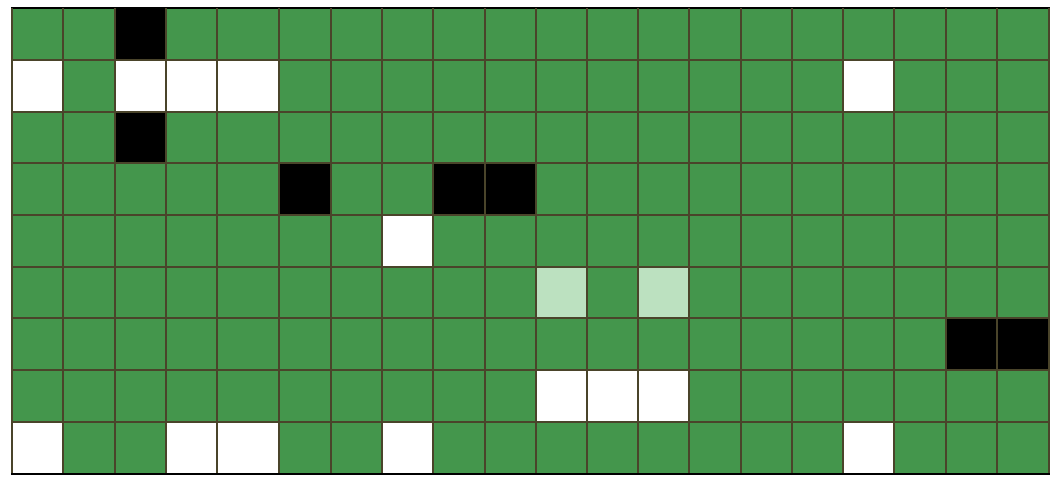

4-Cholesten-3-one

Campesterol

Cholesta-4,6-dien-3-one

Cholesterol

Desmosterol

Steroid

Lanosterol

Lathosterol

Zymosterol

$\beta$-Sitosterol

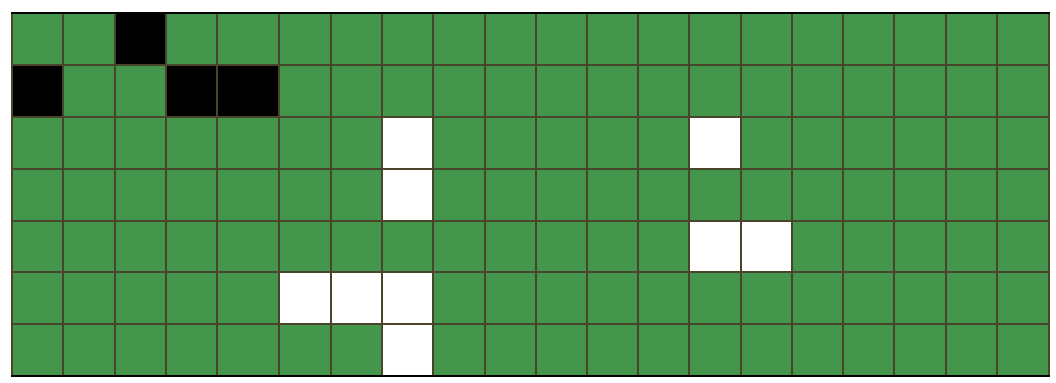

Alanine

Aspartic acid

Citrulline

Leucine

Ornithine-1,5-lactam

Amino

Proline

Valine

acid

No significant association $(P>0.05)$

Negative significant association $(\beta<0$ and $\mathrm{P} \leqslant 0.05)$

Positive significant association $(\beta>0$ and $\mathrm{P} \leqslant 0.05)$

Figure 4.4: Egg yolk vitamins, steroids and amino acids associated with the hatchability of fertilized egg sets (HFE), early embryonic survival ability (EES), middle embryonic survival ability (MES), late embryonic survival ability (LES) and external pipping ability (EXP). BLC, commercial brown line; WLC, commercial white line; BLE, experimental brown line; WLE, experimental white line; $\mathrm{H}$, hatchability of the fertilized egg sets; $\mathrm{E}$, early embryonic survival ability; $\mathrm{M}$, middle embryonic survival ability; L, late embryonic survival ability; $\mathrm{P}$, external pipping ability; and $\beta$, coefficient in the regression association model. 
Chapter 4

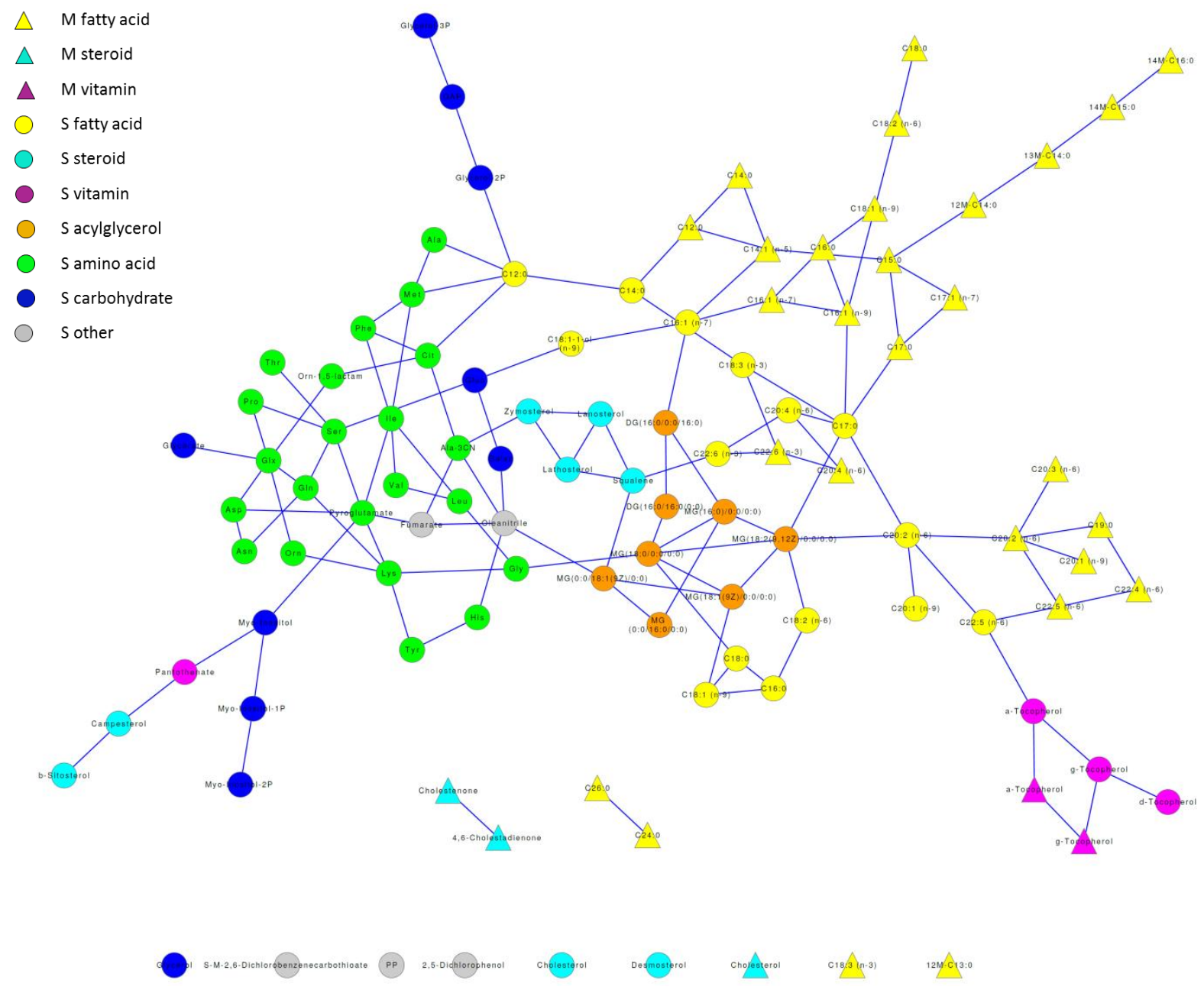

Figure 4.5: Gaussian graphical model inferred from the egg yolk metabolites data set. 
Chapter 4
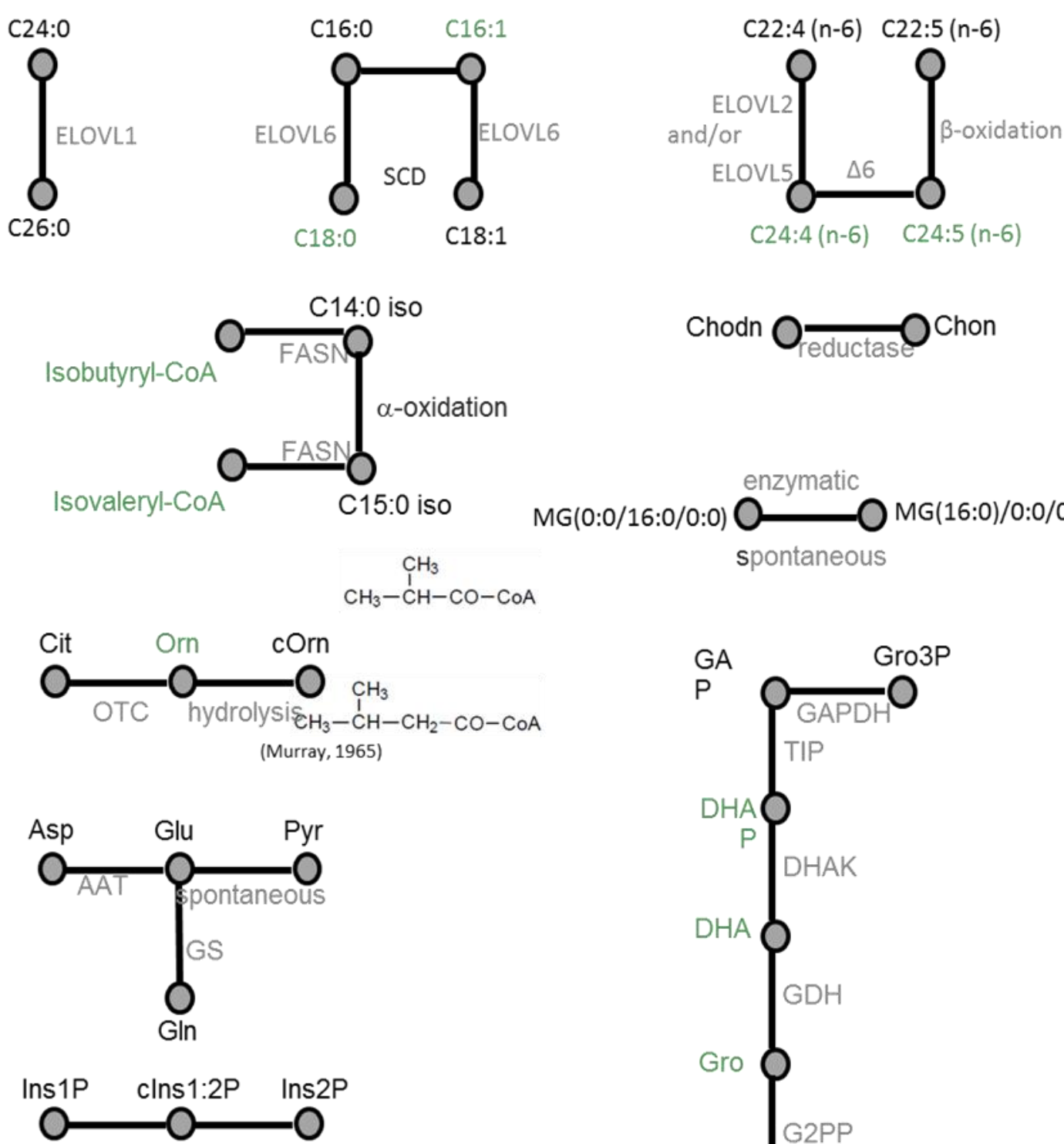

cyclic hydrolase cyclic hydrolase

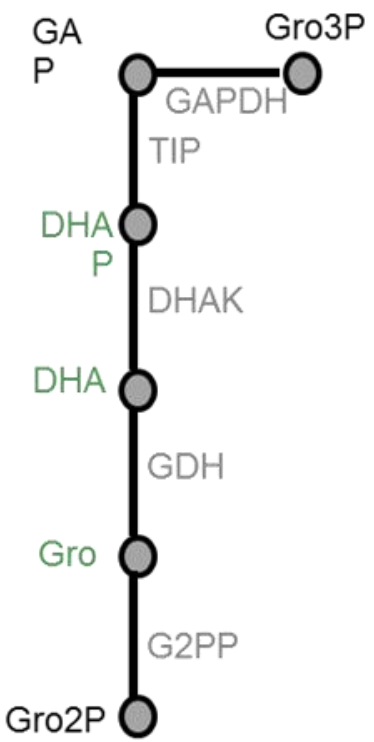

Figure 4.6: Biochemical subnetworks identified between high-scoring metabolite pairs in the GGM network. The pathway connection information was identified based on the KEGG database. 


\subsection{Discussion}

The results illustrate that fatty acids have a greater impact than other egg yolk nutrient clusters on hatchability (24 fatty acids, 4 vitamins, 10 steroids, and 7 amino acids). Subgroups of fatty acids, such as BCFAs, SFAs, MUFAs and PUFAs have been demonstrated correlated with different hatching traits in these 4 lines. As previous research shown, the main components of the yolk are lipids (approximately 65\% of the dry matter) [7]. Proteins and other nutrients compose only approximately $35 \%$ of the yolk [7]. Moran [4] and De Oliveira et al [6] reported that metabolic pathways related to lipids are most active in embryos from mid-incubation until 2 or 3 days before internal pipping. During this phase, adequate oxygen is supplied by the chorioallantois, since glucose availability is low $[4,6]$. Thus, the embryo utilizes yolk fatty acids as the main energy source $[4,6]$. Fatty acids in the egg yolk are stored as triacylglycerol and phospholipids $[4,6]$. During mid-incubation, triacylglycerol is decomposed to free fatty acids and glycerol $[4,6]$. Free fatty acids liberate acetyl-CoA and produce ATP through the $\beta$-oxidation pathway and TCA cycle pathway to guarantee the development of tissues, particularly the liver, heart and pipping muscles $[4,6]$. The remaining glycerol backbone can generate glucose via gluconeogenesis $[4,6]$. Additional blood glucose in embryos is stored in the liver and muscle tissues as polymers called glycogen [4, 6]. Christensen et al [22] demonstrated that glycogen is the vital energy source for embryonic survival during the last phase of incubation. Insufficient energy supplementation in the last days would impact pipping muscle activity and increase embryonic mortality. Fatty acids, mainly unsaturated fatty acids, have functions other than energy sources in embryos, such as stimulating neural system development, improving immunity and regulating cholesterol metabolites. These functions may explain why unsaturated fatty acids represented the largest proportion of fatty acids identified as significantly important for chicken hatchability in this study (6 MUFAs, 6 PUFAs, 5 BCFAs and 7 SFAs).

Furthermore, this study revealed that the importance of egg yolk lipids for hatchability varied among lines, especially BCFAs and MUFAs. The brown line relies on BCFAs more than the other lines. Two MUFAs, 9-octadecenoic acid (oleic acid) and 9-hexadecenoic acid (palmitoleic acid), in the commercial brown line have negative effects on all 5 hatchability traits. However, in the commercial 


\section{Chapter 4}

white line and the experimental brown line, these MUFAs are favorable factors. This diversity among strains might be related to differences in egg yolk composition, gas exchange, thermogenesis, and lipid metabolism due to maternal effect and selection effect [23, 24]. Burghelle-Mayeur et al. [23] reported intensive selection of dwarfism in White Leghorn laying hens results in the incorporation of more dietary lipids into the yolk than normal hens and changes the lipid composition of the egg yolk. Nangsuay et al [24] also demonstrated a selection for laying performance has effect on egg composition and the amount of nutrients in the eggs. In addition, Chiristensen et al. $[25,26]$ reported that selection for egg production is associated with the oxygen consumption of embryos during the middle and late incubation stages. Because aerobic metabolism is vital for embryonic survival in this phase, improved oxygen consumption would benefit embryonic thermogenesis and lipid metabolism [7].

BCFAs are common constituents of the lipids of bacteria, fish oil and ruminant animal oil. Normally, the fatty acyl chain is saturated, and the branch is a methyl group; the most common BCFAs are mono-methyl-branched. Chickens do not have pathways for BCFA de novo generation but can rely on diet absorption and acquisition from bacteria in the intestine. Present study found 5 BCFAs (hexadecanoic acid, 14-methyl; pentadecanoic acid, 14-methyl; tetradecanoic acid, 12-methyl; tetradecanoic acid, 13-methyl; and tridecanoic acid, 12-methyl) impacted the brown line embryonic development, and except for tridecanoic acid, 12-methyl, all of them stimulated the hatchability. However, research on BCFAs for poultry health is not sufficient to elucidate this matter. In humans, it has been demonstrated that BCFAs have anti-cancer activities. Yang et al [27] found that tetradecanoic acid, 13-methyl (13-MTD) purified from a soy fermentation product could induce human cancer cell death-after a 2-h treatment with 13-MTD, they reported that human cancer cells rapidly underwent apoptosis. Wongtangtintharn et al [28] noted that 13-MTD and other BCFAs could inhibit fatty acid synthesis in tumor cells, and they considered that 13-MTD had a close relationship with fatty acid synthase (FAS). We suppose BCFAs in chicken embryo may have similar function on inhibiting specific tumor growth, but it is still not clear right now. In addition, BCFAs have a relationship with newborn gastrointestinal (GI) health in humans. It has been reported that BCFAs are major 
components of normal healthy newborn gastrointestinal tracts [29]. In vernix, BCFAs, most of which are 11-26 carbons in anteiso-configuration, constitute $12 \%$ of the dry weight. However, 16-26 isoconfiguration BCFAs are predominant in meconium [29]. These differences illustrate that active metabolism of BCFAs exists in the fetal GI tract and plays a role in gut colonization. Even though vernix is unique to humans, BCFA might have a similar function in maintaining the stability of gut microbiota in poultry embryos.

MUFAs constitute a large group of unsaturated fatty acids and have a large impact on hatchability. Among them, 9-octadecenoic acid (oleic acid, 18:1, n-9) and 9-hexadecenoic acid (palmitoleic acid, 16:1, n-7) are the most important and compose 40 percent of the total fatty acids in egg yolk [7]. In HTE, EES, LES and EXP of the Lohmann white line and the FLI brown lines, these two fatty acids have positive effects on embryonic development. Similarly, Donaldson and Fites [30] found that increased stearic acid (18:0) and decreased oleic acid in the diet would induce embryonic mortality. Normally, the ratio of 18:0 to 18:1 could not be allowed to exceed 0.25 ; otherwise, hatchability would be adversely affected [31]. In recent study, feeding chicken with conjugated linolenic acid (CLA) would dramatically change the ratios of 16:0/16:1 and 18:0/18:1 in egg yolk and would then interfere with the ratio of SFAs to MUFAs [32]. Aydin et al [32] also mentioned that a high ratio of SFAs to MUFAs could obstruct fatty acid absorption in developing embryos and could subsequently embryonic energy deficiency. Moreover, oleic acid and other long-chain fatty acids are related to cholesterol esterification and low-density lipoprotein (LDL) metabolism. Rumsey et al [33] illustrated that oleic acid and other long-chain fatty acids could first stimulate acyl-CoA: cholesterol acyltransferase (ACAT) activity and then decrease intracellular cholesterol and feedback to stimulate LDL receptor activity. Thus, oleic acid could play a crucial role in the regulation of intracellular cholesterol levels. However, some human studies have indicated that excess intake of oleic acid and other MUFAs will promote insulin resistance, thus increasing the risk of many diseases in humans [34]. This finding could be the reason why oleic acid and palmitoleic acid accompanied by octadecadienoic acid (octadecadienoic acid is close to oleic acid metabolism) in some cases have negative effects on HTE, EES, LES and EXP, in the Lohmann brown line. 


\section{Chapter 4}

Current study shows 5 polyunsaturated fatty acids (docosahexaenoic acid, docosapentaenoic acid, docosatetraenoic acid, eicosatetraenoic acid, and eicosatrienoic acid) promoted hatchability and hatchability traits in specific lines. Among them, docosahexaenoic acid (DHA, 22:6, n-3) is one of the most important PUFAs and is a well-known nutritional factor for neural system development [12]. In avian species, chicken is a special species because it has a precocial hatching mode. Due to pre hatching maturation of brain, newly hatched chicken are able to open eyes, regulate body temperature and rely on themselves to find food. This advantage enables chicken to have strong adaptability in few days after hatching. Similar to DHA, eicosatetraenoic acid (AA, 20:4, n-6) could also stimulate neural development. In addition, AA and eicosapentaenoic acid (EPA, 20:5, n-3, EPA always coexists with DHA in chicken body) are the precursors of eicosanoids, molecules known to stimulate biological activity in platelets, vascular smooth muscle, bronchopulmonary function, gastrointestinal integrity and reproduction [35]. One of the requirements for PUFAs in developing embryonic tissues is to maintain adequate precursors for eicosanoid biosynthesis. Rapid tissue proliferation in mitosis demands that sufficient substrate be available to form cell membranes and organelles. In the case of eicosanoid production, prostaglandin E1 can control cell fusion of chick embryo myoblasts. In addition to the responses observed in myoblasts, prostaglandins E1 and E2 have stimulatory effects on chondrogenesis in vitro. [36]

Campesterol, squalene and sitosterol influenced the embryonic survival ability in HTE; all three molecules can be converted to cholesterol via a steroidogenesis pathway. In chicken, there is a certain level of cholesterol that is required in the embryo, and attempts to lower this level would result in reduced hatchability $[37,38]$. Tocopherol (vitamin E) is an important metabolite for HTE, as shown in results. Vitamin E is primarily known as an antioxidant with respect to reducing cellular free radical damage. In addition, vitamin E can affect the development and maintenance of immunocompetence through multiple functions by acting directly on the immune cell or by indirectly altering metabolic and endocrine parameters, which in turn influence immune function [39]. Vitamin E supplementation in mammals has been shown to increase interleukin (IL)-2 production, which leads to enhanced T cell 


\section{Chapter 4}

proliferation and reduces the production of prostaglandin E, a T-cell suppressive factor, as a result of decreased peroxynitrite formation [40, 41].

The metabolite correlation structures in clearly show the relationships in the metabolic system. Metabolites that belong to the same group or subgroup are much more closely related. Thus, the vitamin, amino acid, fatty acid and other metabolite groups are easily identified. These correlations suggest that metabolites in the same group have similar functions in animal physiological processes, consistent with previous observations that metabolites in the same group, e.g., branched fatty acids, have the same impact on embryonic development. It could be helpful in the detection of crucial pathways for improving embryonic survival ability. Besides, the presence of strong partial correlations in the absence of known metabolic connections could point out missing pathway information or regulatory effects not captured in a simple stoichiometric representation of the pathway. However, identifying the most important or key metabolite in a specific pathway is more difficult. The first metabolite in a pathway may not be the most important in the physiological process for hatchability but still might be identified as significant one in analysis due to its close relationship with other metabolites in this pathway.

\subsection{Abstract}

Chicken egg yolk is the main nutritional source for embryonic development during incubation. However, to date, research on the impact of egg yolk metabolites on hatching has been inadequate. This research is aimed at determining the relationship between egg yolk nutrients and chicken embryonic survival traits and attempts to identify metabolites important for the embryonic health of chickens. Based on an estimation of the hatchability of all egg set (HTE) breeding values, chickens from 4 lines were each classified into high and low hatchability groups and used in the metabolite profile experiments. Generalized linear mixed models were applied in the association analysis of metabolites and hatchability traits (HTE and 5 other traits). We found, in total, 24 fatty acids, 4 vitamins, 10 steroids, and 7 amino acids were significantly related to the hatching traits $(P \leqslant 0.05)$. Fatty acids had the greatest impact on hatchability among these egg yolk nutrient groups. Five branched-chain fatty acids (hexadecanoic acid, 14-methyl; pentadecanoic acid, 14-methyl; 


\section{Chapter 4}

tetradecanoic acid, 12-methyl; tetradecanoic acid, 13-methyl; and tridecanoic acid, 12-methyl) were only associated with hatching traits in the brown lines. Two monounsaturated fatty acids (9hexadecenoic acid and 9-octadecenoic acid) and 9, 12-octadecadienoic acid impeded embryonic survival in the Lohmann brown line but had the opposite effect on the other lines. Five polyunsaturated fatty acids (docosahexaenoic acid, docosapentaenoic acid, docosatetraenoic acid, eicosatetraenoic acid, and eicosatrienoic acid) promoted hatchability and hatchability traits in specific lines. Therefore, certain types of egg yolk nutrients among the amino acids, vitamins, steroids and fatty acids were related to embryonic development in chicken, with fatty acids being the most important. We suggest these identified metabolites may be useful for improving the embryonic health of chickens and other species.

\subsection{References}

1. Wolc A, White IM, Hill WG, Olori VE. Inheritance of hatchability in broiler chickens and its relationship to egg quality traits. Poultry Sci 2010;89:2334-40.

2. Heier BT, Jarp J. An epidemiological study of the hatchability in broiler breeder flocks. Poultry Sci 2001;80:1132-8.

3. Beaumont C, Millet N, Le Bihan-Duval E, Kipi A, Dupuy V. Genetic parameters of survival to the different stages of embryonic death in laying hens. Poultry Sci 1997;76:1193-6.

4. Moran ET. Nutrition of the developing embryo and hatchling. Poultry Sci 2007;86:1043-9.

5. Speake BK, Deans EA. Biosynthesis of oleic, arachidonic and docosahexaenoic acids from their C 18 precursors in the yolk sac membrane of the avian embryo. Comp Biochem Physiol B Biochem Mol Biol 2004;138:407-14.

6. De Oliveira JE, Uni Z, Ferket PR. Important metabolic pathways in poultry embryos prior to hatch. World Poultry Sci J 2008;64:488-99.

7. López-Fandiño R, Anton M, Schade R, eds. Bioactive Egg Compounds. Berlin: Springer Verlag, 2007:33-66.

8. Kuksis A. Yolk lipids. Biochim Biophys Acta 1992;1124:205-22. 
9. Muma E, Palander S, Näsi M, Pfeiffer AM, Keller T, Griinari JM. Modulation of conjugated linoleic acid-induced loss of chicken egg hatchability by dietary soybean oil. Poultry Sci 2006;85:712-20.

10. Golzar Adabi SH, Ahbab M, Fani AR, Hajbabaei A, Ceylan N, Cooper RG. Egg yolk fatty acid profile of avian species-influence on human nutrition. J Anim Physiol Anim Nutr 2013;97:27-38.

11. Darios F, Davletov B. Omega-3 and omega-6 fatty acids stimulate cell membrane expansion by acting on syntaxin 3 . Nature $2006 ; 440: 813-7$.

12. Speake BK . Metobolism and functions of omega-3 fatty acids during development of the avian embryo. In Teale MC, ed. Omega 3 Fatty Acid Research. New York, NY: Nova Science Publishers; 2006:187-223.

13. Kadam MM, Bhanja SK, Mandal AB, Thakur R, Vasan P, Bhattacharyya A, Tyagi JS. Effect of in ovo threonine supplementation on early growth, immunological responses and digestive enzyme activities in broiler chickens. Brit Poultry Sci, 2008;49:736-741.

14. Kratzer FH, Knollman K, Earl L, Buenrostro JL. Availability to chicks of biotin from dried egg products. J Nutr 1988;118:604-608.

15. Vieira SL. Chicken embryo utilization of egg micronutrients. Revista Brasileira de Ciência Avícola 2007;9:1-8.

16. Dikmen BY, Sahan U.Correlations between breeder age, egg cholesterol content, blood cholesterol level and hatchability of broiler breeders.Brit Poultry Sci2007;48:98-103.

17. Fiehn O, Kind T. Metabolite profiling in blood plasma. In Metabolomics. New York: Humana Press. 2007:3-17

18. Lisec J, Schauer N, Kopka J, Willmitzer L, Fernie AR. Gas chromatography mass spectrometrybased metabolite profiling in plants. Nat Protoc 2006;1:387-96.

19. Williams E, Piepho HP, Whitaker D. Augmented p - rep designs. Biom J 2011;53:19-27.

20. Kenward MG, Roger JH. Small sample inference for fixed effects from restricted maximum likelihood. Biometrics 1997;53:983-97.

21. Krumsiek J, Suhre K, Illig T, Adamski J, Theis FJ. Gaussian graphical modeling reconstructs pathway reactions from high-throughput metabolomics data. BMC Syst Biol 2011;5:1. 
22. Christensen VL, Wineland MJ, Fasenko GM, Donaldson WE. Egg storage effects on plasma glucose and supply and demand tissue glycogen concentrations of broiler embryos. Poultry Sci 2001;80:1729-35.

23. Burghelle-Mayeur C, Tixier-Boichard M, Merat P, Demarne Y. De novo lipogenesis and lipolysis activities in normal (Dw) and dwarf (dw) White Leghorn laying hens. Comp Biochem Physiol B Biochem Mol Biol. 1989; 93: 773-779.

24. Nangsuay A, Molenaar R, Meijerhof R, Van den Anker I, Heetkamp MJW, Kemp B, Van den Brand H. Differences in egg nutrient availability, development, and nutrient metabolism of broiler and layer embryos. Poultry Sci 2015;94:415-23.

25. Christensen VL, Donaldson WE. Effect of oxygen and maternal dietary iodine on embryonic carbohydrate metabolism and hatchability of turkey eggs. Poultry Sci 1992;71:747-53.

26. Christensen VL, Donaldson WE, Nestor KE. Effect of supplemental oxygen on blood plasma organic acids within embryos from selected lines of turkeys. Poultry Sci 1999;78:1601-5.

27. Yang Z, Liu S, Chen X, Chen H, Huang M, Zheng J. Induction of apoptotic cell death and in vivo growth inhibition of human cancer cells by a saturated branched-chain fatty acid, 13methyltetradecanoic acid. Cancer Res 2000;60:505-9.

28. Wongtangtintharn S, Oku H, Iwasaki H, TODA T. Effect of branched-chain fatty acids on fatty acid biosynthesis of human breast cancer cells. J Nutr Sci Vitaminol 2004;50:137-43.

29. Ran-Ressler RR, Devapatla S, Lawrence P, Brenna, JT. Branched chain fatty acids are constituents of the normal healthy newborn gastrointestinal tract. Pediatr Res 2008;64:605-9.

30. Donaldson WE, Fites BL. Embryo mortality in quail induced by cyclopropene fatty acids: reduction by maternal diets high in unsaturated fatty acids. J Nutr 1970;100:605-10.

31. Tullett SG. Science and the art of incubation. Poultry Sci 1990;69:1-15.

32. Aydin R, Pariza MW, Cook ME. Olive oil prevents the adverse effects of dietary conjugated linoleic acid on chick hatchability and egg quality. J Nutr 2001;131:800-6.

33. Rumsey SC, Galeano NF, Lipschitz L, Deckelbaum RJ. Oleate and other long chain fatty acids stimulate low density lipoprotein receptor activity by enhancing acyl coenzyme A: cholesterol 


\section{Chapter 4}

acyltransferase activity and altering intracellular regulatory cholesterol pools in cultured cells. $\mathbf{J}$ Biol Chem 1995;270:10008-16.

34. Fukuchi S, Hamaguchi K, Seike M, Himeno K, Sakata T, Yoshimatsu H. Role of fatty acid composition in the development of metabolic disorders in sucrose-induced obese rats. Exp Biol Med (Maywood) 2004;229:486-93.

35. Bautista Ortega J. Polyunsaturated fatty acid metabolism in broiler chickens: effects of maternal diet (Doctoral Dissertation). Corvallis, OR: Oregon State University. 2008.

36. Watkins BA. Importance of essential fatty acids and their derivatives in poultry. J Nutr $1991 ; 121: 1475-85$.

37. Dikmen BY, Sahan U. Correlations between breeder age, egg cholesterol content, blood cholesterol level and hatchability of broiler breeders. Brit Poultry Sci 2007;48:98-103.

38. King ori AM. Review of the factors that influence egg fertility and hatchability in poultry. International Journal of Poultry Science 2011;10:483-92.

39. Gershwin ME, Beach RS, Hurley LS. The potential impact of nutritional factors on immunologic responsiveness. In Nutrition and Immunity. Orlando: Academic Press; 1985:1-7.

40. Meydani SN, Han SN, Wu D. Vitamin E and immune response in the aged: molecular mechanisms and clinical implications. Immunol Rev 2005;205:269-84.

41. Lin YF, Chang SJ. Effect of dietary vitamin E on growth performance and immune response of breeder chickens. Asian Australasian Journal of Animal Science 2006;19:884-9 
Chapter 5

\section{CHAPTER 5: GENERAL DISCUSSION}




\section{General Discussion}

\subsection{General Findings}

The aim of this study was to identify factors contributing to the failure of chicken eggs to hatch, to establish the genetic parameters for different hatching traits and to investigate the effect of egg yolk metabolites on chicken embryonic development. These studies are seen as vitally important to the alleviation of embryonic mortalities during artificial incubation by using an integrated approach.

\subsubsection{Chicken Embryonic Survival Ability and Impact Factors}

In chapter 3, the results illustrated that the majority of not-hatching embryos die in the early and the late stages of incubation which are the most critical phases for embryonic survival. The embryonic mortalities are respectively $10 \%$ in early stage, $3 \%$ in middle stage and $15 \%$ in late stage. Embryonic death rates separately peak at day 5 and day 19 for early and late incubation stages. However, embryonic survival ability at different stages varies among lines. The early embryonic survival rates for fertilized eggs equated to $90.5 \%$ for commercial brown laying hens (BLC), $91.4 \%$ for commercial white laying hens (WLC), $89.3 \%$ for experimental brown laying hens (BLE) and $90.2 \%$ for experimental white laying hens (WLE). Middle survival rates were BLC 97.3\%, WLC 97.2\%, BLE 98.5\% and WLE 95.6\%. The late stage survival rates, which are determined in the present study, were BLC $84.1 \%$, WLC $82.7 \%$, BLE $92.4 \%$ and WLE $85.5 \%$. Besides, the internal pipping rates were BLC 90.4\%, WLC 92.0\%, BLE 96.0\%, and WLE 89.4\%. The external pipping rates were BLC 86.9\%, WLC $90.4 \%$, BLE $93.8 \%$ and WLE $88.2 \%$, respectively.

Embryonic development at different stages has distinct physiological characteristics, and is subject to various external or internal factors. During early stage, embryos are extremely sensitive to their incubation environment, such as the incubation temperature and relative humidity [1]. Other than that, several maternal factors such as genetics, age of hens and time of oviposition also affect embryonic survival ability during this stage of embryonic development, $[1,2]$. In case of the middle stage of incubation, an increased amount of nutrients areabsorbed for embryogenesis. Thus, nutritional deficiencies in hens during egg formation can lead to inadequate nutrient supplement for middle 


\section{Chapter 5}

embryonic development, and increase incidence of embryonic deaths during this interval [3]. The late embryonic developmental stage coincides with the increasing demand for oxygen and a series of physiological events such as initiation of pulmonary ventilation, external pipping, and hatching from the shell $[2,4,5]$. During this stage, glycogen reserved in the liver, heart, skeletal muscles, musculus complexus (pipping muscle) and pectoralis is a major source of energy $[1,4,5]$. An increased incidence of late embryonic mortality just prior to pipping is highly related with insufficient glycogen storage in these crucial tissues. The thyroid hormone has been considered as being an essential role in the synthesis of glycogen and its accumulation in liver, heart and muscle tissues [6]. It is reported that high late embryonic mortality commonly concurs with disorders of embryonic thryoid hormone metabolism [6]. In addition, a large proportion of hatch failure in late stage is caused by abnormal embryonic positions III (head under left wing) [7]. The hatching process is characterized by initial pipping of the shell, followed by a rotational movement in a counter-clockwise direction of the embryo, chipping the shell up to $75 \%[3,8,9]$. From malposition III, the embryo is able to pip both the air cell and the shell, but it is difficult to break out of the shell due to a faulty head position [8].

The selection in pure lines for laying performance may influence embryonic metabolic pathways, and result in a retardation of the growth of organs and muscles involved in the hatching process, which in turn affects the pipping and embryonic survival ability [6]. In our results, compared with unselected experimental lines,selected commercial lines have higher early embryonic survival ability. It is consistent with former studies that selection for high laying performance and low body weight can result in eggs with superior embryonic development at ovipositon and improved survival rates $[1,10]$. Furthermore, in this study, the late embryonic survival ability of selected lines is lower than that of experimental lines, and within these groups, white layers are more affected by late death than brown layers (BLC: $84.1 \%$ vs. BLE: 92.4\%; WLC: $82.7 \%$ vs. WLE: $85.5 \%$ ). When considering differences between late survival rates and internal pipping (DIL), and survival rates and external pipping (DEL), it becomesapparent that more embryos in the experimental line are able to pip internally and externally, whereas the embryos in commercial lines are less able to pip internally and died before having free access to atmospheric oxygen and onset the pulmonary activation. 


\subsubsection{Genetic Parameters of Hatching Traits}

In chapter 3, we applied a generalized linear animal model to estimate genetic parameters of hatching traits. The estimated heritabilities are on a low level and in the usual range for fitness traits [11, 12]. The estimated genetic parameters reflect the genetic contribution of different physiological processes, which act at different stages of embryonic development, and may therefore be considered as distinct traits $[11,13]$. The estimated breeding values for fertility vary between $84.6 \%$ and $98.0 \%$ in all lines, and the values for hatchability of fertile eggs vary between $48.1 \%$ and $98.0 \%$. The most significant differences in magnitude of estimated breeding values for fertility (FER), hatchability of all egg sets (HTE), hatchability of fertilised egg sets (HFE) and late embryonic survival ability (LES) are found in WLC $(23 \%, 39 \%, 34 \%$ and 36\%) and the less significant in BLE $(8 \%, 23 \%, 16 \%$ and $1 \%)$. The low estimated heritability of early embryonic survival ability (EES), middle embryonic survival ability (MES) and late embryonic survival ability (LES) may result in a low selection response during the breeding procedure. However, considering the high reproductive performance and low generation interval in chickens, and the wide range in magnitude of estimated breeding hatchability values, it appears to be feasible to successfully select animals for increased embryonic viability. Furthermore, as expected, the estimated genetic correlation between HTE and HFE, FER, EES, MES, LES, EXP are positive for all breeding lines in this study. Genetic correlations between HTE and HFE are positive and very high for all lines. For BLC, WLC and WLE lines, the estimated genetic correlations between HFE and FER, and between HFE and EESare moderate to high and ranged from 0.32 to 0.71 . For BLE, these correlations are substantially higher (0.89 and 0.93). In contrast, the estimated genetic correlation between HFE and LES, and between HFE and EXP, are high for all lines, ranging from 0.68 upwards. A low to moderate genetic correlation is identified between FER and LES, as well as between FER and $\operatorname{EXP}(0.15$ to 0.56$)$.

In previous studies, the estimation of heritability was carried out by using different statistical approaches. Beaumont et al. [11] pointed out the fact that estimated heritability could vary among different models. The estimated heritabilities based on sire component for fertility (FER), early embryonic survival ability (EES), middle embryonic survival ability (MES), late embryonic survival 


\section{Chapter 5}

ability (LES), and hatchability of fertilised egg sets (HFE) were equal to 0.09, 0.09, 0.07, 0.05 and 0.05, respectively [1]. However, heritabilities of FER, EES, MES, LES, and HFE estimated from the dam model were equal to $0.31,0.25,0.20,0.18$ and 0.15 , respectively [11]. Bennewitz et al. [12] applied a Bayesian threshold model for a genetic evaluation of reproductive traits in White Leghorn layers and found the estimated heritability to range from 0.06 to 0.14 . The reason for the observed substantial variability of genetic parameters, which are estimated from different data sets byusing different statistical models and estimation concepts, remains to be further studied. Furthermore, in our study, based on method of opening eggs, the estimatedheritabilities for FER range from 0.095 to 0.129. However, a higher heritability for fertility (0.16) was noted by Lariviere et al. [13]. Compared with method that is based on candled non-broken eggs, the estimated heritability for FER based on broken eggs could be more accurate due to its elimination of the confounding effect of early embryonic mortality and fertility. In terms of the effect of selection for egg performance on fertility, there were essentially no differences between the different breeding lines, regardless of their selection history. These results demonstrate that selection for high performance and egg quality traits has no effect on genetic variation of fertility, although in other studies a negative genetic correlation was found between egg quality traits and fertility based on candling eggs $[14,15,16]$. Although between the experimental and commercial lines there are significant differences in estimated heritabilities for MES, no major differences are observed in heritabilities for FES and LES. It can be concluded that the selection for hatchability traits, which is applied in the commercial breeding program, resultsin a reduced genetic variation of middle embryonic survival ability.

In literature, estimates of genetic correlations between the stages of embryonic survival ability are not consistent. As per the results of this study, and based on the dam component, Beaumont et al. [11] estimated moderate to high genetic correlation between three stages of embryonic survival ability. These results werealso in agreement with the study of Brah et al. [17], who estimated a strong genetic correlation between early mortality and late mortality, and a moderate relationship between both early mortality and pipping mortality, and between late and pipping mortality. However, Lariviere et al. [13] estimated a favourable positive correlation between both EES and LES (0.34) and between MES and 


\section{Chapter 5}

LES (0.11), and a negative correlation was estimated between EES and MES (-0.78). As highlighted by Lariviere et al. [13], discrepancies between different studies in estimates of genetic parameters could be attributed to breed differences or due to a different definition of mortality stages. Therefore, comparing genetic parameters with other studies is difficult due to different models being applied und differences in the genetics of the chicken population.

\subsubsection{Impact of Egg Yolk Metabolites on Embryonic Development}

The results of chapter 4 illustrated that fatty acids have a greater impact than other egg yolk nutrient clusters on hatchability ( 24 fatty acids, 4 vitamins, 10 steroids, and 7 amino acids). Subgroups of fatty acids, such as BCFAs, SFAs, MUFAs and PUFAs have been demonstrated to be correlated with different hatching traits in these 4 lines. Unsaturated fatty acids represent the largest proportion of fatty acids which are identified as significantly important for chicken hatchability in this study (6 MUFAs, 6 PUFAs, 5 BCFAs and 7 SFAs). Moreover, this study reveals that the importance of egg yolk lipids for hatchability varies among lines, especially BCFAs and MUFAs. The brown line relies on BCFAs more than the other lines. Two MUFAs, 9-octadecenoic acid (oleic acid) and 9hexadecenoic acid (palmitoleic acid), in the commercial brown line have negative effects on all 5 hatchability traits. However, in the commercial white line and the experimental brown line, these MUFAs are favourable factors.

As previous research shown, the main components of the yolk are lipids (approximately $65 \%$ of the dry matter) [18]. Proteins and other nutrients compose only approximately $35 \%$ of the yolk [18]. Moran [3] and De Oliveira et al [4] reported that metabolic pathways related to lipids are most active in embryos from mid-incubation until 2 or 3 days before internal pipping. During this phase, adequate oxygen is supplied by the chorioallantois, but the glucose availability in egg is low $[3,4]$. Thus, the embryo utilizes yolk fatty acids as the main energy source [3, 4]. Insufficient energy supplementation at this stage would impact pipping muscle activity prior to pipping and increase late embryonic mortality. Fatty acids, mainly unsaturated fatty acids, have functions other than energy sources in embryos, such as stimulating neural system development, improving immunity and regulating cholesterol metabolites. MUFAs constitute a large group of unsaturated fatty acids, and among them, 


\section{Chapter 5}

9-octadecenoic acid (oleic acid, 18:1, n-9) and 9-hexadecenoic acid (palmitoleic acid, 16:1, n-7) are the most important and compose 40 percent of the total fatty acids in egg yolk [18]. Donaldson and Fites [19] found that increased stearic acid (18:0) and decreased oleic acid in the diet would induce embryonic mortality. In recent study, feeding chicken with conjugated linolenic acid (CLA) would dramatically change the ratios of 16:0/16:1 and 18:0/18:1 in egg yolk and would then interfere with the ratio of SFAs to MUFAs [20]. Aydin et al [20] also mentioned that a high ratio of SFAs to MUFAs could obstruct fatty acid absorption in developing embryos and could subsequently embryonic energy deficiency. In case of polyunsaturated fatty acids, docosahexaenoic acid (DHA, 22:6, n-3) is one of the most important PUFAs and is a well-known nutritional factor for neural system development [21]. Similar to DHA, eicosatetraenoic acid (AA, 20:4, n-6) could also stimulate neural development. Besides, AA and eicosapentaenoic acid (EPA, 20:5, n-3, EPA always coexists with DHA in chicken body) are the precursors of eicosanoids, molecules known to stimulate biological activity in platelets, vascular smooth muscle, bronchopulmonary function, gastrointestinal integrity and reproduction [22]. One of the requirements for PUFAs in developing embryonic tissues is to maintain adequate precursors for eicosanoid biosynthesis, especially prostaglandin E1 and E2 which can control cell fusion of chick embryo myoblasts. In addition to the responses observed in myoblasts, prostaglandins E1 and E2 have stimulatory effects on chondrogenesis in vitro [23].

The results in chapter 4 demonstrated that the same metabolite might have diverse effects in different lines on chicken embryonic development. Some metabolites are even shown to impair embryonic development, which is contrast with previous researches. Former studies have illustrated that dietary linoleic acid could act as a precursor of the C20-22 PUFAs, particularly for the generation of C20:4 n6 arachidonic acid, stimulating embryonic neural system development [24, 25]. In our study, we found linoleic acid, accompanied with oleic acid and palmitoleic acid, also to have a positive effect onhatching traits in the commercial white line and the experimental brown line, whereas, they had negative effects on hatchability of all egg sets (HTE), early embryonic survival ability (EES), late embryonic survival ability (LES) and external pipping ability (EXP)in the commercial brown line. Since the nutritional requirement for embryonic development varies among these lines, we supposed 


\section{Chapter 5}

that effects of maternal diet, breed and selection may induce changes of egg yolk composition. Burghelle-Mayeur et al. [26] reported that intensive selection for dwarfism in White Leghorn laying hens results in the incorporation of more dietary lipids into the yolk than in normal hens and changes the lipid composition of the egg yolk. Nangsuay et al [27] also demonstrated a selection effect on egg composition and the amount of nutrients in the eggs. In case of maternal effects, Wilson [28] and Tůmová and Gous [29] emphasized that maternal diet and age could interfere egg yolk composition. However, it has not been proven that maternal effects or line selection can change the metabolic system of embryos and then induce specific metabolites to impair embryonic development. We suppose that further, more precise metabolites pathway analyses might provide an explanation of these abnormal effects of metabolites.

In chapter 4 metabolite profile researches, we have found lots of unknown metabolites exist in egg yolk, but do not clarify their identities and relationship with chicken hatchability. The unknown metabolite is a small molecule that can reproducibly be detected and quantified in a metabolomics experiment, but whose chemical identity has not been elucidated yet [30]. Unknowns may constitute previously undocumented small molecules, such as rare xenobiotics or secondary products of metabolism, or they may represent molecules from established pathways which could not be assigned using current libraries of GC-MS fragmentation patterns [31, 32]. A considerable number of highranking hits reported in these biomarker studies represent unknown metabolites. However, given to unclarified chemical identities of unknown metabolites, they are limited to be used as functional biomarkers for further investigations and clinical applications. Approaching the problem from a conceptually different perspective, krumseik (2011) [30] presents a novel functional metabolomics method to predict the identities of unknown metabolites using a systems biological framework. They found that GGMs, which are based on partial correlation coefficients, can identify biochemically related metabolites from high-throughput metabolomics data and genomic data [33, 34]. if an unknown compound displays a similar statistical association with a genetic locus in a GWAS or a known metabolite in a GGM, then this may provide specific information of where it is located in the 


\section{Chapter 5}

metabolic network. Based on this information it may be possible to derive testable hypotheses on the biochemical identity of the unknown metabolites.

\subsection{General Conclusions}

The general findings in this study for genetic parameter estimation of chicken hatchability traits and crucial metabolite detection were summarized below:

1) More than $20 \%$ of embryonic deaths occur in the early and the late stages of incubation, and embryonic mortality peaks at day 5 and day 19, respectively. Embryonic development at different stages has distinct physiological characteristics, and might be subject to various external or internal factors.

2) The estimated heritabilities for hatchability traits were on a low level and in the usual range for fitness traits. Low heritabilities might result in a low selection response in the breeding procedure. However, the wide range in magnitude of estimated breeding values for hatchability traits appears to enable a successful selection of animals for increased embryonic viability.

3) A number of metabolites in egg yolk were identified as crucial factors for hatchability. Fatty acids are the greatest largest cluster among them, especially branched fatty acids (BCFAs), monounsaturated fatty acids (MUFAs) and polyunsaturated fatty acids (PUFAs).

4) The same metabolite might have diverse and even opposite effects on embryonic development among in different chicken lines. Functions of some important metabolites for hatchability are contrast with previous researches.

\subsection{Outlook}

Despite the mentioned limitations of the methods applied in our studies we found substantial evidence supporting the hypothesis that many metabolites in egg yolk have a close relationship with chicken embryonic development. In addition to this, we got accurate estimates of genetic parameters from a substantial set of chicken hatching traits. Based on the conclusions from these studies, we suggest to 


\section{Chapter 5}

develop more differentiated and precise metabolite networks, which would require more and better analyses of metabolites in different stages of embryonic development, in order to better understand the physiological process in the chicken embryonic metabolism and ultimately to use this information to develop better methods for breeding value estimation and selection.

\subsection{References}

1. Christensen VL. Factors associated with early embryonic mortality. World Poultry Sci J 2001;57:359-372.

2. Sharifi AR, Horst P, Simianer H. The effect of naked neck gene and ambient temperature and their interaction on reproductive traits of heavy broiler dams. Poultry Sci 2010;89:1360-1137.

3. Moran ET. Nutrition of the developing embryo and hatchling. Poultry Sci 2007;86:1043-9.

4. De Oliveira JE, Uni Z, Ferket PR. Important metabolic pathways in poultry embryos prior to hatch. World Poultry Sci J 2008;64:488-99.

5. Pulikanti R, Peebles ED, Keirs RW, Bennett LW, Keralapurath MM, and Gerard PD. Pipping muscle and liver metabolic profile changes and relationships in broiler embryos on days 15 and 19 of incubation. Poultry Sci 2010;89:860-865.

6. Christensen VL, Donaldson WE, McMurtry JP. Physiological differences in late embryos from turkey breeders at different ages. Poultry Sci 1996;75:172-178.

7. Ernst RA, Bradley FA, Delany ME, Abbott UK, Craig RM. Egg candling and breakout analysis. ANR Publication 8134, Univ of California 2004.

8. Byerly TC, Olsen MW. Causes of the embryonic malposition head-under-left-wing. Poultry Sci $1934 ; 13: 278-282$

9. Decuypere E, Willemsen H. Physiological control mechanisms during late embryogenesis and during pipping and hatching. Post Graduation Course in Incubation Biology and Management. Univ of Wageningen Holland 2006.

10. Coleman JW, Siegel PB. Selection for body weight at eight weeks of age: Embryonic state at oviposition and its relationship to hatchability. Poultry Sci 1966;45:1008-1011. 
11. Beaumont C, Millet N, Le Bihan-Duval E, Kipi A, Dupuy V. Genetic parameters of survival to the different stages of embryonic death in laying hens. Poult Sci 1997;76:1193-6.

12. Bennewitz J, Morgades O, Preisinger R, Thaller G, Kalm E. Variance component and breeding value estimation for reproductive traits in laying hens using a Bayesian threshold model. Poult. Sci 2007;86:823-828.

13. Larivière JM, Michaux C, Leroy $\mathrm{P}$. Genetic parameters of embryonic viability traits in a traditional chicken breed. Int J Poultry Sci 2009;8:1183-1188.

14. Sewalem A, Johansson K, Carlgren AB, Wilhelmson M, Lillpers K. Are reproductive traits impaired by selection for egg production in hens?. J Anim Breed Genet 1998;115:281-297.

15. Rozempolska-Ruciska I, Zieba G, Łukaszewicz M. Hatchability traits as selection criteria in breeding of laying hens. Arch Geflugelkd 2008;73:263-267.

16. Wolc A, White IM, Hill WG, Olori VE. Inheritance of hatchability in broiler chickens and its relationship to egg quality traits. Poultry Sci 2010;89:2334-40.

17. Brah GS, Sandhu JS, Chaudhary ML. Heritability estimates of components of incubation mortality in White Leghorns. Brit Poultry Sci 1991;32:871.

18. López-Fandiño R, Anton M, Schade R, eds. Bioactive Egg Compounds. Berlin: Springer Verlag, 2007:33-66.

19. Donaldson WE, Fites BL. Embryo mortality in quail induced by cyclopropene fatty acids: reduction by maternal diets high in unsaturated fatty acids. J Nutr 1970;100:605-10.

20. Aydin R, Pariza MW, Cook ME. Olive oil prevents the adverse effects of dietary conjugated linoleic acid on chick hatchability and egg quality. J Nutr 2001;131:800-6.

21. Speake BK . Metobolism and functions of omega-3 fatty acids during development of the avian embryo. In Teale MC, ed. Omega 3 Fatty Acid Research. New York, NY: Nova Science Publishers; 2006:187-223.

22. Bautista Ortega J. Polyunsaturated fatty acid metabolism in broiler chickens: effects of maternal diet (Doctoral Dissertation). Corvallis, OR: Oregon State University. 2008.

23. Watkins BA. Importance of essential fatty acids and their derivatives in poultry. J Nutr $1991 ; 121: 1475-85$. 


\section{Chapter 5}

24. Golzar Adabi SH, Ahbab M, Fani AR, Hajbabaei A, Ceylan N, Cooper RG. Egg yolk fatty acid profile of avian species-influence on human nutrition. J Anim Physiol Anim Nutr 2013;97:27-38.

25. Darios F, Davletov B. Omega-3 and omega-6 fatty acids stimulate cell membrane expansion by acting on syntaxin 3. Nature 2006;440:813-7.

26. Burghelle-Mayeur C, Tixier-Boichard M, Merat P, Demarne Y. De novo lipogenesis and lipolysis activities in normal (Dw) and dwarf (dw) White Leghorn laying hens. Comp Biochem Physiol B Biochem Mol Biol. 1989; 93: 773-779.

27. Nangsuay A, Molenaar R, Meijerhof R, Van den Anker I, Heetkamp MJW, Kemp B, Van den Brand H. Differences in egg nutrient availability, development, and nutrient metabolism of broiler and layer embryos. Poult Sci 2015;94:415-23.

28. Wilson HR. Effects of maternal nutrition on hatchability. Poult Sci 1997;76:134-143.

29. Tůmová E, Gous RM. Interaction of hen production type, age, and temperature on laying pattern and egg quality. Poultry Sci 2012;91:1269-1275.

30. Krumsiek J, Suhre K, Evans AM, Mitchell MW, Mohney RP, Milburn MV, Wägele B, RömischMargl W, Illig T, Adamski J, Gieger C, Theis FJ, Kastenmüller G. Mining the unknown: a systems approach to metabolite identification combining genetic and metabolic information. PLoS Genet 2012;8.

31. Horai H, Arita M, Kanaya S, Nihei Y, Ikeda T. MassBank: a public repository for sharing mass spectral data for life sciences. J Mass Spectrom 2010;45:703-714.

32. Afeefy HY, Liebman JF, Stein SE. NIST Chemistry WebBook, NIST Standard Reference Database Number 69. In: Linstrom PJ, Mallard WG, editors 2011.

33. Krumsiek J, Suhre K, Illig T, Adamski J, Theis FJ. Gaussian graphical modeling reconstructs pathway reactions from high-throughput metabolomics data. BMC Syst Biol 2011;5:21.

34. Mittelstrass K, Ried JS, Yu Z, Krumsiek J, Gieger C, Prehn C, Römisch-Margl W, Polonikov A, Peters A, Theis FJ, Meitinger T, Kronenberg F, Weidinger S, Wichmann HE, Suhre K, WangSattler R, Adamski J, Illig T. Discovery of Sexual Dimorphisms in Metabolic and Genetic Biomarkers. PLoS Genet 2011;7. 
Summary

6. SUMMARY 


\section{Summary}

Hatchability is an important trait for the chicken industry because of its high impact on chicken output. The embryonic survival ability in fertile eggs is greatly influenced by the offspring genotype and the quality of the egg environment, especially egg yolk metabolites. This study is aimed at estimating genetic parameters of hatchability traits and determining the relationship between yolk nutrients and chicken embryonic survival ability in order to identify important metabolites for the embryonic health of chickens and other species.

In chapter 2, the functions of egg yolk metabolites were further discussed. We summarized the literature from the past decades on finding metabolites in egg yolk and albumen that impact embryonic survival ability and on determining the function of different nutritional components during distinct stages of incubation. The function of fatty acids, carbohydrates, amino acids, vitamins and steroids were introduced in detail in this chapter. In general, fatty acids related pathways are most active during middle and late incubation stages and associated with chicken embryonic energy metabolism, neural system development, immune ability and lipid uptake. Carbohydrates are an important energy source when embryonic oxygen consumption is insufficient. Essential amino acids control protein synthesis and impact chicken embryonic growth rate. Vitamins with strong anti-oxidation ability could inhibit toxic effect of avidin in the egg. Steroids such as cholesterol are important hormone precursors. A good nutritional status of the parent birds is crucial for the transfer of an adequate, balanced supply of nutrients required to the egg for a normal development of the embryo. The consequences to the embryo may be lethal if the egg contains inadequate, excessive, or imbalanced levels of nutrients.

In chapter 3, we estimated the genetic parameters of hatchability traits: Fertility (FER) hatchability of total eggs set (HTE) and fertilized eggs (HFE) and susceptibilities to early (EES), middle (MES), late (LES) embryonic survival ability, external pipping ability (EXP) and internal pipping ability (INP). 5,983 hatching egg records obtained from 100 unselected Brown layer hens (BL) and 99 White layer hens (WL) from Friedrich Loeffler Institute and 80,916 egg records selected from 2909 Brown layer hens (BLC) and 3319 white laying hens (WLC) from Lohmann company were included in this study. 


\section{Summary}

To determine the time of embryonic mortality, eggs not exhibiting a viable embryo at candling on days 7 and 18 of incubation and eggs that did not hatch were opened and examined macroscopically to assess their true fertility and estimate the time of embryonic death. Heritabilities were estimated using a generalized linear animal model and results suggest that heritability for fertility (FER), hatchability of total eggs (HTE), hatchability of fertilised eggs (HFE), early embryonic survival ability (EES), middle embryonic survival ability (MES), late embryonic survival ability (LES), external pipping

ability (EXP) and internal pipping ability (INP) exhibit ranges of 0.10-0.13, 0.04-0.08, 0.05-0.06, $0.03-0.10,0.01-0.18,0.02-0.09,0.001-0-083$ and $0.001-0.11$, respectively. The estimated genetic correlations between different reproductive traits for all breeding lines were positive ranging from 0.15 to 0.94 . The ranges between minimum and maximum breeding values of HTE and HFE were large and on average approximately $30 \%$. The low heritabilities found for these traits led to the conclusion that the potential selection response is expected to be limited. Taking into consideration the very high reproductive ability and the short generation interval in chicken, it appears still feasible to select animals for improved hatchability.

Based on an estimation of the hatchability of all egg set (HTE) breeding values from the results in chapter 3, chicken from 4 lines were divided into high and low hatchability groups and used to determine the metabolite profile in chapter 4. Generalized linear mixed models were applied in association analysis between metabolites and hatchability traits (HTE and 5 other traits). We found, in total, 24 fatty acids, 4 vitamins, 10 steroids, and 7 amino acids to be significantly related to the hatching traits $(\mathrm{P} \leqslant 0.05)$. Fatty acids usually had the greatest impact on hatchability among these egg yolk nutrient groups. Four branched-chain fatty acids (hexadecanoic acid, 14-methyl; pentadecanoic acid, 14-methyl; tetradecanoic acid, 12-methyl; and tetradecanoic acid, 13-methyl) were associated with hatching traits in the brown lines. Two monounsaturated fatty acids (9-hexadecenoic acid and 9octadecenoic acid) and 9, 12-octadecadienoic acid impeded embryonic survival in the Lohmann brown line but had the opposite effect in the other lines. Five polyunsaturated fatty acids (docosahexaenoic acid, docosapentaenoic acid, docosatetraenoic acid, eicosatetraenoic acid; and eicosatrienoic acid) promoted hatchability and hatchability traits in specific lines. 


\section{Summary}

In conclusion, genetic parameter estimation results suggest that heritabilities for FER, HTE, HFE, EES, MES, LES, EXP and INP are generally low. These results seem to lead to the expectation that the potential selection response will be limited. However, considering the high reproductive performance and low generation interval in chickens and the wide range of estimated breeding values for hatchability, successfully selecting animals for increased embryonic survival appears to be a feasible endeavour. Certain types of egg yolk nutrients among the amino acids, vitamins, steroids and fatty acids were related to embryonic development in chicken, with fatty acids being the most important ones. These identified metabolites may be useful for improving the embryonic health of chickens and other species. 


\section{Acknowledgements}

First, I would like to thank Prof. Dr. Henner Simianer and Prof. Dr. Christoph Knorr for being my supervisors and for their guidance during my PhD study. Especially I express gratitude to Prof. Dr. Henner Simianer, for his valuable advice, great support, patience and encouragement during this time whenever I faced problem on my study and research. All this would have been not possible, if I was not involved in the working group of Prof. Dr. Henner Simianer. I would like to thank you for providing me a good opportunity to study in Germany and let me learn a lot of advanced knowledge in animal science.

I would like to thank Dr. Reza Sharifi for the great collaboration and support during the whole project. I want to thank you for always kindly help me in my data analysis, paper correction and provide me many valuable advices for my questions and concerns during my PhD study.

I also would like to thank Prof. Dr. Jürgen Hummel for being my third supervisor and for giving me the great help in my $\mathrm{PhD}$ work.

I also acknowledge the project support from German Federal Ministry of Education and Research (BMBF) within the AgroClustEr "Synbreed - Synergistic plant and animal breeding" (Grant ID: 0315528C) and a grant from China Scholarship Council, which made this research possible.

I would like thank all my co-authors in genetic parameter estimation and metabolite association works. Thanks to Dr. Steffen Weigend, Prof. Dr. Henner Simianer and Dr. Reza Sharifi for the help in the work on 'metabolites in egg yolk important for poultry development during the incubation period'; Thanks to Dr. Steffen Weigend, Annett Weigend, Prof. Dr. Rudolf Preisinger, Dr. Matthias Schmutz, Prof. Dr. Henner Simianer and Dr. Reza Sharifi for the help in the work on 'statistical and genetic analysis of hatchability traits in laying hens'; Thanks to Dr. Steffen Weigend, Annett Weigend, Prof. Dr. Rudolf Preisinger, Dr. Matthias Schmutz, Dr. Lilla Römisch-Margl, Dr. Ralf Jonczyk, Prof. Dr. Hans-Peter Piepho, Dr. Waqas A Malik, Prof. Dr. Henner Simianer and Dr. Reza Sharifi for the help in the work on 'metabolite profile of chicken egg yolk and its association with hatchability'. 
I am also grateful to all my colleagues from the Animal Breeding and Genetics Group. Thank you for bearing my temperament, for cheering me up whenever I felt down and for your friendship.

Last but not least, I would like to thank my whole family for your unconditional love and support, which gave me the strength to pull through to the end. 


\section{Curriculum Vitae}

Name: $\quad$ Yi Zhang

Date of birth: $\quad$ September 16,1988

Place of birth: $\quad$ Sichuan/China

Nationality: $\quad$ China

\section{Education}

Since $10 / 2012$

Ph.D Student in Agricultural Sciences at Georg-August University,

\section{Göttingen, Germany}

Thesis title: "Studies on Chicken Hatchability Traits and Its Relation with Egg Yolk Metabolites"

Thesis advisor: Prof. Dr. Henner Simianer

09/2010 - 06/2012 Master of Science with Agricultural Sciences at China Agricultural University, Beijing, China

Master's thesis: "Studies on Chicken Breeding for Resistance to Avian Infectious Bronchitis"

Thesis advisor: Prof. Dr. Zhengxing Lian

09/2006 - 06/2010 Bachelor of Science with Agricultural Sciences at Sichuan Agricultural University, Ya'an, China

Bachelor's thesis: “In-ovo Feeding of Nutrient to ducks Influences Posthatch Growth Rate" 
Thesis advisor: Prof. Dr. Liang Li

\section{Professional Experience}

Since 10/2012

$09 / 2010-06 / 2012$

Project: National Science and Technology Major Project on Animal

Trans-genetic Research

Scientific researcher at China Agricultural University, Prof. Dr. Zhengxing

Lian

Project: Association Analysis between Egg Yolk Metabolites and Hatchability in Chicken

Scientific researcher at Georg-August University Göttingen, Prof. Dr.

Henner Simianer 